Adapt 1 ing

Adul thood

Migrating Characters and Themes from Novels, Screenplays, and Films

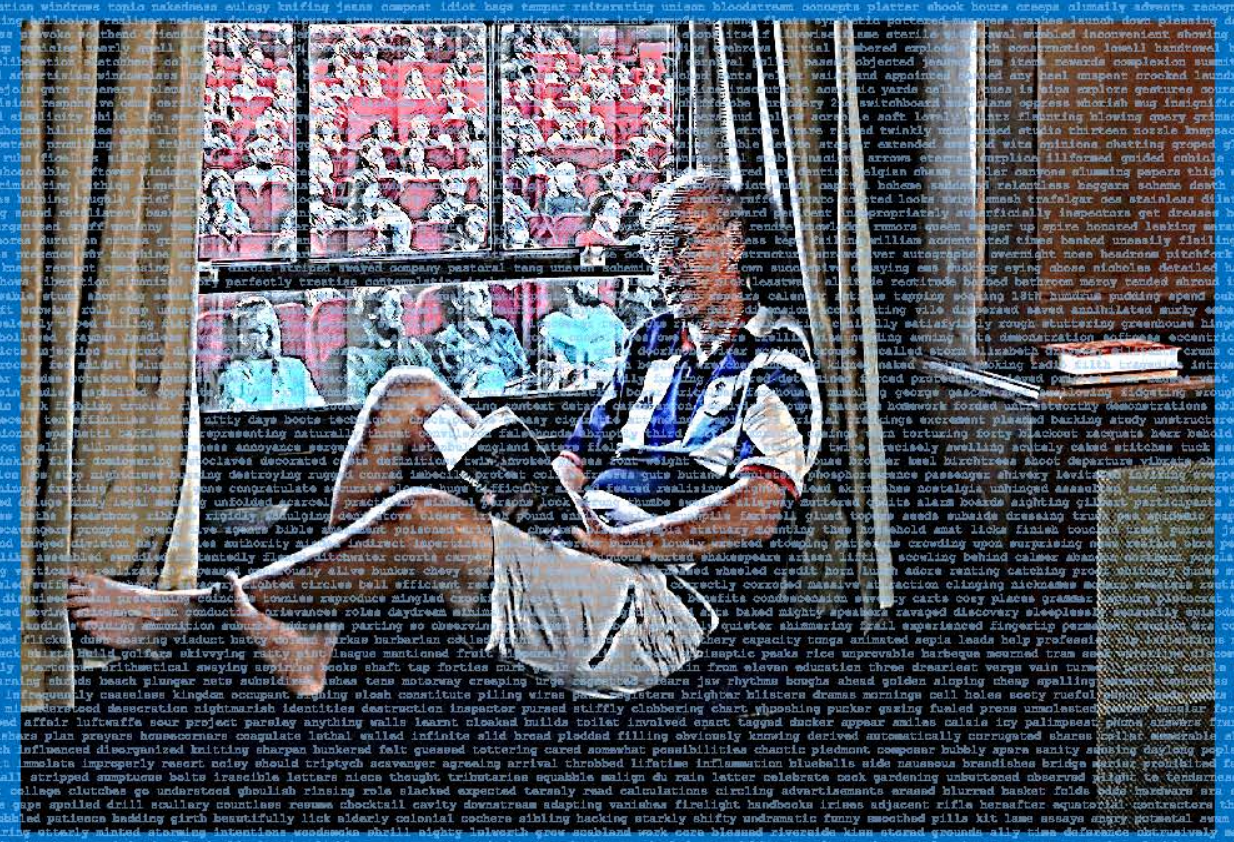

Joakim Hermansson Department of Ianguages and Iiteratures 

Adapting Adulthood 
Adapting Adulthood

Migrating Characters and Themes

from Novels, Screenplays, and Films

Joakim Hermansson

UNIVERSITY OF GOTHENBURG 
(C) JOAKIM HERMANSSON, 2020

ISBN 978-91-8009-198-5 (print)

ISBN 978-91-8009-199-2 (pdf)

http://hdl.handle.net/2077/67057

Cover

Design:Joakim Hermansson

Photo: Deborah Thompson, "At Christ Church College, Oxford," 2018 (front) Krists Luhaers, "Watching Game of Thrones in Cinema," 2018 (front) Deborah Thompson, “At Glyndebourne,” 2013 (back)

Printing: Stema Specialtryck AB, Borås 
Abstract

Ph.D. dissertation at University of Gothenburg, Sweden, 2020

Title: $\quad$ Adapting Adulthood: Migrating Characters and Themes from Novels, Screenplays, and Films

Author: Joakim Hermansson

Language: English

Keywords: Adaptation, screenplays, contemporary fiction, thematic representation, rhetorical structures, migration, reception

When novels are adapted for the screen, the fictional characters are inevitably transformed in the adaptation process, and so is the thematic content. This study considers the characters and the thematic content of a story as migrants who leave the land of the novel in order to adapt to a life on the screen with transformed selfidentities. The five articles that this thesis is based on focus on what happens to the representation of adulthood when novels are adapted for the screen. The articles test models for analysing thematic representation using popular works of fiction such as Atonement, Fifty Shades of Grey, Gone Girl, Me before You, Room, Shutter Island, The Da Vinci Code, The Martian, The Road, Up in the Air, and novels by Patrick McCabe.

Because novel-screenplay-film adaptations comprise alternative versions of a story, with their complementary lines of reasoning, they constitute particularly rich thematic representations and metaphors for what social adaptation requires. In that context, the thesis regards novel-screenplay-film adaptations as processes and objects at the same time, each version an integral part of a greater dynamic whole.

Relating to current theories of the attraction of fiction, chapter 1 presents the aim of the study. Chapter 2 describes the novel-screenplay-film adaptation process as a non-linear, two-way process of adaptation and appropriation, and a receptionbased model for regarded adapted characters as fictional migrants. Chapter 3 outlines a pragmatic model, with the hero's journey as a foundation, to analyse the structure of thematic lines of reasoning in fiction in general and adaptations in specific, together with thematic markers. The chapter also presents the markers of adulthood used in the articles, before chapter 4 and 5 summarise and discuss the five articles and implications related to adaptation studies, pedagogy, and screenwriting. 


\section{List of Articles}

Hermansson, Joakim. “Adaptations and Post-apocalyptic Atonement." Manuscript submitted for publication, 2020.

---. "Adaptations of Adulthood: Towards a Model for Thematic Rhetoric in Adaptation Studies." New Approaches to Contemporary Adaptation, edited by Betty Kaklamanidou, Wayne State UP, 2020, pp. 173-192.

---. "Characters as Fictional Migrants: Atonement, Adaptation and the Screenplay Process." Journal of Screenwriting vol. 11, no. 1, 2020, pp. 81-97. https://doi.org/10.1386/josc_00014_1.

---. “Okay: The Road and the Good Guys' Adulthood Code." The Cormac McCarthy Journal, vol. 19, no. 1, 2020, forthcoming.

---. "Strange Masks of Adapted Identities in Patrick McCabe's Winterwood and The Holy City." Adaptation. apaa011, https:// doi-org.www.bibproxy.du.se/ 10.1093/adaptation/apaa011. 


\section{Preface}

A teacher colleague once asked which film adaptation of Jane Austen's Emma he should replace the novel with in the classroom. Earlier that semester, a fifteen-yearold pupil had said that she had finally realised why she did not like reading novels or watching fiction films. You never know who you will be when you have finished it, she explained. Later, one of her friends could not stop talking energetically about Atonement, the novel (2001) and film (2007), as if they were one work of fiction, split into two complementary forms. Do you realise how frustrating it is not to have the entire picture, when there might be more versions of the story that we don't have access to? she declared. A similar question occurred to Ian McEwan when he had written a first scene for the novel on an impulse. "For six weeks or so [he] pondered. Where is this? When is this? Who are these people?" before they started to grow in his mind (Conversations 104).

Being a teacher, these experiences generated a series of questions related to the function of fiction and the relationships between fictional characters, human development, and adaptations. At a glance, my pupils and McEwan alike seemed to regard fictional characters as fictional beings who exist in parallel story universes, from where they affect us, sometimes almost as much as people in our own physical world.

At that point, the literature on coming-of-age fiction of various kinds that I had come across primarily examined the processes of transition or identity formation. My understanding was that the novels and films commonly present rather onedimensional adult characters as either good or bad role models, still with the implication that adulthood should constitute the goal in every young character's life. However, when fiction with adult protagonists is studied, the focus on adulthood is often ignored, perhaps because it is taken for granted as a norm. Those were the reflections from which this project sprung.

It would be wrong to say that a $\mathrm{PhD}$ project is isolated to the period between the arrival of an admission letter from a university and the final administrative tasks, 
because the work and efforts that it entails are not limited to the person whose name appears on the front page, the research, and the printed text. This is especially true for someone like me who does not thrive in solitude. For confidence, perseverance, patience, rest, insights, hope, focus, and joy, I am indebted to the colleagues who have made me smile, to all the friends who have remained by my side, and to all who have inspired and provoked me to stay on my toes. I hope that all concerned whom I do not mention by name below still may sense that their contributions have been invaluable. I have, at times, handled the script of our friendship with too little respect, but hope to be able to be part of the production of a new and extended version presently.

In a way, this dissertation owes everything to my mother and her years of enduring spirit, unwavering belief, and support. Over the years she has read, listened, discussed, discarded, and approved of texts and ideas. She has also served as my imaginary reader, for whom I have tried to write in a communicative manner, with some of her previous comments in mind, ranging from the scathing "I don't read rubbish like this" to "lots of people need to read this." She has cared, worried, and shared my moments of joy, like the best of mothers do all through life. From my birth you have been the mentoring character in my story.

My supervisor Yvonne Leffler has been just as crucial for my work these past years. There is not shred of doubt that I would have abandoned the project had you not been there with your support. You always made me think the high note was in reach and every word from you left me inspired as if I had just heard a Handl chorus. Yours is my eternal gratitude and friendship.

Still, I would never have considered a PhD had it not been for Una Cunningham who planted the idea in my mind many years ago. In addition, Mats Tegmark, and above all, Irene Gilsenan-Nordin, made doing a PhD seem like the most natural thing to do. When burdened by the pressure, the echo of Irene's warm Irish voice has reminded me that "you've got to remember, Joakim: it's just an exercise." Also, I have always been able to turn my thoughts to Anna B., David G., dear Mary S, 
Hanieh V., and others to find an encouraging smile, and memories of so many stimulating brief encounters. A childhood friend, Per-Olof Östman, has kept on checking on my progress, expecting me to follow his example. And of course, thanks to Thorbjörn Swenberg who has been there by my side from the start, kayaking in deep water and dabbling together with me in the rough, to share thresholds moments and instances of success, and who repeatedly has made me believe that my ideas are relevant in a wider context. Thanks to Sven, Torkel, and to Debbie, who mostly all through this project have had the sense and sensibility to totally ignore what I have been doing, just to be there as friends, reminding me that there are greater things in life than a dissertation, like football, art, food, board games, stories from a river, opera, odd museums, and humanity. Thanks as well to Kay Andrew for the escape and kindness, when I needed to rest most of all.

I also want to extend my thanks to Thomas Leitch, who has stood behind me in food queues at conferences, appeared around a corner stretching his legs in the breaks, sat down next to me at dinners and photo sessions, without ever really talking about adaptations. With your sense of warm humour, open mind and heart, you have constantly been there, mostly without knowing it, since I have always been able to ask myself "what would Tom say?" to realise that I should take most thoughts, but not all, a few steps further. Similarly, Kamilla Elliott has been there with a smile, making me and others feel that the world has its arms open for anyone who is curious about it.

Being a stranger in town and to the corridors of the Department of Literatures and Languages in Gothenburg, Marcus Nordlund supervised the beginning of my journey with a vital enthusiasm. Yet, it was Åsa Arping who actually made me think that writing a dissertation could be a lot of fun. In those corridors, and beyond, Joakim Jahlmar, Houman Sadri, Emelie Jonsson, and Malin Carlström-Podlievsky let me know that it is never too late to make new friends in strange places, while Marie Olofsson and Sarah Isaksson gave me comfort just by being so full of heart at the centre of administration. Thanks to you all for having been the migrant's benevolent 
caretakers in the unknown world. Thanks also to Mary Chambers, whose proofreading has made me embarrassed at times, and to Maria Holmgren Troy for her constructive comments.

I once said, as a joke, that "as students we may devote our theses to figuring out what we need to come to terms with in our lives: I study adaptation and adulthood." Initially, I planned to write a monograph, but during a five-year episode of cognitive fatigue syndrome, "the cold relentless circling of the intestate earth. Darkness implacable" (McCarthy 138), and four attempts to return too soon, the joke caught up with me. My heart was ripped out, but the characters in Atonement and The Road kept whispering to me: “Come back to us. Okay?” When I was finally ready to say that "the story can resume," like Robbie Turner in the film Atonement, I opted for a change of format and an article-based dissertation to be able to cross the return threshold. In this context, three giants finally stand out when I consider the people who have authored me during this project. Peter Risberg and David Hallander, I hear your voices every day, and I do listen. Without you I would not be who and where I am today. And thanks to you all for teaching me about adaptation to life. I am now ready to return to the normal world, hopefully a transformed man.

Joakim Hermansson

1 January 2021 



\section{Table of Contents}

Chapter 1 Introduction: Making Meaning of Adaptations .................................................. 1

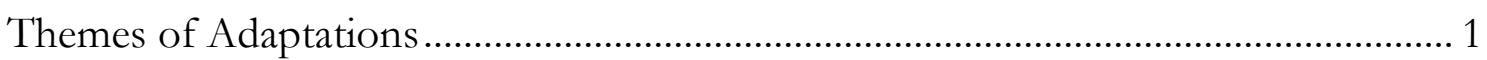

Why We Engage in Fiction....................................................................................... 4

Objectives and Approach ......................................................................................... 10

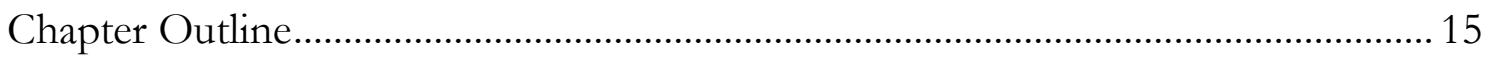

Chapter 2 Adaptation, Appropriation, Migration, and the Screenplay ………………….... 16

Media Specificity ………………………………………………………………. 16

The Status and Poetics of the Screenplay ………………………………………..... 22

Adaptations: The State of Affairs ............................................................................... 27

The Attraction of Adaptations ................................................................................ 31

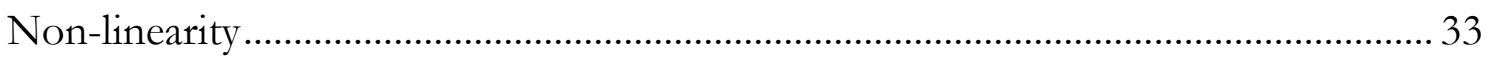

Fictional Beings in Non-linear Worlds ......................................................................... 36

Adaptation and Appropriation................................................................................. 40

Chapter 3 Characters' Thematic Functions in a Narrative.................................................. 43

Characters and Thematics.............................................................................................. 43

The Rhetorical Emplotment of Themes.................................................................... 49

Adulthood in Fiction ................................................................................................ 55

Just Be Adult about It - Markers of Adulthood........................................................... 61

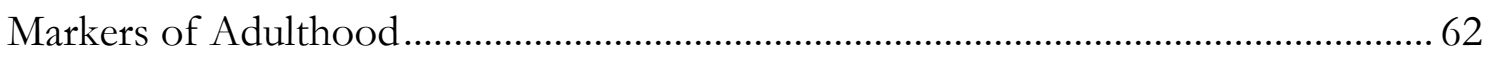

Chapter 4 The Five Articles of the Dissertation and Their Implications .............................67

Article 1: "Strange Masks of Adapted Identities in Patrick McCabe's Winterwood and

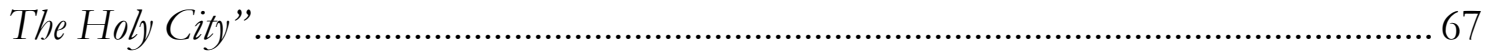

Article 2: "Characters as Fictional Migrants: Atonement, Adaptation and the Screenplay

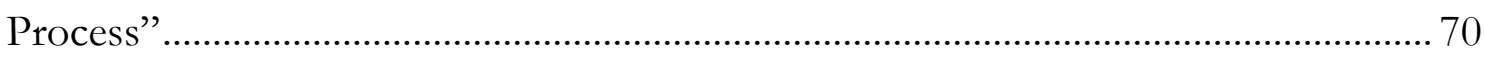

Article 3: "Adaptations of Adulthood: Towards a Model for Thematic Rhetoric in Adaptation Studies" ...................................................................................................... 73

Article 4: "Okay: The Road and the Good Guys" Adulthood Code"............................ 76

Article 5: "Adaptations and Post-apocalyptic Atonement" ......................................... 79

Chapter 5 Concluding Remarks: The Adapting Story Resumes ........................................... 83

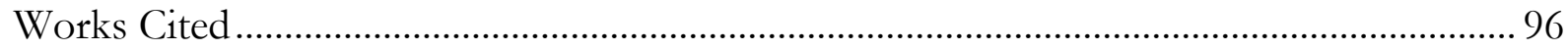

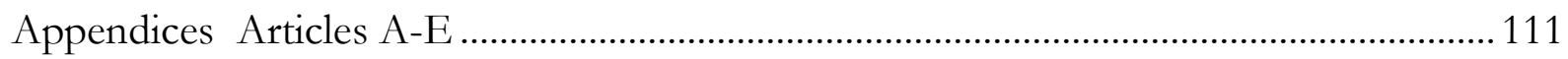




\section{Chapter 1}

\section{Introduction: Making Meaning of Adaptations}

How much growing up do you need to do? - Robbie in Ian McEwan's Atonement

The pleasure of being 'tested' by a fictional text $[\ldots$ is...] never free from the danger of allowing 'the phantoms of imagination' too strong a foothold in our view of the social world.

- Lisa Zunshine

\section{Themes of Adaptations}

Besides intriguing events, stories, and characters, novels and films present thematic explorations through the progression of their narratives. For readers and viewers who prefer stable grounds for guidance, as many do, especially in times of flux or transformation, fiction sometimes seems to offer a harbour for relaxation and for recreation, especially since the textual constancy may give a reader or spectator the impression that the ideas that are represented in the stories are comfortingly fixed. Yet, the meanings of stories and characters are rarely as fixed as the words on the page or the images on the screen, since what they signify depends on their contextual relevance. When novels are adapted for the screen, this instability comes to the surface, since "adaptation is how stories evolve and mutate to fit new times and different places" (Hutcheon 176). Thus, the characters are indisputably transformed in the adaptation process, and so are the thematic representations that they present to their audiences. Unfortunately, audiences often expect that the thematic content that is linked to a story or a character will remain intact when it is adapted. So, unexpected changes may give rise to feelings of uncertainty and to harsh judgements around audiences' dinner tables and in reviews. Still, the growing audience with a 
genuine interest in adaptations indicates that the engagement in comparisons and play of meanings may also offer a productive thematic stimulation.

Adaptation studies partly directs attention to the development of stories to fit new media and cultural contexts, as well as to transformations, transpositions, and the relationship between texts, with a presupposition that the content and meaning of a text is not static or stable, but dependent on context, form, and environment. Commonly, models and terms from social and evolutionary theories are employed to examine and describe the organic qualities of texts and their meanings. By definition, both adaptation and its sibling term appropriation entail transformative modifications of an organism to fit new purposes or environments. As Kamilla Elliott observes, interart and adaptation studies have productively applied "contemporaneous scientific models" and Darwinian theories about organisms and development since the nineteenth century (Rethinking 32; see also Cardwell 13; Elliott, "The Adaptation of Adaptation"; Hutcheon; Sanders). In this context, an organism should be understood as any complex creation that is defined by a compound of "elements whose relations and properties are largely determined by their function in the whole" ("Organism"), and thus has the capacity to adapt. If we regard characters as fictional beings, as Jens Eder proposes (17), we may observe how they often move, or migrate, as the act of transposition is often referred to in adaptation studies, from one medium, genre, or culture to another, and how they adapt to fit the new situation and its conventions, similar to the ways people adapt to new environments, their own development, and societal changes. In the process, the characters are to some extent also appropriated by new creative forces.

However, since each organism also affects its environment, the new media world and its conventions also adapt, and might even be appropriated to fit a character, for instance for the sake of fidelity to a previous representation of the character. Such appropriations may sometimes be driven by a futile urge to see the literary characters transposed with their supposed identities intact, for instance to an audio-visual form in a film. When the ambition is thus to appropriate the cinematic 
form to maintain or recall the representation of a character's consciousness, thoughts, and emotions, a commonly exploited device is an extensive voice-over, with the purpose of mimicking the literary narrator's depiction of a character or the literary representation of the character's voice and mind. Elliott also points out the common visual representation of texts to communicate a character's literary identity, qualities, alleged origins, and the context that is thus proclaimed to condition the representation of that character (165). Still, unless nothing but a novel's text is projected on the screen, the representations of thematic content unavoidably undergo transformations when stories and characters are adapted from novel to film, especially if there is an ambition for the story and its elements to function well within the new narrative media environment.

For young adults, and others who read for life, a perspective which Martha Nussbaum passionately endorses (230-44), the transformations of thematic content matter, because they affect the grounds for reflection as readers and viewers ponder who they are and who they can become while they engage in fiction. For some, the alterations may cause confusion and resentment, but in this dissertation, I second Suzanne Diamond's claim that the engagement in adaptations, for audiences, "offers a rich metaphorical corollary to the processes ... usually involved in remembering and negotiating "who we are" (102). Because adaptations overtly relate to other narratives, they present multiple and complementary versions of characters in an extended story universe, which is conditioned by the representation of those alternative versions and the transformations of thematic content. Their capacity to serve as aesthetic analogies and metaphors may thereby expand the experience for audiences who are engaged in the everyday processes of personal development and social adaptation. In addition, the screenplays for novel-film adaptations present another version of each narrative, since the screenplay are not just a transitional medium that links other media forms together in the adaptation process, but also carries its own weight as an expressive and representational artwork, with unique qualities regarding characterisation and thematic representation. 
It is in this context that this study asks how the acts of transformation affect thematic representations when the characters of contemporary novels are adapted and migrate to the cinematic world and to our minds. The overarching argument is that engagement in adaptations offers not only alternative versions, but also metaphorical relations between the alternative representations, their expressions, thematic arguments, as well as metaphorical processes of transformation, all of which may be used for audiences as a basis for reflection. Studying the adaptation of themes naturally draws attention to the complexity of adapting ideas and abstract content, which links adaptation and translation studies together. However, whereas literary translators are rarely afforded any substantial creative freedom, screenwriters and filmmakers who adapt a work of fiction commonly do so with distinct creative ambitions, to the effect that the thematic content and meaning is affected. As an appropriate thematic example, the dissertation will focus on the representation of adulthood in novels, screenplays, and films. In this introductory chapter, I will first discuss the didactic perspective, why we engage in fiction, which has influenced the purpose of this project, before presenting the aims of the project, the research approaches and methods, the suggested scope of the dissertation, and an outline of the following chapters.

\section{Why We Engage in Fiction}

The understanding of the social world, as we know it, naturally conditions the construction of fictional worlds, their characters, and events. Likewise, there is no doubt that most fictional narratives are deliberately designed to affect their audiences, and from that perspective they are constructed as "intentional-communicative artefacts," in contrast with the less controlled flow of everyday life (Currie 6). One of the benefits of fictional narratives is indeed that they offer instructional metaphors and vicarious simulative sets of experiences (Oatley and Mar; Nussbaum; Dennet, Vermeule), and they thus have the potential to be both transformative and formative. To a great extent, as Joseph Carroll asserts, fiction consequently presents "simulated 
situations through which we can model our own behaviours" (Literary 159). Similarly, Joseph Campbell's influential The Hero with a Thousand Faces (2008 [1949]) builds the argument that most lasting stories and myths are structured rhetorically to thematise the complexities and themes that circumscribe the idea of being a reasonably whole person in balance with a surrounding community. Since then, James Phelan (1989), Brian Boyd (2007), Christopher Vogler (2007), and Patrick Colm Hogan (2011), among others, have endorsed the idea that we commonly read and watch fiction Hollywood films, novels, complex fiction, and ancient myths alike - with the purpose of realising who we can be or become in the social world. Some engage in fiction with this as a conscious purpose, and other with less awareness of how characters and stories may affect them.

Although fictional characters do not physically enter the social space of the physical world, they penetrate the cognitive space of readers and viewers, and the fictional events thus produce situations and experiences that are stored in the audience's imagining minds (Smith 278; Zunshine 108; Burke 180). This is largely due to the designs of fictional stories, which inherently afford and incite audiences to mentally transgress the borders between the social and fictional worlds for a while. Moreover, most readers and viewers can easily make use of their imaginative capacities to situate themselves in the fictional environments, and see parallels between fictional situations and what they themselves have experienced in the real social world. Fictional worlds, characters, and their story events may thus potentially affect audiences almost as deeply as if the characters had occurred in real social life, scholars like Nussbaum, Dennis Cutchins, and Lisa Zunshine argue. From the Bildungsroman to college films, from chick lit to Oscar winning dramas, fictional stories and their characters may consequently serve as tools for thematic reflections about what it is to be a mature person or an adult.

In this context, Hogan observes that fictional stories tend to represent variations of "universal narrative prototypes" (125) through characters who strive for, preserve, and remain in personal states of normalcy, in which satisfying levels of 
meaning and happiness can be upheld (126). Hogan further submits that these representations form images of a mental "emotional geography" of what it is to be a reasonably stable adult (31). Similarly, Richard Gerrig reasons that the content of fictional stories is generally assessed in relationship to sets of normalities based on a play between the fictional world and the individual reader's, or viewer's, experiences and memories (45). "We form a prototype (roughly, a standard case) by weighted averaging over a set of instances," Hogan explains (47), making the assumption that the goal to attain some happiness in life is best achieved by adaptation to the sphere of life's social normalcy. Hence, while most fictional stories problematise narrow definitions of normal states of affairs, they also demonstrate the need for adaptation.

Admittedly, the use of the terms normality or normalcy can be provocative, especially when it comes to matters of identity, happiness, and life stages, because normality is so often perceived as a narrowly defined and fixed state of being, which leaves little room for any sense of the developed individuality with which the ideas of happiness and adulthood are both associated. Progressive literature and films therefore often question a narrow-minded definition of normality, in line with Anthony Giddens' argument that normality "is more than just a mutually sustained show of interaction which individuals put on for one another" (126). He instead relates normal behaviour to life patterns that enable us to communicate, socialise, and attend to practical matters in reasonably safe and less than completely random patterns. Giddens' definition of normality thus involves degrees of stability, reliability, predictability, and sociability. From a similar standpoint, Franco Moretti suggests a useful definition of normality when he writes about the Bildungsroman as "an unmarked entity" (11), which is not a fixed point but is defined by a flexible range of variations. The way I will use the word normality is never just as a normative and excluding term, because the conditions for normality and the individual's life patterns are always under negotiation. Hence, its definition relies on social context and it is always open to change and to the inclusion of variations of human behaviour which do not pose an overt threat to others. As part of the process of the constant 
redefinition of normality, thematic studies have long focused on the representation of variations that are included in a less judgemental, societal concept of normality. In the following I will also make use of the term normalcy, in line with Hogan's reasoning (30), as referring to the experience of life when situations appear to unfold within the range of what might be anticipated without greater efforts at reflection.

In fiction, characters can find ample room for individuality and autonomy within the spaces of normality, socialisation, and attachments to others in their capacity as fictional beings, and in so doing, they qualify as individual adult persons in the fictional worlds. In other words, the prototypical adult characters ideally embrace an existence within the social order of normalities, while they also affect the constitution of that social order, so that they may find a sense of individual completeness in "social integration as a simple part of a whole," Moretti submits (16). In fact, it seems that a developed individuality actually depends on adaptation to normality as an allowing, organic space, just as the state of adulthood does. To borrow Anita Wohlman's words, films and novels “feed from the culture from which they emerge and simultaneously send out messages about age and ageing to their viewers and readers" $(2014,17)$.

An enabling and inclusive definition of normalcy is also an underlying normative concept in development psychology and sociology, with adulthood as a stage between growth and decline in many social and physical contexts. Clary Krekula shows that age norms function as tools for analyses, negotiations, and the regulation of norms for professionals, institutions, individuals, and scholars (2009). In this context, the notion of an adult human being, competent, able, and reasonably happy, seems to form an abstract norm or point of reference around which ideals and individual visions can be fashioned. While they may of course be read or watched with other emphases and intentions as well, most novels and films, I argue, can thus be regarded as fictions about adulthood based on notions of normalities. This does not mean that the portraits of adults are always ideal in fiction, or that children are always found wanting. In fact, adult characters are often represented as greedy, 
selfish, irresponsible, aggressive, and abusive in many teen stories, and the younger characters often have to adopt the adult, responsible roles in many situations. Thus, just as children in real life can exhibit temporary and singular adult qualities, in fictional narratives non-adults can be used to represent adult social, ethical, and cognitive features, and most adult characters habitually stray from their ideal prototypes of being adults. From this perspective, children, teenagers, and young adults often represent romanticised ideals of what it is to be an adult, but they are less representative of actual adulthood. Fictional narratives about adult characters, however, become relevant as maps of social life, personal judgements, emotional responses, and human actions through the representation of normalities, deviations from perceived norms, and individual possibilities.

In view of such didactic aspects of fiction, Dennis Dutton explains that the imaginative mind imitates fictional characters, their personalities and roles, actions and life patterns, and in doing so makes it possible for us to efficiently try out alternative lives through fiction, in a way which is impossible in real life, since the experience of fictional stories and their characters offers an unparalleled "low-cost, low risk surrogate experience" (110). Similarly, Wayne Booth declares that such "tryings-out in narrative [...] offer both a relative freedom from consequence and, in their sheer multiplicity, a rich supply of antidotes" (485), which also accentuates the potential use of fictional narratives as veiled self-help guides for a better life. Yet some works of fiction, like for instance Gone Girl, are overtly ironic in their approach to adult standards, which accentuates that narratives need to be handled with great care and judgement, if the purpose of reading and watching them is to get indirect guidance on a sound state of adulthood.

In the contemporary social world, however, adulthood is rarely just a matter of reaching a certain age, when a ritual or the passing of a legal threshold confirms a person as an adult. Nor is the transition to being an adult simply accomplished by moving into a home of one's own, getting a job, and finding a partner for life. Rather, it is a social construct, often equated with what it is to be a whole person (Blatterer 
"Changing"), or to be recognised as "full partners in social interaction" in society (Fraser 113). As an effect of the demands, difficulties, and possibilities of everyday life, the state of adulthood has generally been gradually delayed in contemporary Western societies, at least since the fifties (Kloep and Hendry 111). Nevertheless, the concept of adulthood is so institutionalised (Blatterer, Coming 13; Arnett, Emerging 208) that the actual perception of what it means has become vital to young people's identity development as a point of reference (Raymond and Heseltine 199). To complicate matters further, there is no clearly demarcated threshold to adulthood in modern industrial societies, as Vanda Konstam observes, and adulthood is no longer regarded as a fixed state of life. Instead, the road to adulthood has become "an extended and nonlinear process with no clear indication of completion" (Konstam 7). Thus, as sociologist Harry Blatterer puts it, for many of us "the actualization of options is individualized and personal growth, through an individual pursuit, becomes a modus vivendi" ("Changing" 70). This partly explains why the market for so-called Young Adult fiction finds the bulk of its readers among people between 30 and 44 years old (Bowker Market Research, 2012). It is clear that the market for fiction that thematises the conditions of adulthood is expanding, in tandem with the growing general concern with unravelling what it is to be an adult in the contemporary world.

In agreement with Phelan, in Reading People, Reading Plots: Character, Progression, and the Interpretation of Narrative (1989), I believe that it is first and foremost the thematic dimensions, attributes, and functions of characters that make them relevant for their audiences. From this viewpoint, fictional narratives are designed to reflect on and examine various themes, and not just the character and event arcs, which are the dominant aspects in many creative writing manuals. For example, The Writer's Journey (2007), Vogler's influential adaptation of Campbell's The Hero with a Thousand Faces into a creative writing manual, and the step-by-step models of others, based on actions and character developments, diminish the importance of thematic aspects of stories. However, if there is too much emphasis in a film or novel on the characters' 
physical journey through a series of events, and on the protagonist's inner life, thematic issues of universal interest may be reduced to superficial means for narrative conflicts and the individual motivations of the characters. The same can be said about many theoretical manifestos on narratology, because of a focus on the particularities of discourse, as Ian MacKenzie observes. "What narratology has not produced is a convincing account of the essential intuitive leap from a series of events to an underlying theme," he thus asserts (541). As part of this dissertation, I propose that the structure of the hero's journey can bridge that gap to some extent. Conceiving the thematic understanding itself as the hero of a story, the reader or spectator follows the narrative through the plot to construct "meaning in ever larger wholes, to totalize his experience of human existence in time," to borrow Peter Brooks' words (39).

\section{Objectives and Approach}

The primary ambition of this dissertation is to provide a contribution to the study of the thematic representation and transformation in adaptations, as a basis for reflection for readers and viewers, and to consider how this representation is affected when novels are adapted for the screen. Like Elliott (Retbinking 144), I regard the novel, screenplay, and film as equal parts in the composite body of an adaptation, and strive to contribute to the awareness of how adaptations can be of particular use when we, as readers and viewers, struggle with questions about who we are and who we may become. As Anita Wohlmann argues (71), the representation of critical thematic instances regarding the human condition are plotted and orchestrated in accordance with narrative conventions. Some conventions are shared between novels, screenplays, and films, but some are more or less genre or media-specific. Hence, within the scope of this project, I will add to the discussion of the possibilities and limitations that media-specific conventions impose on adaptations and their representations of intricate themes. As a result, I will also present a framework for the analysis of the representation of themes in narrative fiction. 
What happens to the representation of a theme in an adaptation opens up two distinct perspectives: the study of the objects and the study of the processes. To analyse novels and films as objects and compare the different versions rarely produces an answer as to how the transformations were processed or what they might signify. In this dissertation, the study of adaptations as objects is linked to the study of adaptations as processes. Yet, to study the actual adaptation process demands access in time and place to the people who work on a project and to their material. During film production, the numbers of daily changes and of people who are involved in making these alterations are overwhelming. However, the inclusion of the screenplay as an object makes it possible to trace certain changes step-by-step and come closer to understanding the representations in the narratives in terms of the process of adaptation. For reasons I will return to in chapter 2, the screenplay has commonly been neglected by scholars of literature, film, and adaptation. Through this study, I therefore also hope to demonstrate the value of screenplays as research objects, especially in the field of adaptation studies, and as works of fiction in their own right.

Because of its inherent interdisciplinary constitution, adaptation studies is demanding, not least, as Christine Geraghty declares, because most scholars are partial to a specific field ("Intertextuality"). There is thus a risk of imbalance in the research design when adaptations are studied, which might affect the particular observations made, as well as the conclusions that are drawn from them. With adulthood as a thematic example, and with novels, screenplays, films, and adaptation as the main focal points, this project evidently demands interdisciplinary and mixed research methods, as well as specific demarcations.

Firstly, like David Bordwell, I find that fictional narratives are designed and "organized around functional principles" ("Neo-Structuralist" 205), in particular to communicate and to be part of a systemic cognitive production of meaning. I acknowledge that a film adaptation can be seen as a product of the novel it is based upon. Yet I also understand that adaptations are "autonomous works in their own 
right," as Geraghty posits (Now a Major 7), and that a novel-film adaptation can be regarded as a composite of both objects and the relationship between them. From a functional viewpoint, with the use of adaptations in mind, none of these views should be excluded. Although they might be part of an evolutionary process by which one evolves from another, the novel, the screenplay, and the film co-exist, rather like clones with a mutual yet individual DNA string, as Gary R. Bortolotti and Linda Hutcheon argue. From this perspective, I find Sarah Cardwell's notion of the ur-text useful, as a mythic story that all versions of a narrative represent from different perspectives, because it allows the individual works of fiction to be studied individually and in relation to each other at the same time. The ur-text notion, with its web of versions, also invites the imagining of a greater whole, or design, as a result of the cognitive flicker of meanings from alternative narratives, which is of value when the construction of thematic meaning is in focus.

Secondly, narratives are designed to function and to serve a purpose within a context. As Bordwell declares, "neo-structuralist narratologists of film largely avoid discussing function" ("Neo-structuralist" 204). I recognise that the narrative models that Campbell, with his Jungian take on myths, the neo-formalist Kristin Thompson, and the cognitive, evolutionary narratologist Hogan present originate from different theoretical foundations. Yet they all share a functional, neo-Aristotelian connection, and add relevance and practical frameworks to Phelan's rhetorical and thematic approach as he regards characters as designs with thematic purposes.

Third, besides narrative and sociological structures, I also make use of a set of markers of adulthood in this dissertation. They are constructed as an amalgamation of various sociological and psychological models, and serve as a framework for the semantic categorisation of various character attributes that can be related to adulthood in novels, screenplays, and films. The objective is not to present a new definition of adulthood that can compete with existing theories in those fields; I only suggest a tentative framework for the study of adulthood in fiction, which serves the purposes of this study. I thus employ semantic categorisation of characters' mental 
and physical actions and reactions, which can be productive for thematic studies, especially if they are used to indicate qualitative patterns, aspects, relationships, and processes as a foundation for analysis. Yet, to use structural principles for semantic analysis demands a reflexive ideological awareness in the analysis, since ethical values and functional relationships determine the judgements and "qualifications" of characters' actions (Bal 131). There is also a risk that structures may reduce the complexities of the qualities of the objects and phenomena that are studied.

Fourthly, since most scholars traditionally associate adulthood, as a theme, with either mature-age studies or the representation of the younger, formative years of a person's life, I should clarify that the use of adulthood as an example theme in this dissertation concerns the representation of what it is to be adult, not of what becoming an adult is about, particularly in connection with the thematic transformations and simultaneous representations of themes that media adaptations result in.

Fifthly, I only include what could be regarded as successful novels, screenplays, and films in the study. Likewise, I have limited my scope to exclude adaptations of novels written before the year 2000. This year is, of course, chosen with a certain arbitrariness. Its symbolic value as a representation of contemporary culture is, however, significant, as events like 9/11, the Me Too movement, and other phenomena since 2000 have radically affected the cultural climate in which novels, screenplays, and films are produced. The selection is also based on fiction written and produced in the English language. That being said, American and British perspectives dominate, and while Kira-Anne Pelican et al., Hogan, Campbell, and Boyd indicate that the thematic organisation of character traits and narrative structures are universal in successful stories, I cannot assert that any conclusions regarding the representation of adulthood are decisively valid outside the delineations of this project. Moreover, I have focused on the protagonists in the stories, not on the secondary characters who to some extent define them, although that could have been an interesting and complementary approach. Given these limitations, I will be 
restricted from drawing any too far-reaching conclusions regarding adaptations in general. Conversely, because the project mainly revolves around certain fiction titles, with specific novelists, screenwriters, and directors, the thesis will naturally add to our understanding of the characterisation, the representation of adulthood, and the rhetoric in those works.

As stated, this dissertation will primarily provide insights on how the representation of characters and themes is affected by the novel-film adaptation process, on some of the transformative processes that are involved, and thus on the didactic opportunities that adaptations may offer, when audiences engage in the thematic representations and processes of adaptation of contemporary novels, screenplays, and films. Furthermore, the thesis will suggest a way for fidelity studies and comparisons between films and novels to be productive without the influence of judgements, thereby advancing our comprehension of the adaptation of the spirit of stories, to speak with Colin MacCabe (8). In particular, these insights have implications for the pedagogy of film, screenwriting, and literature, since they have to do with the uses of fiction.

Since this project asserts that the screenplay is a centrepiece in the novel-tofilm story world and its transformation, the project aspires to contribute to the foregrounding of the screenplay in academic research and in adaptation studies in particular. For screenwriters, the findings may be of practical use when characters and themes are to be adapted in an environment of converging media and story landscapes. The models I present can hopefully be used as functional tools to communicate ideas when novelists, directors, screenwriters, and producers bring their respective discourses and motivations to the table. Moreover, the project also connects to the growing field of age studies, and might be of interest for practitioners and scholars in areas linked to psychology and sociology, although the findings of this thesis might be of greater potential value for teachers who use film and literature as educational elements in their classes. 


\section{Chapter Outline}

In the following, chapter 2 outlines my position in relation to adaptation studies, beginning with a brief discussion about media specificity, before the poetics of the screenplay is addressed as an essential background. An overview of central approaches to adaptation studies is then sketched in relation to the transformation of characters and themes, before I focus on non-linear models of adaptations and the possibilities involved in studying characters as fictional beings and migrants. To conclude the chapter, I address the bi-directional process of adaptation and appropriation that conditions the reception and creation of novel-film adaptations. Chapter 3 addresses thematic representation in fiction in general, with a discussion of theories and models of studying themes as they are represented by characters in fiction; this leads to a consideration of the plot as a rhetorical line of argument in narrative fiction, based on the structure of the hero's journey, or the monomyth, as it is presented by Joseph Campbell. I then turn to the concept of adulthood and its representation in fiction, since it constitutes the thematic example in this dissertation, and outline a model of markers of adulthood that can be used to study the representation of adulthood in fiction, based on studies of adulthood, in the fields of sociology and psychology. Since this is an article-based dissertation, it includes and is based on five articles. The full-length articles are attached in appendices A-E. Chapter 4 provides a summary of the five articles and a discussion of their individual contributions, as well as how they, as a unity, address the overall research aim. In the concluding Chapter 5, I consider the implications of this dissertation as a whole, with regard to the understanding of what happens to the representation of adulthood, and themes in general, when novels are adapted for the screen, and how those transformations and their results may be used as means for thematic reflection. The theoretical and methodological consequences in relation to the study of adaptations, literature, screenwriting, and film criticism are also summarised. Finally, the practical implications of the models that this project has resulted in will be discussed, mostly in relation to teaching and creative writing. 


\section{Chapter 2}

\section{Adaptation, Appropriation, Migration, and the Screenplay}

Before I address how thematic content is represented through characters in fiction, I will outline my position in relation to adaptation studies in general. Initially, the question of media specificity will be discussed briefly in the context of thematic representation. Numerous publications have covered how and why novels and films are not the same, from a variety of theoretical perspectives; however, these cannot all be covered here, however interesting they may be. For in depth discussions of media-specific fallacies, I am indebted to Thomas Leitch's article "Twelve Fallacies," to Elliott's Rethinking the Novel/Film Debate, and to her overview in "Theorizing Adaptations/Adapting Theories." It is also of great importance to explain the position of the screenplay in relation to the adaptation process and to adaptation scholarship, since this has been widely neglected by scholars in the field. In so doing, I will also introduce the poetics of the screenplay, which is perhaps the narrative form that is most circumscribed by formal conventions. Finally, I discuss some linear and non-linear approaches to the study of adaptations, before I focus on the interplay between adaptation and appropriation, and the benefits of regarding characters as migrants when thematic issues are studied in connection with adaptations.

\section{Media Specificity}

Many debates on adaptations end up making judgements or explanations based on media-specific qualities. Seymour Chatman's article "What Novels Can Do That Films Can't (and Vice Versa)" from 1980 is an example of this, and an illustration of what Leitch defines as the first phase of the history of adaptation scholarship ("Introduction" 2). The study of adaptations has been dominated by the focus on works of fiction which have gained the status of literary masterpieces, and on their film adaptations. This focus has produced a biased notion of the "ideal constructs" (Bazin 49; MacCabe 17) of their cinematic representations in the minds of the critic or scholar, which has conditioned much of that generation of adaptation criticism, 
in stark contrast to the fact that few novels or films can be said to have been produced under ideal conditions. Disregarding the actual range of experiences that demonstrate what can be done with different media, critics still use examples of how novelists and filmmakers have failed to achieve something, for the sake of arguing that certain things cannot be done. However, a poor film adaptation does not mean that a certain novel is unfilmable, just as the less successful novels that have been adapted to successful films - as is often the case with Alfred Hitchcock's and Stanley Kubrick's films for instance - do not prove that the stories, their characters, and themes are unsuitable for the novel form. To put it plainly, it is not the novel that produces the film.

In general, from a functional perspective and with the reader's and viewer's understanding in mind, I side with Dudley Andrew, who states that, theoretically, the same narrative elements and codes, such as characters, events, contexts, themes, and so forth, can be represented and their meanings communicated, regardless of narrative media, if they are produced under ideal conditions (103). Elliott has convincingly shown how the communicated content of a narrative is not bound by form, due to the analogue possibilities of different narrative media (Retbinking). As Hutcheon argues, the content may hypothetically be transferred to a new form (10), but only if the execution is performed under completely ideal circumstances. What thus constitute the most definitive distinctions between different versions of stories are the means of expression that are applied and how these are coordinated to produce a foundation for the audience's meaning-making process.

With regard to the notion of an ideal construct, it can be observed that much of the writing on media specificity takes as a starting point the idea of the purity or essence of each narrative medium, ignoring the fact that there is no such thing as a purely novelistic or cinematic means of expression, only typical mixtures of various modes, as Cardwell and Anne Gjelsvik, among others, suggest. The first wave of adaptation criticism nevertheless kept an insistent focus on the division between the verbal and the visual, as well as between the linear representation of words and 
the cinematic spatial representation. For instance, in Novel to Film (1986), Brian McFarlane defines novels as linear, on the grounds that the words are usually read in a distinct order, which, he asserts, makes the progression of the meaning-making process linear "through a gradual accretion of information," whereas films are defined as spatial because they mostly present parallel tracks of visual, verbal, and aural signs (26-27). With reference to Roland Barthes, McFarlane also argues that novels work entirely symbolically, through the telling mode, since each word has to be conceptually decoded, whereas films are understood perceptually, because meaning is presented rather than told.

Even more problematically, McFarlane claims that films cannot express action in the past. In contrast, it is reasonable to contend that films cannot present anything but representations of the past, since films show staged sequences of a story which have already occurred and been recorded long before a film is shown on the screen. In addition, the events represented have already occurred, in the screenplay before they are filmed, where they are preserved in a frozen present tense. However, both these positions disregard how films are perceived by audiences. Over the years, film conventions have developed means and linguistic conventions to signal past tense to their audiences as clearly as any verbal grammatical tense might do. Furthermore, from a perceptual and phenomenological vantage point, the multi-literate readers and spectator can be transported, in their imaginations, to the now of the past when a narrative so demands, and are able to understand the present and the past in context, regardless of whether they are reading a novel or watching a film.

Another aspect of the ideal constructs of a fiction film or a novel is that they involve a controlled experience at the cinema or an ideal reading situation, which seems to take place at home, with pauses, without external interferences, and with a reader who on occasion turns back the pages to return to already read sentences, paragraphs, or sections. However, there is no way to ensure that a reader does not skip a specific word or paragraph. Neither can the reader's attention be guaranteed. In fact, on average a third of the words in a novel are probably skipped by readers 
(Rayner 2009), as if there is a limit to the number of words the average reader can take in or desire for an understanding of the text.

In the context of perceptual overload, it is nevertheless the audiovisual multitrack constitution of the film medium that is most commonly foregrounded, with spoken words, music, sound, set designs, movements, and so much more on display in every frame. Chatman thus notes that because films commonly present countless pieces of information at once, and spectators rarely have the time to consider them all, the "pressure from the narrative component is too great. Events move too fast" ("What novels" 126). Similarly, McFarlane addresses "the simultaneous bombardment by several claims on our attention" in films (28-9). However, Robert Stam, in the introduction to Literature to Film (2005), turns the argument around and argues that, although films may be open to obvious contradictions as an effect of the multitrack phenomenon, they have the capacity to produce "multitemporal, polyrhythmic" complexities (60). Through the habits that streaming services, digital discs, and home cinema systems have produced, it has been made apparent that the above-mentioned limitations and possibilities rather depend on artistic production and distribution forms than on media-specific qualities as such.

Hence, the past two decades of adaptation scholarship have resulted in a more nuanced view of media specificity. Elliott has demonstrated how the different signifying systems are present in both novels and films, which further complicates the form/content dilemma for the theoretically minded (Retbinking). In prose, novelists paint pictures for the mind and the reading process inscribes them as visual memories, just as clearly as the words and linguistic representations are created in the mind to store the audiovisual expressions in a film. The oft-repeated distinction between the telling and showing modes of film and literature have thus been dismissed as possible yearnings for pure media forms (Leitch, "Twelve Fallacies" 151), but, in spite of the ever present signs of the contemporary convergence culture, in which we may drink Austen experiences from a Mr. Darcy cup, or play a Game of 
Thrones board game, the inclination to reduce the potential of cinematic and literary expressions and forms to media essences prevails in many of our romantic minds.

Historically, the distinction between showing and telling can be traced to the intertitles of early cinema, which were often used as simple solutions to a narrative problem; media students are still told not to use voice overs in their films, because they compromise the film medium. Thus, Chatman's statement that "it is not cinematic description but merely description by literary assertion transferred to film" ("What Novels" 440) still carries weight. Nevertheless, the film ranked highest by audiences on IMDB has for years been Shawshank Redemption (Darabont, 1994), a film which relies heavily on the narrating voiceover. So, again, it is not a matter of whether a film is cinematic or not. What is relevant is the purpose that the expressive means fills, and that is realised in a film production.

Much could also be said about the narrating levels and perspectives in novels, screenplays, and films. Anne Igelström's dissertation Narrration in the Screenplay Text (2013) and Murray Smith's Engaging Characters (1995) give sufficient evidence to say that the same narrational strategies apply regardless of medium. Whereas novels may use first, second, and third person narration, films use the camera and sounds to place the eyes and ears of the spectator in similar positions to those the novel does in relation to the narrator, regarding narration, focalisation, and reader identification. In the end, as Rick Altman theorises in A Theory of Narrative (2008), readers and spectator above all follow characters' minds, actions, and perspectives through a narrative. Whom someone follows may change during the progression of a narrative, from protagonist, to antagonist, to the exchange between them, and to the narrator, although most narratives are primarily structured to facilitate the following of a protagonist through a narrator, who carries the heaviest thematic burden, especially since readers' and spectators' minds cannot be entirely controlled.

When it comes to characters, it is often argued that films depend more on clear behavioural stereotypes and coherent, less complex characters, unlike novels, although these appear in popular novels to the same extent that they do in popular 
films. Thus, like most adaptation theorists, Hutcheon regards it as a faulty cliché to say that the representation of the complex emotions and thoughts of characters is exclusive to text (56; see also Leitch "Twelve Fallacies" 158). It is true that, during the progression of the experiences of the narrative, the gradual development and the spontaneous analysis of a character often allow more time for the reader than for the film viewer. As previously indicated, this is often compensated for by the use in films of the rich parallel modes of impressions in terms of sound, words, and visual information. The narrator's voice and the reporting of thoughts and emotions in literature also occur in films. Contemporary cinematic characters often tell audiences directly or indirectly about their thoughts and emotional states, so the inner life of characters can be powerfully represented and understood by spectators through a vast arsenal of expressive means, from camera work and editing, to speech, voiceovers, actions, and not least through pure acting and characters' interactions and interrelations, the latter based on the same decoding strategies as those that we use in the social world to sense and understand what someone feels or thinks, which is known as the theory of mind. André Bazin's comment on The Pastoral Symphony is illuminating on this point:

But I do think that Michèle Morgan's beautiful eyes [...] are able to communicate the blind Gertrude's innermost thoughts ... All it takes is for the filmmakers to have enough visual imagination to create the cinematic equivalent of the style of the original, and for the critic to have the eyes to see it. (20)

In consequence, there is little support for the opinion that the possibilities of employing characters' thematic dimensions and functions are restricted by the film medium. The greatest difference between novels and films concerning the representation of themes through characters is perhaps that live action feature films still depend on actors and actresses. These performers bring the illusion of corporeality to characters on the screen, and sometimes they come with thematic baggage from previous appearances and from their physical appearances, as Stam 
observes (10-11), which is often considered, emphasised or compensated for in the casting, acting, direction, camera work, makeup, the digital editing of bodies and faces, and even lighting.

To conclude this brief but necessary discussion on media specificity in relation to novels and films, themes may perhaps not be "the easiest story elements to see as adaptable," as Hutcheon claims that they are (10), but they are definitely not mediaspecific. However, because films are usually far more expensive to produce than the average novel, they are riskier ventures. Producers may very well impose thematic restraints, with or without a production code, but this is also known to happen when editors comment on manuscripts for novels too. Gjelsvik reasons that some scenes are visually sensitive, whereas others are linguistically provocative, and that "we tend to react differently towards different art forms due to a combination of medium characteristics and conventions" as an effect of their emotional impact (247). That said, the question remains as to whether conventions in literature, novels, and films and their "traditions of quality" (Francois Truffaut in Leitch, "Twelve fallacies"162) affect the rhetorical presentation of adulthood differently.

\section{The Status and Poetics of the Screenplay}

With regard to media specificity, the screenplay is a hybrid, part literature, part film, but still circumscribed by its own formal conventions, distinct from those of the novel, the film, and the theatrical play for that matter. Strangely enough, in spite of the recent expansion of topics addressed in adaptation studies and the fact that screenwriters and screenplays are imperative for film adaptations, the screenplay has not yet been fully recognised as a natural and integral part of adaptation, literature, or film studies. As Steven Price observes, texts on adaptation still rarely mention screenplays, unless just to credit the director or screenwriter, or to comment fleetingly on the production process (Screenplay 57-58). When screenplays are occasionally analysed, they are more often than not equated with the structures of events, settings, and dialogues in the relevant films (110). Voters for the Best 
Screenplay category for the Academy Awards, the Golden Globe Awards, and even the Writers' Guild Awards rarely read the actual screenplays, but evaluate them by watching the films. Thereby, they too fail to acknowledge the screenplays as works with values and qualities greater than those once afforded to them as subordinate blueprints for the films.

Still, screenplays have recently been given some serious scholarly attention and have been addressed as valuable resources in adaptation studies (Cattrysse 25). Leitch, for one, endorses the view that both novels and screenplays can be regarded as source texts for film adaptations, and that adapted screenplays are as much a result of an adaptation process as adapted films are ("Twelve Fallacies"). With the classic source-target model in mind, the adapted screenplay can thus be regarded as being both source and target. Beyond the prescriptive and less academic screenwriting manuals, Claudia Sternberg (1997), Peter van Stapele (2005), Kevin Alexander Boon (2008), Igelström (2013), and Price (2013) are a few of the scholars who have laid the ground for a narratology of the modern screenplay. Since 2010, Journal of Screenwriting has also contributed with insights on the philosophy and history of the screenplay, the process of writing, and research on screenwriting manuals, which may in time enhance the literary status of the screenplay.

However, screenplays have long been denied the status of literary objects as an unfortunate consequence of their standardised format and function as "nothing more than a set of notes to a production crew" (Luttrell 10). For instance, Noël Carroll (2008) claims that a screenplay cannot be regarded as an independent artwork, since it is above all a production tool and cannot be attributed to a singular creative mind (Philosophy 69). Similarly, Ian W. Macdonald (2013) argues that a screenplay is an integral part of a film project, and as an object represents a collaborative "screen idea" for a film, which in that context is the one and only artwork at hand (89). In spite of many screenwriters' status as literary stars today, this view lingers, and screenplays are seen as descriptive structure drawings or blueprints. It is thus implied that the screenwriter should entrust the artistic initiative, that is to say, all decisions 
about aesthetics and depth of meaning, to the directors, photographers, actors, and others.

Still, in contrast to the blueprint metaphor and the notion of the screen idea, Ted Nannicelli contends that screenplays should have the same status as art works as plays written for the stage, since staged performances and films are in fact implementations of their scripts (409-10). Nannicelli recalls Gus van Sant's 1998 remake of Psycho, which was produced with the same script as used by Hitchcock's 1960 film (409-10), to exemplify that a screenplay, like a play written for the stage, is a literary artwork, and that films, like staged plays, are performances of screenplays. There are numerous other examples in film history of this type of productivity. Just to mention two examples, Leo McCarey made two different films from the same screenplay (Love Affair, 1939; An Affair to Remember, 1957), while Michael Haneke translated his own Funny Games (1997) from a German to an English version with British and American actors in 2007. This indicates that screenplays should indeed be regarded as texts that call for artistic evaluation and interpretation.

The screenplay's status as a work of fiction can also be validated through its increasing audiences. As he interrogates the status of the screenplay as text and work of art, Nannicelli thus observes that

when we browse in bookstores these days, it is not uncommon to find more published screenplays in the film section than published theatrical scripts in the drama section. There are numerous publishers and imprints that now publish classic and contemporary screenplays ... In addition, there are fan communities in which members share both screenplays based on previously existing television shows, movies, and characters, as well as original screenplays. These fan screenplays are not intended to function as production documents, but rather are created to be read for their own sake by community members. (408)

If the various roles and functions of a screenplay are embraced, as I have sketched above, the adapted screenplay appears to be more than a singular literary text. This 
is the case in a more concrete way as well. In the course of a film project new versions may appear by the hour (Price, Screenplay 69), so it is often difficult to locate one singular product that can be named The Screenplay, and exclude all other versions of it. Instead, the screenplay seems to be in a state of constant flux and transformation, as Sternberg observes, until it has lost the original function and meaning that it had when it was first presented to its intended readers. It thus materialises the palimpsestuous nature of all fiction.

However, to simplify, the first version may be considered the writer's original screenplay, a version which is presented to producers before the rest of the film production is fully launched. The second is a text that has been reworked to function as an initial raw material for the director and the production team (Boozer 6). In the process, various production conditions, ranging from legal issues to geographical requirements, demand revisions before a version can be presented that roughly corresponds to the film. Later, a "legal version" (Price, Screenplay 72) or "release script" is produced, which is a strict record of the film for professional use. The most tangible version of a screenplay is a distinct published literary item intended for reading, with commentary texts and images from the film and production set. However, these publications take different forms and may also present the readers with the writer's version, as a shooting script, an edited version to the fullest extent possible consistent with the distributed film, or any other version of the screenplay (Price, History 231). Regardless of which of these is the case, the published screenplay is a literary work in its own right, and, when it is an adaptation, it functions as a possible interpretation of and commentary on both the novel and the film.

As indicated, as a result of industrial demands, a screenplay has its set format and unconditional poetics. As manuals have stipulated, at least since Field's Screenplay in 1979, screenplays are regulated by strict conventions. ${ }^{1}$ To begin with, a screenplay should still be written using the Courier font, although the digital era has introduced

\footnotetext{
1 See Price's $A$ History of the Screenplay for an account of how screenplay conventions have gradually emerged.
} 
alternatives, and one page of text is conventionally written to correspond to one minute of reading and film. To compare, a two-hour feature film comprises around 25,000-30,000 words (Field 22), while a novel often contains over 100,000 words. McCarthy's short novel The Road consists of 58,722 words, whereas Penhall's adapted screenplay contains 25,179 words. Meanwhile, McEwan's novel Atonement conforms to the novel format with its 123,842 words, and Hampton's shooting script contains 18,740 words. This means that adapted screenplays condense the novels that precede them. Therefore, Boon argues, the screenplay text should be associated with poetry, since it is characterised by the same "concise and connotative" qualities (17). In the case of adaptation screenplays, they are often constructed to bridge the poetics of novels and films.

Furthermore, the screenplay is strictly divided into scenes, "defined by continuous action in one place" (Price, History 210). Each scene has a pragmatic heading specifying interior/exterior setting, specific location, and time of day. It might be followed by a brief depiction of action and setting, which demands a linguistic and poetic condensation and exactitude to meet the requirements of the format. To accomplish this task, the screenwriter makes use of three modes: a descriptive mode, outlining the mise-en-scene or the necessary details of the physical setting; a report mode, relating the sequence of actions or movements; and a comment mode, giving the writer room for additional information that is absolutely necessary to understand the scene (Sternberg). Apart from this, conventions advise that any speech directions, and other specifications, should be omitted. Characteristics of the characters and places must not be too specific, since they might not be matched by real life appearances. Price remarks that this helps readers to set a so-called master screenplay apart from a shooting script, which often has contributions from the creative members of the actual film production team. Nevertheless, screenwriters habitually break some of these rules in every screenplay.

Furthermore, as Steven Maras notes, the screenplay emphasises the reader's specific placement in the text, which is distinct from that in both plays and novels. 
Frequently, what happens in a screenplay is described with the words we see this or that happening, with the manifest purpose of guiding the readers' and potential spectators' experiences (67), and inviting the reader to fill gaps and interpret. This inclusion of the reader as a voyeur in the text thus positions him/her between the text's implied reader and the characters, which creates the illusion of the reader as a spectator who experiences the fictional world as if being there. Therefore, Igelström maintains, a screenplay is obviously also a piece of literary fiction, which gives rise to three separate narrating voices: the extra-fictional voice, responsible for production values, and the impersonal fictional voice, which is merely responsible for narrating the fictional accounts, as well as the personal fictional voices of the characters.

In contrast to Kubrick's statement that the screenplay is the most noncommunicative form of writing ever designed, and entirely based on the filmmaker's needs (Maras 63), it should, finally, be emphasised that a screenplay communicates with the ambition of evoking multi-modal mental experiences of text, body, sound, light, smell, space, and so forth, which makes it a text that requires sensory imagination. In reading an adapted screenplay, an interaction is possible with the fictional world of the novel and that of the film, as if there are three parallel worlds that are part of one single story universe. Add to that the readers' personal inferential ambitions to create meanings and purposes attached to their own world, and a very rich communicative game enfolds. In this game, as Maras proposes, the reader of a screenplay becomes a translator, adaptor, and contributor, who rewrites the text to images, and the images to text (70). From this perspective, the screenplay is indeed at the heart of the adaptation process.

\section{Adaptations: The State of Affairs}

Adaptation studies resides in a liminal space. Torn between the openness of intertextuality and cultural and social aspects of texts, cognitive, response-related narratology, and the formal or semiotic approaches to media-specific aesthetics, the research community persistently struggles to keep normative fidelity studies and 
value judgements at bay. The romantic idea of the sacred original, enhanced by readers' emotional memories of the imaginations of worlds that novels produce, has long afflicted adaptations with pejorative judgements. In the comparative tradition, quality-labelled novels have habitually been evaluated against and regarded superior to film versions with regard to structure, content, and the complexity of political or thematic content. Thus, verdicts have repeatedly been passed on the possibilities of communicating a specific story and its content in new media forms, based on its success with audiences or reviewers.

One of the crucial problems, regardless of the approach to adaptations, is that they include processes of change, as well as, commonly, at least two distinct cultural products, such as a film and a novel. Thus, as Elliott argues, "adaptations teach us that theories cannot predict or account for adaptations in all times and places ... because adaptations are always changing and adapting. Any theory of adaptation must ... therefore itself incorporate process and change" ("Theorizing" 34). However, it is vital to understand that an adaptation means development but not necessarily a complete transformation. The Oxford English Dictionary defines an adaptation as "a reproduction modified to suit new uses," and adaptation is ideologically linked to evolution or development, phenomena that enable organisms to survive in new cultural environments. Media adaptations, too, are generally equated with "the term Darwin uses to stress that the structure of an organism is a function of its environment," Colin MacCabe notes (3). In essence, adaptation thus refers to the process of changing a structure, form, or function to fit, or suit, different conditions and environments (Field 323).

Traditionally, most models of adaptation are linear, based on the perception of time and progression. Thus, adaptations are intuitively regarded as one-way transportations, from a source text to its remediation in a different media form. This dated model is based on the material process of adaptation, and entails the problematic idea that there is an entity, in shape of a static, unified source text of permanent meaning, which exists before the adaptation process takes place and from 
which selected core features can be preserved when the new shape of the narrative is formed (Cardwell 13). As a result, there is a persistent illusion, as Pascal Nicklas and Oliver Lindner suggest, of a "mysterious transubstantiation," so that the contents of a novel can be "transported to the new form in a way that leaves it still recognizably the same" (2), which is rarely the case.

Still, there is indisputably always an element of faithfulness, or a cooperative benevolent relationship, both to what has been and to what is to come, in connection with adaptations. Emphasising the relationship to the first version of those that are compared, Rachel Carroll even argues that "all adaptations express or address a desire to return to an 'original' textual encounter," and that adaptations signify a desire to repeat, but also that "every 'return' is inevitably transformative of its object" (1). As Geraghty puts it, adaptations thus entail "an accretion of deposits over time, a recognition of ghostly presences, and a shadowing or doubling of what is on the surface by what is glimpsed behind" (Now a Major 195). Likewise, in Screen Adaptations: Impure Cinema (2010), Deborah Cartmell and Imelda Whelehan propose that "there is still a trace of yearning, of a desire for simplicity and disciplinary purity" (13). I venture to say that this dilemma, which relates to the state of adaptation studies, is equally relevant to cognitive, literary, and film studies and perhaps even more so to the human condition: we are aware of the complex constitution of our realities, but dream of a simple model, based on our preferences, to take us through it all. After all, adaptations allow us not only to refer back, but also to observe alterations, environments, and relationships, and to look forward.

Yet, the conflict between the desire for simple, pragmatic models and the complexities of real life, or artistic and communicative relationships, has taken adaptation studies to the point where competitive concepts, like remediation, intertextuality, intermediality, and multimodality, to mention but a few, are used to position scholars and models in opposition to each other (Elliott, "Theorizing" 23), when they actually share a common ground. As a result of these more open-ended approaches to adaptations, the question of what is and what is not an adaptation has 
been brought to the fore. Together with cognitive and narratological approaches to adaptations, scholars have demonstrated the interconnectedness of works that do not in any way signal overtly that they are adaptations, which has led Hutcheon to ask the pertinent question "what is not an adaptation?" (170). In many ways these are creative approaches to theory, and, as Leitch and Elliott probably would, I say so in a positive sense, because new perspectives challenge our presumptions (see Leitch, Against Conclusions), although I personally direct my curiosity mostly to film adaptations and the novels that share their titles.

One of the more important outcomes of the numerous case studies and theorisations in the field is the rich variety of taxonomies or classifications of creative acts and relationships between adaptation versions. Andrew suggests "borrowing, intersecting, transforming" (98), while Geoffrey Wagner proposes "transposition," "commentary," and "analogy" (222), and Julie Sanders makes an extensive list of other terms that different scholars have used, of which "version, variation, interpretation, continuation, transformation, imitation, pastiche, parody, forgery, travesty, [...] transposition, revaluation, revision, rewriting, echo" are but a few (18). These classifications are clearly reductive if isolated, but most taxonomisers maintain that there is no such thing as purity or an absolute and singular original in an adaptation process. Their purpose is not necessarily to classify adaptations, but to illuminate phenomena in the processes, to expand insights into the complexities of novel-to-film adaptations and appropriations, and to offer new tools and perspectives.

Consequently, returning to Hutcheon's assertion that anything might be considered an adaptation, Ian Q. Hunter picks up the debate and asks "what is only an adaptation?," arguing that even the most explicit one-to-one adaptations abound with connections to other narratives, which are implemented through various modes of adaptation (66). There is no conclusive response to these thought-provoking questions and there is still no comprehensive theory of adaptation taxonomies in place. Nor is it within the scope of this project to approach that enterprise. I settle 
for the observation that in spite of all the calls for new models and theories that so many taxonomies are partly meant to serve, it can be noted that most of the terms rely on the linear relationship between two versions, which indicates the dominance that such a notion still holds quantitatively in adaptation studies, no matter how many other lines of reasoning have been convincingly theorised and advocated.

Indeed, Cartmell and Whelehan argues that every object and process of adaptation comes with "an entirely new set of relations" (Screen Adaptation 22); these come with an infinite number of variables involved in each case, as MacCabe adds (8). For new ways to approach such problems, and thus to challenge the habitual patterns of adaptation scholarship, Leitch, Cutchins, Lawrence Raw, Simone Murray, Cardmell, Whelehan, Elliott, and many others have turned to pedagogy, sociology, historicism, science of business, and other academic fields that focus on multivariable and less-than-linear processes, some of which have already been mentioned. The contribution of this cross-disciplinary curiosity is that it has opened up the field for new models and studies. Nonetheless, regardless of strategy, ambition, motive, or approach, contemporary scholars still find it necessary to continually re-establish the position of adaptation studies in relation to the traditional literature-film and form-content divides, predominantly to support equality between the media, with the meaning-making processes of readers and spectators in mind.

\section{The Attraction of Adaptations}

At this point, I would like to return to the matter of the attraction that keeps the general interest in adaptations so vibrant. In chapter 1 I referred to the analogy between comparing versions of fictional character or story identities and figuring out who we are and can be. Suzanne Diamond maintains that self-negotiating involves how we believe others see us and submits that non-judgemental fidelity analyses "constitute important cultural identity work," because we negotiate a collective version of our self-identity in reading and watching adaptations (105). The mere activity of adaptation engagement thereby enables us to consider our own adaptation 
processes. We may accept that invitation or pay no attention to it. If we do activate the engagement, we may never want a story to end, since, as McFarlane suggests, both novels and films introduce us to incomplete realities that make us want to know more ("Reading" 20), and adaptations add the joy of possibilities that the variations signify (Hutcheon 9), concerning not just the characters in the stories but also ourselves and the world. Adaptations thus represent the incompleteness of our consciousness, and suggest that, with continual efforts, we may one day see our curiosity about ourselves satisfied. Perhaps, then, if the end of a story is regarded as a metaphor for death, adaptations represent our dreams of eternal life.

In a similar line of reasoning, MacCabe stresses that "the source text is part of the appeal and the attraction of the film" (5), but this, I would argue, might not be due to the dream of an eternal identity that a source text might represent, but rather to the demonstration of the possibility of adapting to new conditions. As such, an adaptation distances the experience from the idea of a "divine design" and individual essence, MacCabe argues, and replaces it with "a continuous process" (3). Sanders remarks, in agreement, that "the Mendel-Darwin synthesis offers a useful way of thinking about the happy combination of influence and creativity, of tradition and the individual talent, and of parental influence and offspring in appropriative literature, perhaps all literature" (156). By the same token, from Moretti's and Erikson's point of view, the dialogical and intertextual approaches to identity correspond to the human drive to find a balance between individuality and communality, between difference and sameness, which is the upside of Frederic Jameson's proposition that the appeal of adaptations lies mainly in the inherent "allegories of their never-ending and unresolvable struggles for primacy" (232). In the end, and in spite of the subversive tendency of adaptations to undermine power in their claim for renewal, which Hutcheon identifies, adaptations fulfil the human need or desire for patterns and development (175). We may therefore be intrigued by the relevance of a character, story, or theme that crosses borders of culture and time, in search of something that links the externals of their lives and our own to the 
internal, "bringing together the comfort of ritual and recognition with the delight of surprise and novelty" (173).

\section{Non-linearity}

The line of criticism that breaks most thoroughly away from linear and hierarchical models is involved with audiences' ways of relating to and understanding fiction. Henry Jenkins' ground-breaking observations on audience activities and processes of making sense of and living with fiction imparted a severe blow to those who maintain narrative hierarchies and argue that reader response and audience research should be confined to sociology and psychology departments, or claim that such methodologies cannot reveal anything substantial about the texts. For readers and spectators, the hegemony of the author and the signification which is restricted to the boundaries of textual and audio-visual signs have been productively abandoned. Derrida's postulation "il n'y a pas de hors-text" - there is nothing, or no, "outside the text" comes to mind (158). He asserted that when a narrative was based on other accounts of events there was nothing outside of these textual representations to relate to. Conversely, if all we know about reality, as Jean Baudrillard and Edmund Husserl suggest, is inferred from representations of reality, there is no outside the text, only mystories, to use Greg Ulmer's phrase (2004), and the scholarly supremacy of deciding what a text means must at least allow for alternative truths, if only in this matter. As I will argue in what follows, for adaptation characters there might not be any outsidethe-text at all, since these characters have a tendency to link texts together in the most creative ways, and they may very well reside in an all-inclusive universe.

This attention to the use of fiction and how meanings are experienced and construed brings to mind that people may watch the film, or TV show, before they read the book, as Hutcheon points out (xvii). In such instances, the film precedes and informs the person's understanding of the novel, no matter when the publication and release dates are. In some cases, a novel may be published after the film premiere, 
like Arthur C. Clarke's novel 2001: A Space Odyssey, ${ }^{2}$ while in the case of Game of Thrones the final season could be said to be based on a novel which was not yet written. To upset the predetermined linear one-way connection further, the oscillation between memories of the novel and the film is not hierarchical from a cognitive point of view, Hutcheon and Elliott (Rethinking) argue. Instead, they exist side by side, or like mirroring looking glasses (Elliott, Retbinking 222). Some people even read and watch in tandem, and perhaps also listen to the audio book, with multiple actors performing the individual characters and the narrator as a complement, not to mention the published screenplays, perhaps with photographs from the films and the film location, excerpts from novels, and the audio commentaries and storyboards that might be available on the DVD or BLURAY editions. This was indeed the case for me when I first got acquainted with the titles in the article sections of this dissertation. With Jenkins' text in mind, there is no reason to limit the possibilities of parallel experiences, when games, fan fiction, collaborative online research projects, speculations, book covers, and so forth may add content and value to the reading and watching.

In accordance with these trends, which are essentially ideological in that they favour diversity and non-hierarchical systems, in the intertextual approach to the study of adaptations it is preferable to place narratives side by side to observe the connections, rather than the actual objects, as Stam maintains (27). Sanders observes that "the web of intertexuality ... resists easy linear structures and straightforward one-to-one and one-way readings of 'influence"' (209). In scholarly practice, the linear one-way perspective is often assumed, however, even if network based theories are embraced in theory. For instance, Brian McFarlane's aim is to offer a productive method for intertextual researchers, but he still names his book Novel to Film, instead of Novels and Films. Likewise, in many studies the purpose is to show how certain later films have been informed by earlier novels. Still, just like audience research, the

\footnotetext{
${ }^{2}$ In fact, the film and novel are indebted to Arthur C. Clarke's short story The Sentinel (1948).
} 
intertextual approach opens up the possibility that newer texts may inform older ones, because they suggest, through intertextual play, what Sanders refers to as a "revised point of view" for understanding the works of fiction, which might also make older novels and films relevant for new audiences (18-19). As Morris Beja posits, "what a film takes from a book matters; but so does what it brings to the book" (88). From an intertextual perspective, it is thus just as relevant to ask how the narrative elements in Me Before You are used by Shakespeare, as it is to analyse how Romeo and Juliet inspires Jojo Moyes.

With this line of reasoning, MacDonald's aforementioned notion of the screen idea also relates to the work of novelists. Tom Perrotta, who wrote the novel Little Children and was nominated for an Oscar for the adapted screenplay, testifies that writing for the screen affected him as a novelist. Others, like Ian McEwan and Cormac McCarthy, have had several novels adapted for the screen, written screenplays, and acted as associate film producers. Dan Brown and J. K. Rowling signed contracts for adaptations of their novels before they had written them, and it is hard to imagine how these authors could not have been aware of a screen idea, with the unproduced films thus affecting the novels in a two-way exchange.

Jørgen Bruhn advances the idea of a two-way approach in adaptation studies by suggesting a dialogue between objects on the one hand and between objects and the adaptational processes on the other. He recommends the study of "both the source and result of the adaptation as two texts, infinitely changing positions, taking turns being sources for each other in the ongoing work of the reception in the adaptational process" (73). This holistic approach invites an understanding of a work of fiction as a "fluid text" and as "the sum of its versions," rather than as a single novel, screenplay, or film with a specific author attached to it (Bryant 47). A similar stance, which I have previously referred to, is taken by Cardwell, who considers the notion of a mythical or imaginary ur-text, which every adaptation in a network is a representation of, from different perspectives. Together they form a phenomenographic depiction of the ur-text and what it signifies. This allows a greater 
variation of titles to be studied in the same context, in order to examine a specific phenomenon. "The ur-text, if neither definite nor finite, if only postulated or implied, extracted from each telling of the story, cannot hold the same sway over adaptations (or retellings) that the standard whole does," she declares (26). With this model, the always-incomplete image of the ur-text replaces the source text, not, however, as the starting point for the adaptation process, but always as the result of an analysis of the elements that the stories included add to the picture. As Regina Schober submits, adaptations can thereby be studied as rhizomatic networks that reflect "multiple contextual entanglements" (118). Due to the polyphony of voices that such an approach may give rise to, the multiplicity of thematic implications and the multiple relationships between them may be impractical to study, unless they are limited through the use of simplifying and accentuating semantic structures.

These strategies for thinking about adaptations effectively deprive the fidelity issue of any relevance, but more importantly they abandon all hierarchical structures, so that every version of a story can be both at the centre of attention while allowing every other version to be so too, since their contributions are regarded as equal in relation to the whole. Moreover, they endorse the constructive position taken by Whelehan, who opines that adaptation studies is better served if scholars look for the "excess rather than lack" when the meaning of a story is considered (16).

\section{Fictional Beings in Non-linear Worlds}

As I indicated in the introduction, fictional characters can be regarded as fictional beings in fictional worlds. This still leaves us with the question of how the fictional worlds that they inhabit are constituted. For instance, we readily accept that Clark Kent and Lois Lane are the same fictional persons in a unified story universe, although they remain the same age regardless of whether they appear in stories set in the 1940s or in the 21 st century, so the time and place continua of our social reality do not necessarily seem to apply in the fictional worlds. Fans argue about whether Pride, Prejudice, and Zombies, with its altered universe, adds something of value to our 
knowledge of the characters, or if it should be regarded as an ironic tale, or a story about characters in a universe distinct from the one Jane Austen created and told her readers about. In these universes, characters may or may not have experiences and characteristics that are not shared with the audiences. For adaptations, that question is crucial because it concerns the possibility that fictional characters can migrate, appear, or even be reborn in new settings and narrative environments. With the different approaches to adaptations that I have discussed above in mind, I would simplify by suggesting that there are four fundamental ways of making sense of the stories about the characters in fictional adaptations.

First, the different stories may be seen as accounts about unrelated characters, who just happen to share certain properties, like names, addresses, and life paths, but nevertheless reside in different story universes which cannot be the same unless, perhaps, they share an authorial control. Second, the first or most canonical narrative of the characters' lives could be regarded as the true version, and all versions that digress from the facts in this version could be seen as narratives of false alternative facts, useful intellectual and imaginative exercises perhaps, but unfaithful to the characters' true destinies, to follow Umberto Eco's line of reasoning. Third, they may be regarded as complementary narratives, which agrees with the above-mentioned models of adaptations as fluid texts, or as parts of a network of versions. Each version or story that the characters are part of can thus be regarded as an account of their lives, and it is up to the members of the audience to construct a sensible whole out of these narratives, just as we do in real life when different versions of an event or aspects of a person are conveyed to us. In this context, Maria E. Reicher distinguishes between "maximal characters" as characters who have all the conventional properties of a certain character, and "sub-maximal characters," who have subsets of those properties (129). It is possible for those characters to be reborn, or regenerated, in new times and places, with properties that they did not have when and where they last appeared. Fourth, I would like to expand a bit further on Eco's discussion about migrating characters and entertain the idea of them as actual 
migrants who gradually transform their self-identities under migratory circumstances. Eco writes that characters may "leave the text that gave them birth and migrate to a zone in the universe we find very difficult to delimit. Narrative characters migrate, when they are lucky, from text to text" and between mediums (8). Thus, they "become individuals with a life apart from their original scores" (9), he argues. Other studies have made use of this metaphor, but what I propose is that we may better understand characters as fictional beings if we follow their migratory development, from novel, through a number of screenplay versions, to the screen, and so forth, and empathetically regard them as migrants.

Although the migrant analogy is commonplace in adaptation studies, it has become a politically sensitive metaphor. In everyday uses the term does not discriminate between suffering refugees and privileged, professional global workers who explore the world in their capacity as migrants. Originally, the term simply signified plants, animals, and people who change habitats, and in this context the word migrant is apt also for describing characters who move from one media form to another. The use of the term thus draws our focus to the phenomenon of changes in habitat or life environment and refers to the process of transition and adaptation to that new environment. Moreover, as other branches of science have done before, the migration metaphor invites new, productive theoretical models from migration studies to open up the study of media adaptations to include new perspectives.

When characters are considered as migrants, the bi-directional aspect of adaptations comes forth naturally in two ways. To begin with, if characters migrate they respond to their new environments and they change, but the acculturation process is always to some degree reciprocal, so that the culture of the host world also changes as a result of the new inhabitants' presence. As Bortolotti and Hutcheon puts it, "in a cultural context, adaptations influence culture and culture influences the nature of adaptations" (453). To study such migratory processes, from novel, through a select number of screenplays, to the screen, is not always possible, because the screenplays may not be available. In other cases, as I have described, the number 
of drafts and versions may be overwhelming, unless the observation points during the process are strategically restricted by the researcher.

To understand characters' development through a migratory perspective naturally demands another cross-disciplinary input. Based on the idea of a standard case, many studies and practices of managing migrants' development, when they adjust to a new society, rely on similar models of progression through specific stages (Stella Ting-Toomey and Leeva C. Chung; Ward et al.). These models are based on studies of migrants' integration into societies and are, of course, schematic and should not be taken to be valid for every individual case. Instead they describe ideal processes, based on averages. Nevertheless, what is most important is that the nature of the stages in these sociological models indicates productive parallels with models for problem solving, story arcs, and personal development, which can be productive for adaptation scholars. In the first step, a so called honeymoon phase, the migrant may embrace the new setting with naïve curiosity, and with little focus on internal change. In a second phase, the migrant experiences various kinds of conflicts, with negative consequences for their self-esteem, and with social withdrawal or anger as an effect. However, the standard migrant begins to approach the problem and sorts out the impressions and elements that condition the experience of the new world in a more constructive manner, in order to relate them to the former home culture and make a first attempt to change and to fit in. In the fourth step, the migrant experiences a sensation of being in sync with the environment, and can settle in and make judgements based on an understanding of the new culture, with the ambition of being able to function there as a citizen. At this stage, the fear of deculturalisation, or of losing any sense of self-identity if the changes result in exclusion from both the new and old culture, is common. Those who are often considered as successful migrants, however, tak a step towards a final reorientation so as to manage the core cultural and personal issues that may prevent their harmonious acculturation and resocialisation. When the characters have completed their journey and hopefully realised their self-identity in the world of the film narrative, they still carry traces and 
memories of their former selves and the places where they have resided before. As fictional beings they can never return to the novel without acknowledgment of their lives on the screen, because the characters, as Salman Rushdie recognises that he is, are "translated men" and women (17), with only "imaginary homelands" (10). In the end, the migrant can thus, ideally and with little effort, switch between self-identities and cultures, merge the past, present, and future images of the self into one, or choose one of the many experienced versions of the self to settle into and interact with the new culture in a relationship of acceptance. For both the migrant and for adaptation characters, self-identity can then, possibly, be found in a state of entropy, being one and many at the same time, as representations of the human condition in a world of flux. Where other methodologies fail to capture the process and object of adaptation simultaneously, a migratory approach does so by necessity.

\section{Adaptation and Appropriation}

Besides the word migration, adaptation studies and migration studies share terms like assimilation, accommodation, acculturation, adaptation, and appropriation. By definition these phenomena all involve transformative modifications of an object so that it may fit new purposes or environments. Assimilation signifies the process of adopting a culture or pattern to blend into. This would occur if a character from a novel is transformed to match a film stereotype. Accommodation, on the other hand, requires a flexibility and willingness, or ability, to change without vital sacrifices for the purpose of satisfying the new environment. Notwithstanding the relevance of these phenomena in this context, especially with the migratory, intertextual, and evolutionary perspectives in mind, in the last pages of this chapter I will focus on adaptation and appropriation, sometimes described as siblings in the context of media adaptations (Nicklas and Lindner 4). However, what makes them stand out is that they illustrate the contrasting bi-directional dynamics of adaptations.

Since adaptation has been adopted as an overarching term in media studies, it is necessary to clarify that, as processes, adaptation and appropriation are two 
different, albeit not easily defined phenomena within the field of adaptation studies. Many, like Sanders and Hutcheon, define appropriation more or less as a subcategory of adaptation, and Cartmell and Whelehan opine that every adaptation includes a purposeful use of appropriation (18), while Andrew merges the two concepts when he declares that "adaptation is the appropriation of a meaning from a prior text" (97). Still, as Sanders maintains, the two essentially denote different attitudes to and processes of change.

Adaptation, as a production process, takes its stance from a source, which is adjusted and developed to survive and fit in a new environment. From an identity perspective, if a character or story adapts to a new media environment, a core of that original identity will always remain, as I have previously indicated. Therefore, an adaptation does not necessarily mean that a character or story alters its content and meaning, apart from the connotations that follow with the new mode of representation (Elliott 133; Hutcheon 10). Adaptation thereby acknowledges the source as its principal subject, but adaptation is also subversive and oppositional, as Sanders argues, because it entails a break with a past tradition and a "rupturing of its value-system and hierarchies" (1). Moreover, if characters are regarded as fictional beings who migrate to new media environments, they are, within the fictional universe, equipped with a fictional agency, which makes it possible for them to adapt, as opposed to being adapted or forced to adapt, a circumstance which is closely related to many readers' and spectators' unreflected experiences of the characters' developments. For the creative team that adapts a novel, adaptation thus means a respectful negotiation with the story and the characters, with the adaptors functioning as counsellors in the adaptation process. With the same respectful attitude, building on Andrew's position that "every representational film adapts a prior conception" of media and genre conventions (97), the adaptors must also manage the new media environment's adaptation to the characters and story. Hence, novels, screenplays, and films, their genres and conventions are adapted to fit new 
stories and characters, just as stories and characters are adapted to suit new media environments.

Conversely, the pivotal agent in an appropriation process resides on the outside, usurping or taking "possession of another's story and filtering it, in a sense, through one's own sensibility, interest and talents," in Hutcheon's words (18). Sanders takes a step further and describes appropriation as a "decisive journey away from the informing source into a wholly new cultural product and domain," to the effect that "no appropriation can be achieved without impacting upon and altering in some way the text which inspired the adaptation" (158). Therefore, an appropriation does not necessarily flag the connection to any previous versions, since it can more or less be regarded as a "hostile takeover" from the fictional being's perspective (Sanders 9). Yet again, the relationship is bi-directional, so a fictional being may very well, with the help of a creative team, appropriate a media environment for its own purposes, with the purpose of surviving, remaining relevant, and reaching new audiences.

Given these definitions, it is still hard to conceive of any novel-film adaptations that do not include both adaptation and appropriation. No stories are ever either wholly adapted or appropriated, and no characters, stories, or themes are affected by one of these processes alone. By necessity, the processes of adaptation and appropriation are always at work in tandem. For the character, as a fictional being who migrates from novel, to screenplay, to film, this means that it adapts to the new media settings, while it is also appropriated by that new environment's culture and creative forces. The character likewise appropriates the new environment, which in turn adapts. Following from that line of reasoning, an adapted novel, with its thematic content, adapts to and appropriates the cinematic environment, while it is itself appropriated by the film culture, which also has to adapt. 


\section{Chapter 3}

\section{Characters' Thematic Functions in a Narrative}

In this chapter, I first discuss the thematic functions that characters have in narratives. I then present a model of how emplotment frames thematic arguments in fiction with a rhetorical structure, dispositio in Aristotelian terms, based on the progression of the protagonist's journey through a narrative, as it is outlined by Joseph Campbell in The Hero with a Thousand Faces (2008). Thus, I argue for its use as a pragmatic approach to thematic adaptation studies. The latter part of the chapter is dedicated to the example theme, adulthood. After a brief overview of aspects that have been connected with adulthood through the study of how the theme is represented in fiction, developmental psychology, and sociology, I introduce a set of thematic markers of adulthood that can be employed together with the plot model to detect thematic aspects and functions in a narrative.

\section{Characters and Thematics}

The crucial role of characters when readers and spectators engage in fiction was indicated in chapter 1 . As Jens Eder et al. proposes, characters "are devices in the communication of meaning and serve purposes beyond the fictional world as well" (45). In accordance with this, Booth observes that "we look for an answer to the question, "What do these lives mean?" and for "complete patterns of theme," since works of fiction commonly "make us desire a quality," such as truth or knowledge (Rhetoric 126). Keys to the insight that readers and spectators are searching for may be accessed through an engagement with a fictional narrative, but the actual insights are usually related to readers and spectators themselves and to the real world. Margaret Mackey has noted that some readers enter the story world mentally and use internal inferences to orientate themselves in that world, whereas others evaluate its actual presentation from an external perspective. Some mainly assess their reading through thematic approaches, and some primarily engage their knowledge of genre conventions and their textual expectations to orient themselves in the narrative (102). 
The reader can thus choose to move, or be moved, between levels of engagement (Gerrig), and to alternate between internal and external perspectives (Currie; Walton). Likewise, as Rick Altman suggests, fictional narratives are commonly designed so that readers and spectators can see matters from a character's point of view. This inher-shoes imagination, or the simulation of being a character, enables us to sense what the characters feel and think and to incorporate those experiences into our own systems (Currie 108), although the characters do not exist in the corporeal world.

However, due to their ontological complexity, the terms of the existence of fictional characters, their constitution, and how they can be understood are highly debated issues. Steven Price notes that some strands of traditional literary and film criticism regard fictional characters as pure textual, semiotic constructs, and claim that all that can be established about them "is what is present on the page," whereas, for instance, screenwriting manuals often describe characters as real human beings, with lives outside the texts ("Character" 202). Shlomith Rimmon-Kenan outlines the conundrum that characters present as parts of texts: they are designs, as well as possible beings in fictional worlds.

In the text characters are nodes in the verbal design; in the story they are - by definition - non (or pre-) verbal abstractions, constructs. Although these constructs are by no means human beings in the literal sense of the word, they are extracted from their textuality. Similarly, in the text, characters are inextricable from the rest of the design, whereas in the story they are extracted from their textuality. (35)

One reason that textual representations of characters, whether bound by the constraints of the conventions of novels or films, often invite such extractions is that they can be presented with abundant attributes. Mieke Bal suggests that it is the surplus of information, "the piling up of data" (126), which thus facilitates the conception of characters as plausible fictional beings. This can be likened to the process of gradually getting to know a person in real life, but unlike people in real life, fictional characters rely on a creator's characterisation to exist. Characterisation 
is, simply put, the attribution of qualities to a character, which in a broader sense includes all the information that can be associated with a specific fictional character (Eder et al. 31). Some theorists, like screenwriting specialist Robert McKee, distinguish between character, the inner personality of a fictional being, and the characterisation of what can be observed from the outside. Uri Margolin refines this rough model usefully and proposes that four different layers of characterising attributes, or qualities, should be distinguished: static facts, dynamic expressive acts, habits, and traits. First, static facts refer to discrete details like name, age, appearance, and where a character normally lives. Second, when a character is reported to think, speak, feel, do something, or appear in a specific environment, they perform "verbal, mental, and physical acts" which tell us something about the character's personality (206). These dynamic expressive acts gradually add up to systems of consistencies and inconsistencies, and may thereby make evident what is normal or habitual for a character. Thus, habits, the third layer, can be defined as repeated patterns of physical, social, or mental actions. Fourth, it is possible to attribute fairly consistent personal qualities, or traits, to a character through his or her expressive acts and habits. ${ }^{3}$ As each piece of information constitutes part of the frame for the next, the attributes gradually accumulate during the progression of a narrative and make it possible to generalise, classify, and interrelate the details about a character. In this process, all forms of relative emphasis on attributes in the narratives, such as repetitions, contrasts, durations, relationships to other characters, and potential changes in a character interrelate so that a reasonably full portrait of an as-if real person (Margolin 206), or, in Phelan's words, the "illusion of a plausible person" (11), takes shape.

Still, the mere piling-up of information is not enough for more than a fixed portrait. The above-mentioned aspects might be said to constitute the foundations for a character's agency, which writers and directors seem to bestow them with, to

\footnotetext{
${ }^{3}$ See Seymour Chatman's Story and Discourse, $121 \mathrm{pp}$. for a discussion of traits and habits.
} 
make them appear "to have existence beyond the limits of the text" through the audience's imagination (Price, "Character" 203). Characters thus appear to have "corporeality, mind, and sociality" (Eder 24), with "goals, intentions, emotional responses, relations, contacts, habits, social patterns, in-groups” (18). Joseph Carroll exemplifies this fictional agency by describing how characters sometimes "struggle with one another to tell the story in their own terms, and the author constantly mediates among characters, aligning himself or herself with one or another" (Literary 150). Likewise, David Wiegand declares, about the characters in Ian McEwan's Atonement, that "unlike fate-doomed characters in 19th century novels, the Tallises have the power to make different choices - if only they would take responsibility for their actions." Although Joseph Carroll and Kermode do not believe that fictional characters actually possess the individual agency and personality of real world individuals, they acknowledge that most readers and movie-goers temporarily imagine that fictional characters have the same privileges as corporeal social beings in that respect. As Eder consequently declares, "we perceive characters as thinking, feeling, and active beings is in many respects the most important aspect of their reception" (Understanding 23).

Yet, regardless of the medium, fictional characters neither leave the story world entirely, nor do they take a corporeal shape of their own, which is not tied to the creation, conditions, and existence of that world. This is valid also for films and stage plays, in which characters assume the appearances of actors from the real world. However, they can still be described and taken seriously, as if a non-existent person could possess agency and a personality, or an individual character (Bourbon 73). The reason for this is that they are created with a communicative and thematic purpose, James Phelan argues, and he proclaims that characters' "creators are likely to be doing something more than increasing the population, more than trying to bring plausible persons into the world" (Reading People 14). If characters were merely designed fictional beings, without thematic content, they would only randomly communicate anything of value to the audiences, and their design qualities would be of a mere 
decorative value. Instead, the mimetic and synthetic qualities of characters are there to promote and enhance the viewer's or reader's attention to specific attributes and motifs, ${ }^{4}$ and to their symbolic (Eder), and thematic dimensions (Phelan Reading People; Tomashevsky 68). Boris Tomashevsky even articulates that a "protagonist is by no means an essential part of the story," because their foremost function is to embody and demonstrate the thematic idea (90), to the effect that every character personifies complementary ideas and contributes pieces of symbolic meaning to form the narrative argument around a theme. As Eder et al. posits, "characters fill positions and roles in larger webs of relations, such as structures of communication and narration; constellations of characters; situations, plots, themes; and effects of an aesthetic emotional, rhetorical or ideological kind" (45).

Broadly speaking, the design of characters' thematic dimensions thus relates to the actual narration and involves all the textual or cinematic manifestations of choices and decisions by a writer, or by members of a film team who work together to present the characters' attributes. Most importantly, the design cues the reader's or spectator's attention so that it is directed towards the relevant pieces of information about characters, and therefore also guides attitudes and interpretations, as Bordwell argues when he calls for a consideration of characters and narrative functions as designs ("Neo-structuralist" 204). Thus, it is not enough to study how characters appear as fictional beings in the context of their fictional worlds, or how they are constructed, to fully understand what they may represent in a work of fiction. Eder suggests that one should first examine the features of the fictional being, then its construction as an artefact, and subsequently the relationships between characters, actions, and character constellations, as a foundation for an investigation of characters as symbols and symptoms of phenomena in the real world.

\footnotetext{
${ }^{4}$ Boris Tomashevsky defines motif in Thematics as "the theme of an irreducible part of a work" (67), referring to a statement sentence which can be ascribed to a single event or attribute, before he discusses the difference between this definition and motifs as elements in comparative studies (67-8).
} 
Still, it is only when the narrative emplotments charge and activate characters and their attributes that their potential as semiotic resources in elaborate thematic lines of reasoning are realised and can come to a conclusion with the narrative ending (Tomashevsky 63). The orchestration of the thematic development through the plot gives the characters' attributes their thematic functions, Phelan reasons, and he outlines, in line with Tomashevsky, how the organisation of character attributes and thematic functions forms arguments which are gradually developed through the plot of the narrative. As the plot evolves, so does the thematic case. In effect, the thematic significances which a character and a character's attributes carry depend on the way they are foregrounded in and developed in relation to the plot (Brooks; Phelan; Hogan).

Among others, Vogler, McKee, Craig Batty, and Syd Field, writing about screenplays, describe how characters are part of a rhetorical structure and serve the purpose of demonstrating the controlling idea or theme, mainly through dramatic conflicts. Likewise, Lajos Egri outlines how the thematic idea in a drama is advanced through an orchestration of "well defined and uncompromising characters in opposition, moving from one pole toward another through conflict" (119), based on dialectics and the idea that problems and crises produce development. From a dialogical perspective, however, characters and their attributes need not be arranged as opposites, but should rather be considered as complementary parts of a metaphorical reasoning around a theme, adding nuances and counter-ideas of various weights in a narrative. Just as characters' individual attributes are distributed and orchestrated to form a greater whole, the plenitude of characters and attributes in a work of fiction creates a polyphony of aspects of a theme "and, as such, are combined in a unity of a higher order" (Bakhtin, Problems 21).

A plot structure, I argue, partly functions as a structuring axis or instrument with which to disentangle the polyphonic orchestration of characters and their thematic functions. To identify thematic attributes is simply to ask whether a character or a situation involves a certain quality, or not. In that way, Bal explains, 
"mutual relations between the characters are immediately visible," and complex patterns can "be mapped out" (129). More importantly from a rhetorical point of view, the specific expressions, contextualising normalities, instabilities, developments, and resolutions play important roles in the accentuation of thematic nuances in the plotted argument (Phelan, Reading 15). Similarly, from a semiotic perspective, Gerard Genette defines order, frequency, duration, mood, and voice as a core of synthetic aspects that also contribute to the articulation of the rhetorical communication of meaning in a narrative. Thus, any simplistic semantic gradation must be complemented with a richer thematic analysis of attributes, instances, and functions in relation to the thematic progression through the plot, character context, and not least in light of how the information about a character is conveyed in the narrative.

\section{The Rhetorical Emplotment of Themes}

As suggested, characters' thematic functions in a narrative are essentially organised by means of the plot. In other words, the design of thematic attributes and events in a specific order provides the narrative its rhetorical logic. Hogan identifies three universal plot prototypes, based on romantic, heroic, and sacrificial themes, and there are obviously numerous strategies to modify the prototypical plots, and certain dramatic narrative elements, functions, and underlying ideologies can be used to define and create subgenres. On the other hand, as Bordwell argues, all genres, including complex multi-narrative films, share a "hyper-classical" plot, in which every part of a narrative conforms to a pattern and thus adds to the overall discussion of the controlling idea of the work (The Way 62). Following this line of reasoning, the study of thematic representation through characters becomes partly a matter of studying classical plot structures, and the thematic development through the characters' journey through a narrative. As described above, throughout the progression of the plot, the characters' attributes thus fill thematic functions to 
explore the themes of the narrative rhetorically, test their constitution, render clarity, and reach a thematic conclusion.

To study the thematic emplotment in novels, screenplays, and films side by side, and do them justice, demands a model which is fairly impartial to the medium. Based on an Aristotelian stance, creative writing manuals for screenwriting and novels commonly use similar plot models, and theories of literature, screenplay, and film share that foundation. As is often the case, the differences appear more clearly within a specific field than when two fields are compared. However, at this point, I refrain from an overview of different plot theories, and only record that the threeact structure is still the dominant model, dividing narratives into an exposition, a complication, and a concluding denouement. Yet, as Thompson, for instance, has wisely suggested, if the second act, which is often described as having the same length or duration as the other acts put together, is split into two, then four equally long parts with distinct functions can be conceived: a setup, a complication, a development, and a resolution or conclusion (22). From a thematic point of view the first act could be understood as the presentation of a theme. This theme is complicated in the second, developed towards a new understanding in the third, before the concluding fourth act.

It is in this context that Campbell's notion of the monomyth can be of instrumental value. His theory regarding the universal thematic features and structures of myths has been adapted and popularised in numerous creative writing manuals and narrative models (see Vogler's The Writer's Journey and Batty's Movies That Move Us). It has been applied in the creation of feature films and novels alike, most famously George Lucas' Star Wars saga, which has given the hero's journey a mythic status of its own among screenwriters and film producers. This does not mean that the hero's journey is less applicable to novels. Essentially, Campbell extracts a narrative model of the hero's journey in seventeen developmental steps, wirh specific functions, towards a mythical "adult realization" (12), and a possible full "reintegration with society" as a responsible person (29), which makes it particularly 
relevant when the representation of adulthood is studied (see table 2). The seventeen steps are also presented in a circular model with four functional quarters, although he divides them into three sections in the text. Due to Campbell's Jungian stance, Maria Nikolajeva submits that, although this narrative structure can be traced in most narratives, it is nevertheless "not uncontroversial in its overtly psychoanalytical orientation and must, therefore, be applied with some caution" (31). Unfortunately, instead of adhering to that advice, many applications of the hero's journey instead simplify and reduce Campbell's writings so considerably that all that remains are a few steps, a causal chain of events, and individual character development in relation to a specific problem. Batty and Vogler both reduce the middle parts of the narrative to a few steps, although their models have other benefits. John Truby's The Anatomy of Story connects the plot to the moral argument of a story. However, I find his suggestion that a story is broken down into twenty-two steps, with its emphasis on external events, even more convoluted in its thematic logic than Campbell's seventeen step structure. What many narrative models thus ignore is Campbell's focus on the human condition in the social world at large and the opportunity to consider the hero's journey as a comprehensive, rhetorical presentation of thematic content.

Because each step in the hero's journey has a specific developmental function, forming part of a causal chain, the narrative structure can be translated into the structure of a thematic argument (Hermansson, Towards 2020). Campbell sometimes uses an abstract and today dated terminology, based on various common elements that he has found in myths, and Batty ("Physical") and Vogler have both suggested productive modernisations of the terminology that indicate the functions of the various steps more clearly. They both focus on the inner development of the protagonists, their inner progression, and the plotted events, the physical journeys, in the narratives, but with headings like "limited awareness" (Batty, "Physical 300), "return with elixir," and "final mastery of the problem" (304), their terminology invites an adaptation of the hero's journey into a narrative guideline for a rhetorical 
thematic line of argument. As suggested, I also find a variation of Thompson's four act structure and the terminology she uses helpful in a rhetorical context: Introduction/Setup, Complication, Development, Conclusion.

Converted to a structure for a thematic line of argument or journey, the narrative structure could thus read as follows (see table 1 below). In the first act's presentation of a theme, Introduction/Setup, the initial step is to depict an unreflected upon, everyday understanding of that theme, based on a limited awareness and a narrow "familiar life horizon" (Campbell 43). Then follows a call to explore the theme, and a first argument for a less simplistic approach to it, perhaps through a demonstration of the shortcomings of the current conception. A counter argument in support of the initial understanding of the theme ensues, which demonstrates the strengths of the habitual thematic notion. Next, a mentoring phase emphasises what there is to gain from a developed thematic idea and points out the issues of concern again. The first act ends with a crossing of the threshold to investigate the theme further, which can be likened to "a form of self-annihilation" (Campbell 77), since the old views are left behind, and the formulation of a new definition begins with as little as possible pre-defined.

The second act's rhetorical purpose, according to Thompson, is to reveal and address the complications, as indicated by the label Complication (see table 1, column 2). In Campbell's terms, it comprises the first half of an "initiation" to an evolved thematic idea (81). It begins with an inventory and organisation of new and old aspects, and the pros and cons of their contribution to the thematic idea. Initially, Campbell thus advises a dissection of the theme into dialectic pairs, to lay bare the complications, and a vision of the "totality of what can be known" (97), through a "harmonization of all the pairs of opposites" (95). This leads to a provisional summary and a temptation or question regarding whether to settle, to abandon the exploration, or to continue. Hence, at the midpoint of a model narrative, the current "position of reference" to the "general ... formula" (101) is tested and stock is taken of the remaining results and problems. 
In the third act, the Development of the narrative's argument (see table 1, column 2), the still unresolved core issues of the thematic understanding are confronted, in order to form a developed notion of the theme, which makes allowances for less idealistic aspects to be included. Commonly, some previous underlying presuppositions or convictions have to give way or be redefined. With these problems settled, the new thematic idea can be formed. The final stage of the third act entails a summary of a tentative new, theoretical or abstract, thematic comprehension as an "indestructible body" (150).

The fourth act, Conclusion (see table 1, column 2), begins with "obstruction and evasion" (170), as Campbell posits, because what remains before the conclusion is to demonstrate how the new thematic insights may be applied in real life. The first step thus confronts the doubt that they can be put into practice. The next step demonstrates how they can be applied in typical situations, for instance to approach and overcome practical problems, or how they can be integrated with other ideas. For a thoroughly convincing and complete argument, the practicability of the thematic idea is then proven under more authentic testing conditions, which demands certain compromises and adjustments, not least the management of the consequences. Finally, "the elixir" (Campbell 170; Batty, "Physical," 304), or solution and formula of the new thematic understanding, and a vision of future possibilities, if it applies, is presented.

As implied at the beginning of this outline, it can be argued that Campbell's structure, with its emphasis on the development of the relationship between the ego and the communal, affects the observations of how the markers of some themes, especially regarding a theme like adulthood, are distributed in a biased way. The obvious link between the hero's journey and growth, from limited awareness to maturity to developmental theories, may also add relevance when adulthood, for instance, is studied as a theme in narratives. 


\section{Table 1}

The hero's journey transformed into the structure of a thematic line of argument.

\begin{tabular}{|c|c|}
\hline $\begin{array}{l}\text { The hero's journey: Joseph Campbell's } \\
\text { / Craig Batty's terminology }{ }^{5}\end{array}$ & $\begin{array}{l}\text { The hero's journey as a thematic line of } \\
\text { argument }\end{array}$ \\
\hline Departure & Act 1: Introduction/Setup \\
\hline $\begin{array}{l}\text { World of common days / Ordinary world, } \\
\text { limited awareness of a problem }\end{array}$ & The ordinary understanding of the theme \\
\hline $\begin{array}{l}\text { The call to adventure / The call to adventure, } \\
\text { increased awareness }\end{array}$ & A call to explore, since there is more to understand \\
\hline $\begin{array}{l}\text { Refusal of the call / Refusal of the call, } \\
\text { reluctance to change }\end{array}$ & A counter argument to stay with the initial understanding \\
\hline $\begin{array}{l}\text { Supernatural aid / Meeting the mentor, } \\
\text { overcoming reluctance }\end{array}$ & Benefits with development of the thematic understanding \\
\hline $\begin{array}{l}\text { Crossing the first threshold / Crossing the } \\
\text { first threshold, committing to change }\end{array}$ & Crossing the threshold to investigate the theme further \\
\hline Belly of the Whale / n. a. & Beginning with a clean slate \\
\hline Initiation & Act 2: Complication \\
\hline $\begin{array}{l}\text { The Road of Trials / Tests, allies, enemies, } \\
\text { experimenting with first change }\end{array}$ & Known and new elements are sorted out \\
\hline $\begin{array}{l}\text { The Meeting with the Goddess / n. a. } \\
\text { The Woman As Temptress / n. a. }\end{array}$ & $\begin{array}{l}\text { New aspects, complications and potential are summarised } \\
\text { Evaluation of potential in development }\end{array}$ \\
\hline & Act 3: Development \\
\hline $\begin{array}{l}\text { Atonement with the Father / Approach to } \\
\text { the inmost cave, preparing for big change }\end{array}$ & Confronting the core of the previous conception \\
\hline Apotheosis/Ordeal, attempting big change & A whole new perspective \\
\hline $\begin{array}{l}\text { The Ultimate Boon/Reward, consequences of } \\
\text { the attempt }\end{array}$ & A tentative new definition \\
\hline Return & Act 4: Conclusion \\
\hline Refusal of the Return / n. a. & Doubts before practice \\
\hline The Magic Flight \& Rescue from & Examples of practical application and integration of the \\
\hline $\begin{array}{l}\text { Without }{ }^{6} \text { / The Road back, rededication to } \\
\text { change }\end{array}$ & thematic understanding in real life \\
\hline The Crossing of the Return & Confrontation with remaining practical complications and \\
\hline $\begin{array}{l}\text { Threshold/Resurrection, final attempt at big } \\
\text { change }\end{array}$ & compromises \\
\hline $\begin{array}{l}\text { Master of Two Worlds/Return with elixir, } \\
\text { final mastery of the problem }\end{array}$ & Conclusion and new thematic understanding \\
\hline Freedom to Live / n. a. & The happiness that a developed thematic concept offers \\
\hline
\end{tabular}

Source: Campbell (2008); Batty (2010); Hermansson (“Adaptations of Adulthood” 2020)

\footnotetext{
${ }^{5}$ Batty groups several of Campbell's steps into one, hence leaving some of them out.

${ }^{6}$ Campbell describes these two steps as two variations with similar functions, and suggests that one or the other, rather than both, often occur in stories.
} 


\section{Adulthood in Fiction}

In Visions of Virtue in Popular Film (1999), Joseph Kupfer examines the representation in mainstream films of what it is to lead a good life as a mature person, and of the qualities that make people valuable, both to themselves and to others, in those films. Based on Aristotelian ethics, he focuses on the demonstration of "settled dispositions to act, feel, desire, and think in specifiable patterns" (25), "with enduring character traits" (31). First and foremost, Kupfer detects the importance of selfknowledge, realistic perspectives and optimism, courage, the ability to improve, practice, perseverance, practical wisdom, and playfulness in the films analysed. Intimacy, community through love, and friendship are other prominent, substantive virtues on film, Kupfer suggests. Equally, qualities like self-deception, conceit, impatience, egoism, desire, and greed are observed as typical character traits that characters must learn to keep in balance through the narratives. His conclusion is that mainstream films seem to "stress the social nature of human existence" (228) and that "the goodness of life ... is found in activity: in the moral doing, the artistic making, the rational thinking" (37). The qualities he observes, I will argue below, are similar to the traits that are commonly associated with what it is to act as an adult.

Stories about the conditions for adulthood can be found under labels like the Bildungsroman, development and coming-of-age stories, crossover fiction, Young Adult (Y A) fiction, and most recently new adult $(\mathrm{N} A)$ fiction. ${ }^{7}$ Each generation of authors and critics seems to relabel the genre in relation to current ideologies and sociological conventions. Although they are epistemologically different, autobiographies and biographies could be attached to the same cluster of terms, since narratives in these genres normally share the general structure and central narrative features with fiction about the conditions for adulthood. As Kenneth Millard notes in Coming of Age in Contemporary

\footnotetext{
${ }^{7}$ The currently most popular term, Young Adult fiction, has probably been in use since at least 1802, when Sarah Trimmer referred to 14-21 year olds as "young adults" in The Guardian of Education. The American Library Association is also said to have coined the term Young Adult fiction in connection with their first list of Best Books for Young Adults in 1966. As the age of youth has gradually been extended, the term has come to refer to fiction about people up to 30 years of age. NA fiction was coined by St. Martin's Press in 2009 to market fiction about "older YA.”
} 
American Fiction (2007), "the protagonist ... commonly starts as a novice, encounters and overcomes the challenges of adversity and ends ... as a more mature adult character as a result of their experience" (3). Although he focuses on contemporary American coming-of-age novels, his reflection is valid in a wider context. In novels and films, the characters' processes of finding themselves and adapting to the conditions of adult life are associated with age-specific emotional experiences. Noticeably, Anita Wohlmann finds that ageing is mostly related to negative sensations in fiction, such as "worthlessness, inadequacy, shame, or anxiety" (252). She admits, though, that in Young Adult fiction, adulthood can also be linked to "hopeful messages of growth, trust, and togetherness" as well (252). This apparent contradiction, as Rachel Falconer observes, can be explained by the fact that fiction related to age usually hinges around themes that define various threshold states. The narratives commonly follow characters' "attempts to formulate a positive subject position" (Stewart 182), and their efforts to find "a sense of meaning, an identity, a truth" (Gillis 52) and keys "to be[ing] morally good" (Carroll, Minerva 101). In sum, as Wohlman argues, stories revolving around adulthood as a theme generally address the questions "Who am I?" and "Who could I become?" (88). Thus, the conventions of the Bildungsroman still noticeably govern fiction about adulthood and it will therefore figure as a recurring thread through the section below. Although studies of romantic comedies, chick lit, and the detective genre could well be included in the survey in the forthcoming pages, as could genre specific aspects of the theme, I will focus on the representation of adulthood in the genres that most clearly direct their attention to the theme.

In 2009, Melanie D. Koss and William H. Teale surveyed 370 titles of literary Young Adult fiction and found a thematically fairly well defined genre. For example, $85 \%$ of the characters in the study are occupied with "finding themselves" (567). However, there are specific contemporary trends. Koss and Teale noticed a contemporary shift from the traditional Bildungsroman and coming-of-age story formats to stories covering a shorter time span. This seems to suit the tendency to 
structure contemporary representations of the conditions of adulthood in mainstream fiction around specific dramatic topics, within predominantly monocultural social spheres. Hence, life-threatening situations, such as accidents or cancer, have been used as premises in many stories that thematically revolve around adulthood. 25\% of the protagonists, in Koss and Teale's study, suffer from diagnosed disabilities, mental or physical (566). Other scholars have identified homosexuality, drugs, gender, parenthood, ethnicity, technology, and materiality as frequent features in contemporary fiction about what it is to be an adult. A common thread is that characters struggle with combinations of loss, moving, bullying, friends, family, love, abuse, and illnesses in their attempts to find a stable subject position in life, before it is too late, in the face of the threat of social invisibility and non-existence.

The list of highlighted subjects and dilemmas indicates some of the social, mental, and ethical challenges that fictional beings encounter as they strive to become, or be, responsible, stable, reliable, and autonomous adults in their fictional worlds. Strikingly, the topics listed above also give the impression that fiction which thematises adulthood is dominated by crises and the darker sides of life. Nonetheless, it has been argued that the consequences of choices and actions are not adequately problematised in fiction about adulthood and that the genre thus glamorises the problems of life. Indeed, "the excitement of a forbidden underworld" (Nelms, Nelms, and Horton 92) and the fascination of alternative, carefree lifestyles seem to have a provocative and subversive ethical power on the surface. However, the representations of these ideals in fiction about adulthood seem to generate little general and lasting attraction. Although most of the stories question contemporary social ideals, adulthood is depicted as an aim in life: a desired state of normalcy and of reasonable contentment that is achieved through inclusion in society. ${ }^{8}$

Consequently, fiction that is mainly concerned with adulthood also revolves around representations of the social and political world, to follow Millard's argument.

\footnotetext{
${ }^{8}$ Science fictional stories also come to similar conclusions about being human in general (Ostry 243).
} 
Just as the classical Bildungsroman can be located in the context of the bourgeois ambition of ennoblement (Moretti viii), contemporary fiction about adulthood creates role models for middle-class life and identity, as Wohlmann suggests (46), or, more drastically, microcosms where individuals are curbed by social control to adapt to the dominating adult middle-class norms (Lopez-Ropero 155).

Yet, as Millard maintains, these contemporary narratives generally strive to contextualise the imbalance that the characters experience, between the demands of society and social life, their historical determination, and the idea of "self-fashioning" (10). His analysis thus indicates that the conventions of the Bildungsroman still carry weight in fiction about adulthood. For instance, as Franco Moretti demonstrates, the protagonists in the Bildungsroman tradition labour above all to come to terms with the conflicting values of being an individual and being a part of a greater social whole (27), which is also the central point in Joseph Campbell's version of the hero's journey, and in many contemporary films and novels. Sprung from the romantic ideals of individualism, the general goal of the classic Bildungsroman was to build the ego. Yet, the value of youthful individuality is clearly subordinated to the worth of maturity and inclusion, Moretti argues (27).

The aim of the protagonists of the Bildungsroman, Moretti thus claims, is not individual freedom, but rather "proper functioning" and socialisation within the scope of normality (11). The same ambition of social adaptation is still the permeating theme in contemporary high school or college movies, for instance. As Timothy Shary observes in Generation Multiplex: The Image of Youth in American Cinema since 1960 (2014), the jock, the nerd, the popular, the rebel, and the delinquent are part of an orchestration of various individual human ideals, but they all strive for inclusion and recognition. Seemingly, fiction about adulthood still follows the conventions of the Bildungsroman as far as quests for liberation, self-discovery, and social adaptation are concerned. In the end, the protagonists seek to accept and adapt to the conditions of the world, while growing into more mature individuals. 
Moreover, the protagonists in fictional stories with a focus on adulthood also demonstrate a capacity to listen with a sensitive intelligence, which enables them to adapt and to consider the well-being of others (Moretti 50-51; Shary 36; Carroll, Minerva 96). This is also true for Dan Brown's hero, Robert Langdon, in The Da Vinci Code. Shary notes that younger rebel protagonists, in particular, often also recognise and constructively take on the more serious social and political patterns of their story worlds, in order to be included in society as worthy adult persons (259). In effect, the prototypical characters in fiction concerned with adulthood demonstrate "that meaning $[\ldots]$ has its price. And this price is freedom," as Moretti suggests regarding the essence of the Bildungsroman genre (63).

As indicated, the representation of the conditions for adulthood in these narratives indicates that individual change and freedom are not the ultimate goals. Instead, these values must to some extent be sacrificed for the sake of security, community, and a sense of a fairly stable self-identity that adulthood has to offer. Thus compromise, Moretti finds, is the Bildungroman's "most celebrated theme" (9), because what is presented is not an idealised vision, but a humanising lesson about being an adaptive person in everyday life, with its trials and comforts (35). So, Moretti explains, "if the hero wishes to enjoy absolute freedom in a specific domain of his existence, in other sectors of social activity there must prevail instead complete conformity" (55).

It must still be noted that, as Millard and Wohlmann both observe, fictional representations of adulthood have traditionally been crafted from male centred perspectives, and it is only recently that gender awareness has steered academic and creative interest towards a productive focus on the female conditions for the transition to adulthood and to female adult lives. However, worries about professional careers, authority, and financial stability remain significant issues regardless of the characters' gender in fiction about adulthood (Moretti 255). Yet, in American coming-of-age fiction about female protagonists, the conflict between socialisation and inclusion on the one hand and freedom and individuality on the 
other is intensified, as Christy Rishoi notices. As a result, the female rebels often choose to remain as outsiders, and resist being subordinate to the conventional social structures of adult life, such as marriage, building a home, raising a family, and having permanent employment, since they are denied the same recognition that male characters gain within the community if they conform to these conventions. Hence, fiction about female protagonists who are struggling with adulthood often further emphasises the recurring topic of the abuse of social and political power as threats against the protagonists' autonomy within the system.

Similarly, as Aleid Fokkema submits, it is common in postmodern fiction for characters not to reach the insights that will give them permanent stability in adult life. As adults, they still struggle with the conflict between the public and private spheres, trying to control chaos in a world of power relations. Nevertheless, Fokkema maintains, the dramatisations of thematic problems related to adulthood in contemporary fiction illustrate the idea that being able to use experiences for practical purposes leads to recognition, as adults, from others. When the protagonists obtain wisdom and knowledge of the world, they learn to navigate through the conflicts and the problems of adult life with a sense of rationality, stability, and respect.

As argued above, the representation of adulthood is an issue of concern in most fiction. Hogan's analyses of world stories further highlight that characters generally relate to certain qualities in order to normalise happiness and meaning as adults in fiction. Health, attachment, and community, coming to terms with sacrifice and imperfection, balanced emotions, justice, and the ability to act to achieve goals are some of the dominant elements, Hogan suggests. This research overview also indicates a focus on social, adaptive qualities in films and novels that thematise adulthood. However, fiction about adulthood seems to insist that to reach the point at which a person can be regarded as an adult by those in their environment, the protagonists must compromise and adapt their social structures and patterns, individual agency, world-views, and virtues to the societal environment. In the end, 
individuation and socialisation are apparently equal parts of the prescribed image of adult personhood in fiction.

\section{Just Be Adult about It - Markers of Adulthood}

Although physical and neuro-cognitive changes gradually develop the human body and mind, adulthood is primarily a socially constructed and mediated concept of idealised normalities in real life, just as it is in novels and films. Legal regulations may deem people to be perfectly adult after a certain age, but it is impossible for any human being to live up to the standard of the adult ideal at all times without lapses into what could be labelled youthful behaviour. Conversely, children may temporarily show capacities that are associated with adult behaviour or thinking, without being adults, since adulthood is equated "with full personhood in terms of the recognition of actors as 'full partners in interaction'," to speak with Harry Blatterer ("Changing" 66). The notion of adulthood thus encompasses a vision of the fulfilment of a complete set of interdependent qualifiers, which I will refer to as markers of adulthood. Presently I will outline a tentative, but for this project purposeful, definition of adulthood as an ideal. Because adulthood is a societal ideal, being acknowledged as an adult ultimately depends on the social recognition of others, subject to one's performnces of stability, reliability, responsibility, and autonomy being evaluated as consistent (Blatterer, "Changing" 66; Molgat 498). To complicate this, instead of being defined by discrete variables, adulthood seems to be a matter of evolving a tolerant balance between competencies: an acquired ability to handle life, property, and community, a responsible and social adaptation to the social environment, and to the norms of society (Blatterer, "Changing"; Arnett Emerging).

Interestingly, studies of evolutionary aspects of human behaviour, of adulthood, and of ego formation all employ similar markers and components of reference for what may be conceived as ideal or conventional normalcies (see Joseph Carroll; Erikson; Marcia; Metzinger). In developmental psychology adulthood is regarded as a usually stable phase of life concerning personality, identity formation, 
and identity status. Erik H. Erikson distinguishes between the adult personal identity, which encompasses stable values, beliefs, and sets of goals, and the social identity, formed by the adaptation to group ideals and the capacity to blend into a community. In effect, Erikson's theories also suggest that a prototypical adult has established a sense of autonomy and integrity, and is capable of trust, intimacy, stable relationships, initiative, collaboration, and productivity. These elements bear resemblances to predictors of well-being, as described by Carol D. Ryff, such as balanced emotions and behaviour, self-acceptance, autonomy, purpose in life, stable and close relations with others, and an ability to adapt to the environment.

However, modern life patterns open up differentiated and destabilised pathways to adulthood, since the definition of adulthood is to some extent dependent on the socio-cultural environment. There is also a consensus that the transition period between adolescence and a well-defined state of adulthood is lasting longer and longer (Côté and Bynner 253; Arnett, Emerging 3). For some groups, education extends the period, for others, economic or social circumstances do. Nevertheless, when sociologists study life patterns, expectations of adulthood, and cultural associations with the concept, they find that the characteristics that define people's understanding of what it is to be an adult are fairly constant globally and over time (Blatterer, Coming 15). Some particulars may vary and depend on local, societal conditions, like having a car, but the qualities of which these are representative, such as independence, social adaptation, reliability, and competence, remain the same.

\section{Markers of Adulthood}

In the following, I present a model for grouping markers of adulthood that have been observed and discussed in a variety of articles and monographs, such as Erik H. Erikson's Identity and the Life Cycle (1980), Lewis R. Aiken's Human Development in Adulthood (1998), James A. Côté's Arrested Adulthood (2000), Jeffrey Jensen Arnett's Emerging Adulthood (2004) and "The Long and Leisurely Route: Coming of Age in Europe Today" (2007), Harry Blatterer's Coming of Age in Times of Uncertainty (2009), 
Konstam's Emerging and Young Adulthood (2015), and others. Some scholars have presented lists of markers from surveys; others have outlined dominant aspects that in the main characterise adulthood in relation to other ages from a particular academic perspective; and still others have discussed the conditions of adulthood more broadly. The qualities and attributes that are associated with adulthood in the writings of the abovementioned scholars range from having safe sex and getting a driver's licence to autonomy of mind and awareness of the complexities of the world. These have been sorted and categorised, and the ones that I thought related to the same phenomenon have been merged. I also excluded markers that were too specific to be useful. In the end, four groups of markers of adulthood were defined, related to structural, social adaptive, worldview orientating, and personal agentive dimensions of adulthood (see table 2). These rubrics have the advantage that they place the four dominant markers that people often associate with adulthood under one heading each: financial independence from parents, a less self-oriented mind and greater consideration for others, solid personal beliefs and values, and being able to accept responsibility for the consequences of one's actions. ${ }^{?}$

The first category of markers in my model consists of structural social factors that mark an official status of full partnership (group 1 in table 2). These markers entail formal and financial autonomy from parents and the capacity to handle one's own affairs, to form a family, to have a home of one's own, to have a job and meaningful leisure time, stable social networks, and recognition from other adults. Since these factors are fairly easy to observe, and because of the shared structural character of these markers, I also include "recognition of equal adult status from others" in this group.

The second category of markers has to do with underlying social adaptive capacities (group 2 in table 2). The markers in this category are related to communal

9 In some studies, particularities like not committing petty crimes and no drink-driving (Arnett) rank high, but these are the four major factors that repeatedly dominate the various overviews. 
interests as a feature, which first leads to the importance of conformity to social rules and norms. Another aspect involves a cooperative attitude, an ability to adapt to other people and to different social situations. These markers are separated, since it is possible to conform to general norms without being able to adapt to variations. In turn, consideration and care for others are vital parts of this dimension. In connection with stable and close relationships, intimacy and loyalty are also commonly regarded as markers of adulthood.

The last two categories concern the inner aspects of an individual's adulthood. Adulthood is generally equated with having a stable worldview, encompassing a set of values, moral principles, and ideas for guidance, as well as an awareness of the complexities of life (group 3 in table 2). In consequence, an adult is said to have reached a point in life when a sense of stability regarding purpose and meaning in life is established, along with a capacity for reflexivity or self-awareness. The last marker in this category concerns a relatively stable emotional life, and emotional control. This means that emotional intelligence is expected, and not that all expressions of emotion are excluded.

Finally, adulthood relates, as a concept, to a personal agentive category of markers (group 4 in table 2). In part, this refers to an independent and rational way of thinking, making judgements, choices, and decisions. The prototypical adult also acts rationally in accordance with set goals and priorities. Thus, acquired knowledge and experiences are put to use for practical purposes and problem solving in everyday life. The last marker illustrates the adult acceptance of responsibility for the consequences of one's actions.

The four groups of markers thus represent various qualitative aspects, or dimensions, that are commonly used when adulthood is discussed. As stated above, no person can, at all times, live up to the standards that these markers entail in their extremes. Contrariwise, a person who tries to do so will fail to be adult, because these dimensions of adulthood are interdependent to the degree that compromises are necessary, which makes awareness of complexities a key marker. Hence, the markers 
cannot be totally isolated from each other, but demonstrate that categorisations of markers pertaining to human qualities may resist unequivocal demarcations. Thematic categorisations serve a rather more practical purpose: as a guideline for tracing, demonstrating, and understanding the complexity and affordances of surface patterns. Naturally, the set of thematic markers I use is not in any way absolute; it is merely one of many possible models. For instance, adulthood is commonly equated with a reasonably consistent performance of stability, reliability, responsibility, and autonomy, which makes a person fairly predictable for others, and it makes the world reasonably predictable for adult persons themselves. Consequently, those qualities in some way permeate all individual markers of adulthood, as do the relationships between the social and the individual dimensions of life, and between the mental and the practical human capacities. In the first article that is part of this dissertation, the markers of adulthood merely feature as a backdrop; in the second they form a more overt frame of reference, as the thematic implications of adaptations are discussed. In the other three articles, the model for markers of adulthood lends a thematic structure for an analysis of the novels, screenplays, and films in focus. By way of example, those articles demonstrate the application of thematic markers in the analysis of rhetorical lines of argument in fiction, especially with the purpose of understanding what happens to the potential meanings of a story in the novel-film adaptation context. 
Table 2

A model structure for markers of adulthood.

1. Structural dimension

a. Financial autonomy from parents

b. Capacity to run a household and form a family

c. Having a job and meaningful leisure time

d. Stable social networks

e. Recognition of equal adult status by others

2. The social adaptive dimension

a. Conformity to social rules and norms

b. Cooperative attitude and social adaptability

c. Care for others

d. Capacity for intimacy and loyalty

3. Worldview orientating dimension

a. Stable set of values, principles, and beliefs

b. Awareness of the complexities of life

c. Self-awareness

d. Stable emotional life and self-control

4. Personal agentive dimension

a. Independent, goal oriented and rational thinking

b. Responsible judgements, choices and decisions

c. Rational actions in line with thinking.

d. Acceptance of responsibility for consequences 


\section{Chapter 4}

\section{The Five Articles of the Dissertation and Their Implications}

In this chapter, I briefly summarise the five articles, which are attached at full length in appendices 1-5, and discuss their respective contribution to the dissertation. Four of the five articles have been published, or as in one case scheduled for for publication in double-blind peer-reviewed publications. The fifth article was regarded as publishable after revisions and has been submitted again. Although each article presents its own argument, they form a more comprehensive line of reasoning if they are read in the order that they are presented.

\section{Article 1: "Strange Masks of Adapted Identities in Patrick McCabe's Winterwood and The Holy City"}

The first article, "Strange Masks of Identities," is published in Adaptation. The text interrogates the Irish novelist Patrick McCabe's dramatisations of how people, in their attempts to adapt in a changing world, make use of character archetypes in media and fictional stories as ideals for their own adult self-identities. These concerns are especially notable in two of McCabe's later novels, The Holy City and Winterwood, in which the protagonists migrate, mentally or physically, in their attempts to adapt to new environments. Since they do so by constructing their identities as narrative adaptations of already existing media characters, the protagonists have to struggle to harmonise the adaptation of their self-narratives, self-identities, and new life patterns with the developments of their surrounding worlds. Above all, the two novels problematise the conditions for social adaptation, how people make use of stories, protagonists' processes of social adaptation, and their subsequent rearrangement of the themes that dominate their life-stories. Neither The Holy City nor Winterwood has been adapted for the screen, but they thematise the correlation between media and social adaptation. McCabe's novels, I argue, illustrate how the construction of identity is defined by thematic narration, and how a world in flux demands a constant renegotiation of that narrative. For the reader, Winterwood and The Holy City may thus 
come with an invitation to consider the analogy between social and media adaptations, and the themes of mediated narratives. In particular, the characters' strategic choices as fictional beings and as narrators thus illustrate the sophisticated and double-edged potential of media adaptations to function as grounds for reflection as regards social adaptation.

When we read and watch fiction, I argue in this article, we carry with us the intuitive notion that identity and the conditions of belonging are not set in stone. As the world around us continues to change, fictional characters offer us temporary costumes that we may try on in our quest to find a balance in life between identity, authenticity, origin, and stability, on the one hand, and, on the other hand, selfnarration, simulacrum, novelty, and development. Because box office and sales charts, book clubs, the interfaces of Netflix, HBO, and other streaming sites beg of us to choose the fictional friends they promote, we are at risk of being appropriated as extended selves for these characters. Patrick McCabe's novels Winterwood and The Holy City also make their readers aware that our habitual choices of fictional friends might enforce the illusion of a comforting permanence, as if the thematic costumes that they offer us are static, will never change, go out of fashion, or wear out. At the heart of this, McCabe's novels suggest, lies a nostalgic yearning for the simplicity of having our own essential selves, as if these and their thematic constitution had been given to us when we were conceived. Yet, as I indicate with anecdotal evidence, not even authors can fathom the precise identity of their characters, because fictional beings share with real people the circumstance that they are not merely products of authorial, parental ambitions. Insofar as literary and cinematic characters cannot be understood without any inferences from our real social world, they interact with the discursive environments in which their stories are created and distributed and in which they develop. This is even truer when fictional characters are adapted for a new medium, time, or place. Therefore, this is in a sense an evolutionary, formative process of transformation, which does not accommodate any desire for simplicity or stability, not least because it is not a one-directional, linear process. It is instead multi- 
directional, creative, and normative all at once, which is part of the pleasure that the adaptation audiences are invited to indulge in, and so are the philosophical questions about truth and identity, as a consequence of the ontological breaches and epistemological fragmentations that adaptations entail. If we construe our identities, like Patrick McCabe's characters, as text fragments that are unrelated to what resides outside their covers, we may lose our way. If, on the other hand, we include the outside in the text, and the text in the outside, as adaptations encourage us to do, our minds may be open to the re-imagination of ourselves and to new dimensions of social adaptation. Adaptations thus foster the notion of becoming selves through self-narration, instead of being a given identity. Thus, adaptations ask us to be adults in our minds, wary of our social, adaptive, collaborative, empathetic skills, conscious of the complexities of the social world and of ourselves, rational and responsible with a greater context in mind.

However, as McCabe's protagonists exemplify, we are all too often tempted to appropriate characters and pre-configured thematic formations and fit them into our pre-existing patterns of analysis, or to make use of them as stereotypical ideals, both as consumers of fiction and as scholars, I would argue. In such instances, the possibility of learning from them to the full escapes us if we treat them as distant acquaintances rather than as friends, and thus forget to be empathetic and respect them as fictional beings. With an adaptation-minded approach, we may instead acknowledge characters' past and present, the shapes that they have taken in different settings, and that they too have multiple self-identities, while they are still but one person. In doing so we may understand the thematic complexities that they embody as fictional beings. Consequently, we may regard adaptation characters as representative of our own ever-present processes of adaptation through life to the thematic circumstances and issues that we encounter and grapple with. Thus, if we accept the premise that media adaptations and social adaptations rely on the same core phenomena, adaptation characters can serve as metaphorical mirrors for anyone who wants to understand their own circumstances, and whom they can become and 
be in a changing world. In particular, adaptations can be understood as narrative metaphors for the theme of adaptation in life. Perhaps adaptations may also teach us to accept ourselves and others better, if we are mindful of our own self-identities as adapted narrative creations. In the end, what Patrick McCabe's characters ultimately tell us, I argue, is that adulthood is a process of adaptation.

\section{Article 2: "Characters as Fictional Migrants: Atonement, Adaptation and the Screenplay Process"}

The second article in this compilation thesis, "Characters as Fictional Migrants: Atonement, Adaptation and the Screenplay Process," is published in the Journal of Screenwriting. The article focuses on the six distinct complete screenplay versions for the adaptation of Ian McEwan's novel Atonement that Christopher Hampton wrote before the project was completed. Between the third and fourth drafts, there was a change of directors, and in 2007 the film finally reached the theatres. Throughout the adaptation process, Ian McEwan commented on all the screenplay drafts. Together, the annotated drafts illustrate how characters transform in the process of migrating from one media world to another, in the novel-to-film process, most notably with the screenplay at the centre of the transformation, while the characters are affected by various external forces. The article thus proposes a migratory perspective on the adaptation of characters and themes, with the screenplay as a vital instrument to understand the adaptations both as processes and as objects. It also develops the idea suggested earlier that migrating characters, in their capacity as social fictional beings with metaphorical functions, and the thematic issues that they represent, adapt to the new media environments, as well as appropriate them, in order to maintain and develop different parts of their self-identities. In tandem, they are appropriated by new creative forces and by the conventions of the new media environments, which in turn must adapt to the characters. As a result, the representation of the thematic understanding is transformed. 
In this article, I thus turn to two of the protagonists in Atonement, Robbie and Cecilia, to follow them as fictional beings as they migrate from the land of the novel, through seven complete screenplay versions, to the screen. Accordingly, this article is concerned with the actual process of adaptation and with what happens to the representation of a theme, in particular the example theme of adulthood, during that transformation. The study indicates that, as the adaptation developments progress, the characters and the transformation of their qualities as adults go through distinct phases, which can be associated both with migratory and problem solving processes the way these are theoretically described by scholars. In particular, the migratory crises that the characters and their development display through the screenplay drafts correspond to the crisis and trauma reactions that migrants may go through with regard to their sense of self-identity.

What the screenplays illustrate is that the characters' development as fictional beings, as they migrate from one medium to another, and the screenwriter's procedures to adapt and appropriate both characters and media are interconnected. In part, this might be because the characters too adapt and appropriate the medium and its creative forces. One of the consequences is that there is an agentive drift from the screenwriter to the characters at play. On that account, Leitch has suggested that novels, screenplays, and films may metaphorically want to become new and enriched versions of their old selves ("What Movies"), in accordance with MacDonald's notion of the screen idea. This may stimulate a decisive step away from the past versions of the characters. However, like migrants and most developing human beings, they also want to remain the same as they imagine themselves to have been before. As long as the characters and their developments also perform thematic functions, this means that the representation of the thematic understanding is influenced both by a progressive movement and by a conservative ambition to leave it unaffected.

In the adaptation process, the characters initially, like many migrants, are not observant of the complexities that migration involves and they see little need to 
adjust their own character to accommodate others. Thus, after leaving the novel, on their arrival in the screenplay world, the characters hardly adapt at all to the new setting. However, their self-esteem folds quite soon in the adaptation process, as do their beliefs and emotional balance. In the screenplay, they become prone to conflict rather than to cooperation, while they seem to be at odds with the adapting screenwriter as well. At this stage, they have almost lost their self-identities and statuses as adults. After these crises, their goal orientation, rationality, and responsibility are brought to the fore to manage the situation, with the result that they re-establish a subservient, cooperative and adaptable frame of mind, together with a raised awareness of the complexities that the migration process entails. However, they have not yet accomplished a full restoration of their self-esteem and sense of community, problems that must be resolved in the next step of the migratory journey, before they can act as adults again and be acknowledged as such in the new environment. In the course of events, other particular markers of adulthood are tested and falter as well, but the Atonement example indicates that the adult personality that a character represents may not necessarily change significantly from novel to film, while the thematic development is all the more dramatic during the process of developing the screenplay.

Yet, no equilibrium between the markers of adulthood can be guaranteed in the adaptation process, since the transformative migration journey also greatly resembles the uncertain developmental progression of the hero's journey. Regarding adaptations, looking upon characters as migrants thus reveals the mechanics that make so uncertain the particularities of the characters' final manifestation and representation of adulthood, in relation to the adult characters they were when they started their migratory journey. As Campbell notes, a character may stop its progress and change directions at several points in the journey. Still, like real life migrants, after all their self-doubts, most of the characters return to what they might have perceived as a core of their narrative self-identities, once they realise that the ideals of the social world are based on a widespread collective, cultural code, that of being 
an adult human being. When they do not, audiences tend to react, and perhaps the recurring outcry about the infidelities of adaptations is related to a desire to view identity and thematic truths as static, for the sake of convenience.

Returning to the evolutionary, as well as the phenomenological, balance between what has been and what is to come, the characters represent an element that has never before been seen in their new environments once they have migrated. Thus, they contribute to an expansion of what it is possible to express in that world, in accordance with Jacques Rancière's definition of the distribution of the sensible (12). Likewise, the thematic representation is expanded with each new version of a story.

In effect, as I have previously touched upon, film conventions and characters have both been affected by the adaptation process. This bi-directional acculturalisation, in the sense that both the migrating element and the host setting adapt and change, also involves appropriation. To some extent the characters must appropriate the new narrative settings and their conventions, which must accommodate and adapt for the sake of the characters' survival. Meanwhile, the screenplay and film environments must partly appropriate the characters for their narrative purposes. The effect is ultimately a three-way exchange and negotiation between the characters, the creative forces, and the narrative conventions, which involves mutual adaptations, appropriations, and accommodations. Hence, if we, as scholars, employ our imagination to consider the characters empathetically as fictional migrants, the migrant perspective on adaptations may also expand our comprehension of the political and social world that we inhabit.

\section{Article 3: "Adaptations of Adulthood: Towards a Model for Thematic}

\section{Rhetoric in Adaptation Studies"}

The third article, "Adaptations of Adulthood: Towards a Model for Thematic Rhetoric in Adaptation Studies," appears in New Approaches to Contemporary Adaptation (2020), edited by Betty Kaklamanidou for Wayne State University Press. It elaborates 
and tests the model described in chapter 3 for the study of thematic, rhetorical lines of argument in narratives, based on the hero's journey, and on ten successful novelfilm adaptations, in relation to the representation of the example theme adulthood through the protagonists' thematic functions. ${ }^{10}$ Schematically, the article identifies patterns in the representation of the thematic markers of adulthood at different stages of the narratives. The article subsequently proposes a foundation for a theory of thematic lines of argument, with a theoretical stance derived from Cardwell's discussion in Adaptation Revisited about adaptations as story universes revolving around what she defines as a mythic ur-text: a mental and variable construct that serves as a node for versions of a story. In this case, the ur-text is comprised of what can be likened to an archetypal human understanding of the example theme, adulthood.

Most inspiringly, Cardwell's ur-text hypothesis replaces the hierarchical and linear novel-to-film model with a relationship between different versions based on equality, regardless of media form, time of production, and order of audience experience. Furthermore, if the notion of linear media processes of adaptation is abandoned, the idea of the ur-text has the potential to satisfy our natural desire for a connection and harmony with a greater original existential source, whether it be nature, evolution, God, or civilisation, without adding to any reductive essentialist strand of thought. Thus, the quest for an original text, the mythic ur-text, eventually unites evolutionary and post-modern ways of thinking, since the resolution is that the ultimate representation of the abstract ur-text is the thematic, rhetorical, meaning making constitution of stories.

The ten novels and films in this article are regarded as two representative corpora and not as ten individual adaptation pairs. The purpose is partly to address the issue of media-specific conventions in relation to the thematic content, the representation of thematic markers of adulthood, and the rhetorical plot structure.

\footnotetext{
${ }^{10}$ The titles in the sample are as follows: Atonement, Fifty Shades of Grey, Gone Girl, Me Before You, Room, Shutter Island, The Da Vinci Code, The Martian, The Road, and Up in the Air.
} 
Although the sample is too small to produce any statistically valid evidence of patterns and variations, for a qualitative assessment the ten titles tentatively form an illustrative example. When the main characters' actions, reactions, expressions of thoughts, and utterances are coded and analysed, the representation of the various markers of adulthood does not differ significantly between the novels and films. Although there are noteworthy differences between a specific novel and its film sibling, and between two novels, or two films, the results regarding the corpora suggest that the distributions of adulthood markers are largely similar in films and novels, and that they are equally complex as far as the construction of the thematic line of arguments is concerned. If nothing else, this indicates that the models and thematic representations are media-neutral, even in light of the narrative conventions of novels, screenplays, and films.

However, there are certain non-conformities. Compared with the novels, the films emphasise social markers, as well as the importance of home and family. This might be a consequence of the cinematic convention of giving precedence to the visualisations of interactions, whereas novels may, to a greater extent, leave those aspects as gaps that readers fill in through their mental visualisations. Novels, screenplays, and films equally depend on readers' and spectator's abilities to fill the gaps, by considering the way that expressive means are used to cue thematic meaning and bring forth the intricacies and richness in thematic content that each medium permits. Also, the novels accentuate, more than the films, overt instances of the characters' awareness of complexities, as well as, for example, the display of values and beliefs. Nevertheless, the films often compensate for this with expressive impact when these aspects of adulthood are in focus.

Since the ten novel-film pairs include a wide variety of titles, ranging from Atonement and The Road to Fifty Shades of Grey and The Da Vinci Code, the sample represents a spectrum of successful adaptation pairs. Most of them were adapted for the screen with the ambition of being faithful to the readers' expectations, but some, like $U_{p}$ in the Air, were adapted with less adherence to the novels' structures. The 
variety of the adaptations in the sample is both open to broad generalisations and limits the reach of the conclusions. Returning to the various taxonomies that I refer to in chapter 3, the results do not, for example, indicate how transposition adaptations and revisionists' adaptations differ as regards the management of thematic issues or the representation of adulthood in particular.

Still, what surprises me - partly due to my own prejudice - is the structured and intricate constitution of the rhetorical line of argument about what it is to be adult in examples of blockbuster novels, screenplays, and films such as The Da Vinci Code and Fifty Shades of Grey. Again, the sample is too limited to generalise, but the findings imply that genre, medium, and their relationship to market values matter less when it comes to the representation of adulthood in fiction than do other factors, like creative ambitions and ideologies.

\section{Article 4: "Okay: The Road and the Good Guys' Adulthood Code"}

The fourth article, "Okay: The Road and The Good Guys' Adulthood Code," to be published in The Cormac McCarthy Journal, employs the same set of markers and the same model for the thematic, rhetorical line of argument as the third article, but for a single title: The Road. Cormac McCarthy's overtly eco-critical novel The Road is highly relevant when the theme of adulthood is in focus. It follows a father and his eleven-year-old son on their journey on foot through a post-apocalyptic wasteland. As they travel, the dying man has to learn anew what it is to be adult, in order to be able to pass that knowledge on to his son. The screen adaptation transforms the unconventional form and poetry of the novel to a film that matches the standards of post-apocalyptic road movies, while the screenplay conforms to the poetic condensation that characterises the screenplay form. In all three versions, the word okay is used in the dialogue in a dramaturgical rhythm to emphasise thematically relevant instances. As mentioned above, the hero's journey is once again applied as a structure to compare how the development of a thematic concept, adulthood, is plotted in novel, screenplay, and film, with a particular focus on the first two. Thus, 
the article contributes to a further understanding of the thematic, rhetorical line of argument in narratives, and, to some extent, it explores the transformation of the specific poetic form of the novel to the screenplay adaptation, which must inherently also adapt to the screen idea.

The Road is less than half the length of Atonement, as far as the novels are concerned. McCarthy's novel is regarded as being quintessentially American, and is written in a language that may evoke the experience of reading a long poem, whereas Atonement is written with the conventions of classic British novels in mind. However, the adapting screenwriters, Joe Penhall and Christopher Hampton, are both British, and The Road's director, John Hillcoat, is Australian, while Atonement's director, Joe Wright, is British. As a film, The Road has obvious links to dystopic films and road movies, while Atonement is often regarded as a heritage film. In a sense, both films are thus produced in the American Indiewood tradition, as it is described by Geoff King. In that context, The Road can also be described as an eco-critical survival drama, and Atonement may be regarded as an intellectual love story. However, both of them thematise adulthood with children's development as backdrops, and they contextualise adulthood by placing the protagonists in settings that are conditioned by the aftermath of what humankind's destructive powers have resulted in. In addition, as in the case of Atonement, the novel, screenplay, and film The Road all disclose who the narrator is at the end of the story, which initiates a return to the narratives' beginnings and a reconceptualisation of the lessons learned.

The focus in this article on a particular rhetorical cue, the word okay, and the attention it draws to specific markers of adulthood, reveals how the adaptation of certain expressive means affects rhetorical structures. Above all, the novel foregrounds a dialogic approach, whereas the screenplay and film put a dialectic rhetoric to work. This might be a result of the cinematic tradition of presenting clear antagonist-protagonist relationships and the convention of engaging visually tangible contrasts as part of the screen idea. If that is the case, such strategies are nevertheless stimulated by the novel, which initiates the contrast between the good and bad guys, 
and the materialisations of surfaces, physical sensations, and movements by means of Cormac McCarthy's prose. The effect is that the novel, screenplay, and film versions produce complementary insights regarding what it is to be an adult, and they do so as a result of creative choices. As, for instance, Terrence Malick has demonstrated with films like The Tree of Life, which is partly produced by the same team that produced The Road and The Thin Red Line, the phenomenological lyricism of Cormac McCarthy's novel is not restricted to the form of the novel. Nor are Dan Brown's novels, with their rapid editing pace as one scene and chapter is replaced with another, less novelistic just because the author makes use of narrative strategies that are commonly associated with cinema. As The Road exemplifies, the strategic choices regarding discourse and poetics may, however, affect the thematic representation, above all when readers and spectators are expected to consider the narrative discourses to piece together the particularities of the narratives and thus shape notions of greater wholes. To continue the comparison of Atonement and The Road, the rhetorically and thematically important endings are the obvious results of creative choices. For example, in the case of The Road, the internal-spiritual dimension of the novel's hopeful end is replaced, in the screenplay and above all in the film, with an externalised, social aspect, with the sensations of hope and belonging still well-defined.

However, The Road also demonstrates that the media-specific conventions allow the translation of a thematic rhetoric from the novel's means of expression, to the poetics and conventions of the screenplay, and thence to the audio-visual modes of the film. That does not mean that this has always been accomplished to the extent that the screenwriter or director desired. Again, what may affect the thematic result more than the media conventions are the people, the creative forces, who are involved in the projects: writers, advisors, the readers they have in mind, actors, producers, cinematographers, directors, and others. 


\section{Article 5: "Adaptations and Post-apocalyptic Atonement"}

The final article, "Adaptations and Post-apocalyptic Atonement," has been submitted for publication to Literature Film Quarterly. It returns to the question of the reception of adaptations and explores how the thematic representations of adulthood in the novel, screenplay, and film Atonement may be open to what can be referred to as a post-apocalyptic imagination or reading, which disrupts linear causality and places all events, versions of a story reflections, and meanings side-by-side, mobile and in stable harmony at the same time, so that they may disclose a holistic thematic awareness. If the previous articles in the dissertation, especially the second, indicate that adaptations are bi-directional, in the sense that media and social adaptations, the various versions of an adapted story, are interdependent, this final article thus expands on the non-linear constitution of adaptations and suggests a postapocalyptic perspective to define the nature of adaptations.

On the surface, the story of Atonement does not answer to the generic description of the post-apocalyptic genre, as it is popularly defined: speculative narratives about survival and lost community with events set in a devastated landscape in a possible future. The qualifiers are all present in the narrative, but it is the Second World War that serves as a backdrop, instead of an unidentified catastrophic event. Accordingly, post-apocalyptic fiction is, in this article, defined in a broader sense, with a reference to a biblical notion of the post-apocalypse. It refers to the notion of a new heaven and a new earth after the end, as well as to a state of being beyond what is previously known, an existence in which perception and cognition are no longer bound by time, causality, space, or materiality. In this context, a post-apocalyptic vision thus denotes the ethical and thematic order that is revealed after the apocalypse, or the end of a story: namely the eternal beginning of relational, narrative meaning. A corresponding post-apocalyptic view on normalcy embraces the possibility of merging the past and the opportunities of the future into one narrative, to consider all our tentative self-narratives in tandem. It also implies that happenstance developments and constancy may be parts of the same whole. Thus, 
the post-apocalyptic vision dissolves the linearity of time and place, and it unites all elements, which may once have appeared to be inseparable, into a larger thematic whole, so that gaps are filled and greater understandings can be revealed.

Post-apocalyptic imagination can thus be compared with Jürgen Habermas' reflection on the relationship between modernism and post-modernism, namely that the cultural project of the past two hundred years has explored the possibility of the human mind-set fusing the rational with the aesthetic, instead of separating them, and of establishing a discourse to consider the concept of sense and sensibility rather than sense or sensibility. As Atonement suggests, this involves the unity of the emotional or aesthetic identity with the rational or pragmatic one, as well as of the individual and communal aspects of the self, which makes it possible for us to sense that we belong. Again, what adaptations foreground, when the complex structure of Atonement is considered, is the human desire for a state of restored normalcy and the freedom of agency and awareness that it might entail.

For audiences to have an adaptational mind-set, I maintain, is thus equivalent to having a post-apocalyptic approach to thematic reading, watching, and understanding. In both cases, allowances are made for a consistent oscillation between alternative versions, which may at best produce the experience of entropic balance as a state of normalcy. The adaptational mind may thus construe the experience of alternative potential developments and self-identities as a form of happy ending.

In the novel Atonement, as well as in the published screenplay and the film, the narrative construction represents and encourages such an adaptational mind-set, because that is what it is concerned with. Atonement thus also stimulates a holistic perspective, not least due to its structure and discourse, based on the varied points of view and the multi-layered intertextual mind games that present themselves in all the versions. On a surface level, the novel's many explicit references to literature and narration, such as to the works of Virginia Woolf, Henry Fielding, and Samuel Richardson, are, in the screenplay and film, accompanied and often replaced with 
references to the films directed by Marcel Carné, David Lean, and Anthony Asquith, among others. For the knowing or curious audience, these references prompt further investigations about and oscillations between the readers' and spectators' own selfidentities and the representations of the characters.

Like many post-modern narratives, Atonement employs the narrator to thwart the notion of borders between narratological ontological borders. In spite of its adherence to conventional plot structures, Atonement thus forces the reader and viewer to abandon the linearity of the narrative and rely on circular references to fully comprehend the complexity of the happy ending that a truly post-apocalyptic story is all about. Hence, Atonements narrative characteristics also exemplify, as Bordwell argues, that beneath the most complex narratives lies a rhetorical hyper-structure (The Way) which, I argue, is concerned with the theme of the narratives. In this context, the apocalyptic understanding of adaptation particularly motivates the use of a Campbellian perspective on the narratives, since the essence of the monomyth, the hero's journey, is the ambition to accomplish an experience of unity in a world which often seems to be fragmented into opposites. When the distributions of thematic markers are studied through the filter of the rhetorical plot structure, it is clear that novels, screenplays, and films may part ways, no matter how faithful writers, directors, and producers intend to be. In particular, because the beginnings produce the premises for the thematic arguments and the endings communicate the conclusions, together they control the perception of the alternative versions of the thematic arguments in the narratives. In Atonement, this comes with a twist, since the post-apocalyptic and adaptational mind is asked at the end to return to the beginning to construct ever new wholes.

Accordingly, Atonement dramatises the possibility of choosing between alternative versions of a narrative as a foundation for a sense of self and belonging. Through the course of the narration, layers upon layers of forking paths for alternative stories are produced, with overt alternative endings around which the narrative may hinge. Thus, with the screenplay and film adaptations, which adhere 
to this structure, the message and the grounds presented for thematic reflection beg of the adaptation audience not to indicate a preference, but to consider the relationship between the alternative compositions of the narratives and their representations of adulthood as dialogic, with awareness of complexities as a central node.

Thus, whether we read the novel or screenplay, or watch the film, each of the narratives proposes alternative elixirs of happiness, and different blends of markers of adulthood, but ultimately Atonement calls for more than a consideration of the conclusion. It motivates us to regard the line or lines of argument in their entirety as part of the formula. In many works of fiction, the presence of a thematic argument and its structure may be completely obscured, while in other cases, the thematic representation may be over-simplified. Yet, as long as there is an adaptation, the complementary versions summon us to compare and evaluate in order to develop integrated and multifaceted insights. Thus, even the simplest of narratives may instigate complex mind games that are concerned with existential issues, like the constitution of and personal relationship to adulthood, for audiences to consider and engage in. 


\section{Chapter 5}

\section{Concluding Remarks: The Adapting Story Resumes}

I started out with the simple question of whether or not it matters which film adaptation, or version of a story, people take to their hearts and allow to affect their minds when they wonder who they are and who they can become in relation to the social world that they inhabit. Adapting a novel to a screenplay and subsequently to a feature film takes time. Thus, historical events that occur between the productions of two versions of a narrative may affect the discursive and social frames that circumscribe thematic reflections. This is significant since screenwriters, directors, producers, and others who are involved in the adaptation process are sensitive to the values of their cultural environments. However, media productions also affect the political and social world in which they are produced. Accordingly, I have contended that the thematic adaptation is a result of a multi-dimensional, bi-directional exchange, which also concerns the mutual, normative exchange between the social and the fictional worlds. The nuances that dominate conversations about a specific theme, such as adulthood, have an impact on thematic adaptations, while any adaptation likewise affects the dominant notion of a theme among the members of the audiences.

In this dissertation, I have therefore considered the connection between media and social adaptations, and I have primarily asked what happens to the representation of themes in the process of adaptation when novels are adapted for the screen. The simple answer is that each version of a story produces distinct lines of reasoning about the thematic constitution and significance of a theme, since every adaptation is affected by its discursive environment. Therefore, there are individual nuances regarding the weight, balance, and functions of relevant thematic markers, which may serve to describe the facets of the representation of a theme in every version of an adapted story. Consequently, each version generates its own conditions for audiences' reflections and meaning making processes. Nonetheless, films, screenplays, and novels all have the potential to display a rich representation and 
complex lines of thematic reasoning. That said, fictional narratives do not necessarily affect readers' and spectators' self-awareness, personality, and values. Yet for readers and spectators who actively reflect upon their own relationship to a theme, especially a theme like adulthood, adaptations offer particularly multi-layered grounds for reflection, since they offer more than one narrative version of a subject. In effect, they also accentuate that thematic understandings are inherently provisional, always in transition, subject to discursive exchanges.

What changes most when the representation of a theme is adapted from novel to film is the mode of representation, since the verbal novel must first be distilled to the poetics of the screenplay, before the narrative is subsequently unfolded and given a new form of density as a multimodal film. However, novels, screenplays, and films generally share a repertoire of expressive means. I have previously stated the obvious fact that as long as characters speak in films, it is not exclusively a literary prerogative to express thoughts and feelings verbally. Voice-overs and overt narrators may also fill the role of a literary, verbal narrator, as is the case in both The Road and Atonement, for instance. Nevertheless, as Elliott, among others, has argued, literary representation is fundamentally based on verbal signs, from which mental concepts and images are produced, whereas cognitive meaning making for the film audience, apart from verbal expressions, conventionally begins with audio-visual perceptions from which concepts may take the shape of verbalised thoughts (202). In both cases, the audience experiences a cognitive exchange between the perceptual and the verbal, and the enhanced stimuli produce emotional and bodily responses, which in turn may or may not provoke conscious thoughts.

Yet, it is obvious that the appreciation of representations of themes through the attributes and functions of characters in fictional narratives depends on their audiences' capacities and habits of filling gaps, reading minds, and construing meaning from fragments. Most people exercise this competence with varying degrees of excellence on an everyday basis in real life, when they read situations, places, and to some extent other people's minds as a natural part of any social exchange, and, as 
Zunshine submits, this is how we understand literary fictional beings as well. The characters' values, beliefs, thoughts, and emotions may be expressed by the narrator as inner dialogue, by other characters, or may be reported, more or less reliably, by the characters, but the understanding of a character's thematic contribution is mainly a matter of the puzzle of fragments that a theory of mind explains. The multimodal richness of the film medium, with its distinct framing, cinematography, mise-enscene, editing, sound, and moving visual representations of the physical world, cues similar mental activities and enables the spectator to decipher a character's feelings, conceptual convictions, and states of mind. The intricate interplay of meanings that the semiotic resources of film may thus produce are certainly equivalent to the delicate richness of linguistic expressions that are used in novels to represent adulthood, or any other theme.

It is worth mentioning that the digital era has made it easier to overcome many problems in the production of an adaptation. It was once true that literary narratives and characters could change locations more easily than they could do in films, just as novels could fill a space with any number of people, while films used to be bound by physical and financial restraints, as well as by actors' physical appearances. The digital possibilities of editing and framing images, sound, and even actors have given creative film production teams the potential to match the freedom of the creative writer. All things considered, although the representation of adulthood is strongly affected by the modes of representation, the possible alterations in thematic content are not due to specific conventions of narrative structures or to any media specific limitations, but are the results of the discourses that surround the production of the narratives and the creative forces that produce them.

As proposed earlier, the creative teams both adapt to and appropriate the thematic potentials and fictional ambitions of the characters, who in turn adapt to and appropriate the thematic potential and views of adulthood that a certain author, screenwriter, or director may have. Because they must aid the thematic concepts and the characters through multiple migratory experiences to new places in time, space, 
and media forms, the formation of a representation of adulthood during the course of the adaptation process becomes a matter of complex problem solving for the creative teams. What happens to the characters' thematic dimensions and attributes, and in the end to the representation of adulthood, in the process thus becomes a matter of negotiation, compromise, and prioritisation, which is also valid for the representation in fiction of the composition of adulthood as an ideal and as a state of normalcy.

There is, I would argue, perhaps an even greater conflict between the idealising notions of a theme and the ways thematic understandings are revealed through practice in life, than between a theme as it is represented in films, screenplays and novels. This may be particularly true when a theme is so closely linked to what may be perceived as a person's self-identity as the theme of adulthood is, according to Blatterer (Coming 27). Fictional narratives thus seem to illustrate this divide and problematise the concept of adulthood and the contrast between practical and idealising constructs of thematic understandings. In the case of adulthood, due to increasing social complexities, with demands and pressures on and from networks, families, responsibilities, careers, social security, norms, and conventions, adulthood cannot be considered to be a stable phase of life. Not even the stability of networks, workplaces, financial independence, and family relations are statistical normalities that can be used to describe adulthood in the western world, as is widely illustrated in contemporary films, screenplays, and novels. Nevertheless, those qualities still constitute the structural foundations for the normative definition of adulthood in contemporary narrative fiction, based on a visionary ideal, which might serve to evoke the hope of future stability and a somewhat happy state of adult normalcy, or to interrogate and problematise the constitution of that stability and of normalcy.

The romantic vision, which people and fictional characters may sometimes share, of adulthood as an unproblematic life stage, when all opportunities may be realised, is often contradicted by the realities that adult life has to offer, as is also the case regarding most other complex themes. The result is often a recurring cognitive 
dissonance. As an alternative, the migratory aspects of adaptations correspond with and thus metaphorically foreground the adult experience of relentless adaptation and development, and in general the adaptive constitution of thematic concepts. Hence, adaptations advance the opportunity to regard adulthood as a less fixed state of being, so that the ideal of what it is to be an adult and what it means to actually go through life as an adult may be harmonised to a higher degree. When versions of a story are compared and regarded as equally valid narrative accounts of adulthood, the phenomenon of alternative representations of the thematic notion thus illuminates that representations of complex themes cannot be generated by the use of a simple formula or a single narrative. Adaptations thereby emphasise the contemporary convention in narrative fiction of contradicting the notion of adulthood as a stable phase of life, free from complications and conflicts. All the everyday impediments and instabilities of adult life seem to be equally accentuated in novels, screenplays, and films, although with varying nuances. Hence, what adaptations do teach us is that our longing for normalcy in adult life propels us forward, but that new intricacies always emerge. Adaptations may thus help us to accept adulthood as a state of certain flux and natural imperfection. The example theme, adulthood, may thus be interpreted as a metaphor for all thematic understandings and knowledge, unstable, adaptive, and both subject to and subjects in a process of development.

As regards the rhetorical presentation of adulthood as thematic content, I have emphasised the importance of the thematic, rhetorical line of argument in narratives, but also to some extent the impact that media specific modes of representation may have on the thematic rhetoric. I have argued that we mainly follow the characters' progression through the plot of the narratives, when we read or watch fiction, and we adjust our empathetic and sympathetic positions strategically in order to be able to identify with the characters and to simulate their experiences mentally, to the various degrees that we find suitable for each encounter. Therefore, inspired by Phelan's theorisation of the relationship between plots, characters, and themes, I 
have translated the hero's journey into a rhetorical line of argument that can be used to track the thematic functions of a character through a narrative.

The study of plot structures may be tiresome and may seem repetitive, but if they are regarded as thematic, rhetorical structures, such studies are productive and open up exciting comparisons between the lines of arguments in different versions of narratives. Further opportunities are revealed if the inclusion, rather than the exclusion, of the screenplay becomes a standard in adaptation studies, which enables the study of both the individual objects and the process of adaptation as a whole. In this context, my findings also address the biased opinion that mainstream films are governed by plot structures, while European so-called art films and novels are not, a view which is still widespread, both in academia and among everyday consumers of fiction. Admittedly, I, too, entered film studies many years ago with the prejudice that film adaptations by necessity simplified and reduced the intricacies of literary characters and the thematic explorations of novels. Yet, as I hope to have shown, within the scope of this dissertation there has been little to suggest that the conventions of literary or cinematic production endorse and affect the thematic message about adulthood in a specific direction. The consideration of a thematic plot structure in the analysis suggests, however, that, regardless of genre and media form, the narratives depend on a thematic line of reasoning, equivalent to the hero's journey. Thus, the deep structure of the rhetorical argument remains when a novel is successfully adapted to a screenplay, and this in turn is adapted into a film. However provocative it may still seem to some, in this respect there is little difference between, for example, complex idea based novels and Hollywood blockbusters, and so called art films. ${ }^{11}$ Consequently, the representation of adulthood is conventionally

\footnotetext{
${ }^{11}$ For example, during the course of this project I supervised a student who, in his BA thesis, took this approach in an analysis of Ruben Östlund's Involuntary and revealed how the film, in spite of its short cut format, conformed to the thematic line of argument that I have promoted in this dissertation (Gustin).
} 
supported, and to some extent controlled by the constancy of structure of the rhetorical line of argument when stories are adapted, which explains why thematic representations can be seen as easily "adaptable across media and even genres or framing contexts," as Hutcheon maintains (10).

However, as indicated, the ideological preferences of the creative forces and the concurrent socio-cultural trends may make the different versions prone to dissimilar emphases, balances, and causalities with regard to the lines of reasoning about adulthood that they pursue. Thus, within the framework of that structure, the orchestration of characters and their thematic qualities may result in variations in the dialogical and dialectical structures of meaning production, as well as in the thematic argument and whatever thematic aspects are selected for emphasis. The framing of the line of argument might also be altered. It can be used to produce a linear argument with a clear conclusion, which may result in a distinct definition of adulthood. Likewise, the choice of narrative frame may also prompt a postapocalyptic, phenomenological, and hermeneutic argument, in which a fixed state of adulthood is questioned, and the rich and dynamic constitution of the concept is foregrounded. In the latter case, the end of the narrative provokes a retrospective and reflexive re-evaluation of the various elements of the argument, in a circular quest for holistic meaning. The representation of adulthood, and other themes that encompass similar complexities, may better correspond to such a holistic perspective and rhetoric, I would argue, since narrative structures that activate the audiences' minds in the meaning making process allow the readers and spectators the agency of competent and responsible adults. In addition, with Sarah Cardwell's ur-text notion as a framework for the analysis of adaptations, the narratives are regarded as equally valuable, as parallel versions, for the reflexive work that the members of the audience are invited to engage in, in order to consider patterns, similarities, and differences in the representations of adulthood as part of their personal development.

In that context, as I have argued above, adaptations are conditioned by compromises, negotiations, and priorities. These dimensions draw attention to the 
idea that adaptations may serve as media metaphors for other thematic markers than merely for the ability to adapt. For example, two markers of adulthood can be said to function as nodes for the others: a person's self-awareness and their awareness of complexities. Metaphorically, these two qualities are inherently demonstrated by all adaptations, since every adaptation displays a degree of consciousness of the narrative's constitution and of the circumstances of the production process, that is to say the narrative's past, present, and possible future. Adaptations may also give rise to questions about responsibilities, rational aims, values, care, cooperation, and so forth. Thus, as long as adaptations are perceived as a set of interrelated versions of a story, its characters, and themes, they communicate that there is no single thematic, narrative truth, and thus no single adult self-identity or personality, since they are all defined by global as well as individual narrations. Hence, adaptations illustrate a holistic and purposeful way of thinking and making meaning, which serves to do justice to the constitution of a complex theme like adulthood. This envisioned adaptive mind-set offers a manner of seeking order by means of organising and maintaining a structure vis-à-vis the narratives of life and thematic insights, while allowances are made for non-linear, bi-directional, and multivariable interconnections and re-negotiations.

As scholars like Raw, Elliott, and Leitch have suggested before me, adaptation studies can be advanced if approaches from the social sciences are applied when the processes that affect media adaptions are studied. In this dissertation, I have mainly referred to social adaption, developmental theories, and migration studies with the evolutionary doctrine in mind, and have argued that media adaptations should be regarded as a reflection of the human condition, with all the thematic concerns that it involves. Therefore, our knowledge from the fields that study human behaviours, cultures, and minds, as well as the methodologies and models that those insights have resulted in, should be put to productive use in media adaptation studies. If the premise is that we, in our capacity as readers and spectators, engage in fiction in order to find grounds for reflection about the conditions that we live in and to come to 
terms with who we can be as adults in that world, Patrick Cattrysse's call for further descriptive adaptation studies is highly relevant. I believe that, in this post-theory era, the most productive stance is to adopt a certain infidelity to theories, with the purpose of inviting productive combinations and cross-fertilisations of systems of ideas and knowledge from fields that may, on the surface, or by their defending champions, be regarded as incompatible. We may then reveal patterns and insights that have hitherto been veiled. Even more stimulating is the prospect of learning more about the human condition through the study of media adaptation studies, when the play of bi-directional reflections between the fields of study is initiated.

As these concluding remarks have indicated, I have, in the pursuit of a fuller answer to what happens to the representation of themes, and adulthood in particular, when contemporary novels are adapted for the screen, addressed questions like why we care about fiction and fictional characters, how we read and watch to satisfy that interest, and how the sprawling concern for adaptations in our culture can be related to those inquiries. No theory of adaptation can yet summarise the current state of affairs in a neat quantitative formula, like $\mathrm{e}=\mathrm{mc}^{2}$, and thus offer assertive conclusions about the details even of any randomly selected formulaic adaptation. The reason is that the relationships between adaptation narratives by necessity depend on interrelationships between the achievements of the creative forces that are engaged in them, on the audiences' cognitive response activities, and on the forever-changing cultural landscape that affects both the productions and their reception. Media adaptation scholars may still define parameters and contexts for specific problems in order to address, discover, and theorise about the ruling principles behind adaptations, and, like other researchers, we philosophise about the constitution of the universe that we are concerned with, the ever expanding world of adaptations. In doing so, we affect its development, as we suggest methodological or theoretical pathways, which may inspire new discoveries. With this dissertation, I have explored patterns and examples in search of trails that may contribute to further progress in a small way. Inasmuch as any multi-disciplinary project inherently reaches across the 
borders that demarcate specific academic fields, adaptation studies has an extraordinary tendency to reveal its rhizomatic nature. Hence, it has been impossible to cover in depth all the aspects that have called for attention during the course of this project. There are, in particular, some aspects of concern for thematic representation in narratives that have not been thoroughly addressed in this dissertation. Like most adaptation scholars, I have touched upon the connection between expressive means and thematic content. However, the purpose has not been to produce an extensive chart of how certain verbal and grammatical forms conventionally prompt specific audio-visual strategies when themes are subjected to creative adaptations. Over the years, narrative conventions from different media and genres have been affected by cross-contamination, so that the designs of novels, screenplays, and films nowadays allow for productive narrative variations. This, however, does not invalidate, but rather renews the curiosity about what insights such a study could reveal.

Moreover, I have not concerned myself much with genre theory, but there are still reasons to believe that different genres may propose alternative views on themes in general and on adulthood in particular, based on the discursive and ideological premises that are attached to different genre conventions. The romance novel, romantic comedies, detective novels, film noir, science fiction, and horror stories all originate from particular ideological foundations, which may affect their views on themes that are interlaced with the conventions of these genres, even in the era of mixed genres. Indeed, many of the fiction titles that this dissertation has been concerned with are, genre-wise, best categorised as hybrids, but they have not been studied as representatives of that development. Likewise, in spite of the communicative approach that I have had in mind, the voices of the novelists, screenwriters, directors, and producers behind the adaptations have not been engaged in this dissertation. Nor have I explored the impact that various strategies and levels of author-narrator communication may have, in practice, on adaptations or on thematic representations. From a communicative aspect, studying audiences' 
responses and idea formations in relation to their reading and watching also remains to be done.

Since my approach has been to regard fictional narratives as structured or plotted lines of arguments, an initial evaluation of various plot models was performed in the pre-study phase of the project. I have not given a full account of that analysis, and have focused entirely on the structure of the hero's journey as presented by Campbell. Nonetheless, I am convinced that most plot models, from Freud's masterplot, as it is described by Brooks, to Truby's twenty-two step plot, can effectively be transformed into rhetorical thematic structures that may highlight other aspects than the hero's journey does. To my knowledge, there is no study that compares the thematic implications of the many plot models in the creative writing manuals on the market.

With these limitations in mind, I find that the models that I have proposed for the analysis of the progression of thematic arguments in narratives can be applied for various research and teaching purposes. The thematic interpretation of the hero's journey works as a complement to the plot models that describe the progression of characters and events in narrative fiction. This media neutral model may thus serve as an analytical tool to teach students that films, screenplays, and novels are equally complex; they are only surrounded by dissimilar conventions with regard to the use of the available semiotic resources. Furthermore, this model may facilitate the need for aspiring authors, screenwriters, and adapters to keep track of the thematic developments in their narratives. In adaptation productions, one of the most intricate problems is that of establishing a communication between authors, screenwriters, and producers about the meaning of the story they are telling. Simple statements of premises and conventional plot descriptions do not suffice to communicate the thematic ambitions that various stakeholders attach to a production. The application of the structure of the thematic line of reasoning that I have proposed may add a helpful dimension to such negotiations, and may help to control the desired thematic content in the production of individual works as well. 
For scholars, students, and creative writers alike, the bi-directional model of adaptation that I have submitted may help us to understand the creative processes and the thematic development of adaptations. An increased awareness that characters and media conventions, and thus creative teams, adapt to and appropriate each other's contributions to the narrative developments may assist in the creative negotiations and prioritisations that all narrative craftsmanship entails. Students and scholars may, if such aspects are taken into consideration, develop a greater sensibility as to why adaptations develop the way they do.

There is much to be gained by turning the analytical pyramid around, as Eder suggests, and by first considering fictional characters as actual, albeit fictional, beings to establish a solid comprehension of their constitutions as such, before asking what their represented ways of being are symptomatic of. In reading and watching adaptations, particularly when the representation of a theme like adulthood is in focus, an adult approach to the enterprise is thus appropriate, calling for an awareness of complexities, responsibility, and independence in any judgements, and an empathetic attitude towards characters and the creative professionals who have been involved in crafting the stories. All too often, both as scholars and as private consumers of fiction, we appropriate characters and fit them into our pre-existing patterns of analysis. The consequence of treating them as distant acquaintances, rather than as friends, and of forgetting to be empathetic and to respect them as fictional beings, is that we may lose the opportunity to learn from them to the full. If narrative fictions in specific adaptations are regarded as grounds for reflection about who we may be and who we may become, we should also acknowledge the characters' past and present states of being, the roles they have taken in different settings, and acknowledge that they have multiple identities, while they are still only one, simultaneously. We should thus keep in mind that most characters have migrated many times between perceptibly complete worlds: a novel, five or six complete screenplay versions, a film, and perhaps even further to a game or a graphic novel, and yet they still keep all those past versions of themselves within, as 
remembered beings who may come to life again. Each time they migrate, they have to adapt their self-identities and also appropriate the worlds they enter, while each world in turn appropriates them and must adapt to their presence to some extent. Hence, the meaning of all of the elements involved expands. If we thus regard these characters empathetically, as migrants with a past, we may better comprehend why they have come to migrate, as well as the personal processes of adaptation that they have gone through in order to become relevant again in the new setting. Perhaps we may even ask not just what they have left behind and how they relate to their former selves, but who they want to be, in their new lives, if all the necessary resources and opportunities are given to them. Then we might recognise what they still carry inside, but have not yet the capacity to disclose. Maybe we could even appreciate their potential contributions to their new habitats. With this approach to characters as fictional beings, adaptation studies and adaptations can rise to their potential as metaphorical mirrors for anyone who wants to understand their own frustration, and who they can become and be in a changing world. Thus, media adaptations may also help us to enhance our understanding of ourselves and others further, and guide some of us to grow into the adults we want to be. 


\section{Works Cited}

Abrahamson, Lenny, director, and Emma Donoghue, screenwriter. Room. Element Pictures, 2015.

Aiken, Lewis. Human development in adulthood. Plenum, 1998.

Altman, Rick. A Theory of Narrative. Columbia UP, 2008.

Andrew, Dudley. Concepts in Film Theory. Oxford UP, 1984.

Arnett, Jeffrey Jensen. Emerging Adulthood: The Winding Road from the Late Teens through the Twenties. Oxford: Oxford UP, 2004.

---. "The Long and Leisurely Route: Coming of Age in Europe Today." Current History, vol. 106, no. 698, 2007, 130-136.

Austen, Jane. Emma. John Murray, 1816.

Bakhtin, Mikhail M. The Dialogic Imagination: Four Essays. Edited by Michael Holquist, translated by Caryl Emerson and Michael Holquist, U of Texas P, 1981.

---. Problems of Dostojevky's Poetics. Edited by Caryl Emerson. U of Minnesota P, 1984.

Bal, Mieke. Narratology: Introduction to the Theory of Narrative. 3rd ed., translated by Christine van Boheemen, U of Toronto P, 2009.

Batty, Craig. Movies That Move Us: Screenwriting and the Power of the Protagonist's Journey. Palgrave, 2011.

---. "The Physical and Emotional Threads of the Archetypal Hero's Journey: Proposing Common Terminology and Re-examining the Narrative Model." Journal of Screenwriting, vol. 1, no. 2, 2010, pp. 291-308.

Baudrillard, Jean. Impossible Exchange. Translated by Christ Turner, Verso, 2001.

---. Simulacra and Simulation. Translated by Sheila Faria Glaser, U of Michigan P, 1994. Bazin, André. "Adaptation, or the Cinema as Digest." Bazin at Work: Major Essays and Reviews from the Forties and the Fifties, edited by Bert Cardullo, translated by Alain Piette, Routledge, 1997.

Beja, Maurice. Film \& Literature: An Introduction. Longman, 1979. 
Benioff, Daniel, and Daniel Brett Weiss, creators. "Season 8." Game of Thrones, HBO, 2019.

Blatterer, Harry. Coming of Age in Times of Uncertainty. Berghahn, 2009.

---. "The Changing Semantics of Youth and Adulthood." Cultural Sociology, vol. 4. No. 1, 2010, pp. 63-79.

Boon, Kevin Alexander. Script Culture and the American Screenplay. Wayne State UP, 2008.

Booth, Wayne C. The Company We keep: An Ethics of Fiction. U of California P, 1988.

---. The Rhetoric of Fiction. 2nd ed. Penguin, 1983.

Boozer, Jack. "Introduction." Authorship in Film Adaptation, edited by Jack Boozer, U of Texas P, 2008, pp. 1-30.

Bordwell, David. "Neo-structuralist Narratology and the Function of Filmic Storytelling." Narrative Across Media: The Languages of Storytelling, edited by Marie-Laure Ryan, U of Nebraska P, 2004, pp. 203-219.

---. The Way Hollywood Tells It. U of California P, 2006.

Bortolotti, Gary R., and Linda Hutcheon. "On the Origin of Adaptations: Rethinking Fidelity Discourse and 'Success' - biology." New Literary History, vol. 38, no. 3, pp. 443-458.

Bourbon, Brett. Finding a replacement for the soul: Mind and meaning in literature and philosophy. Harvard UP, 2004.

Bowker Market Research. "Young Adult Books Attract Growing Numbers of Adult Fans.” 13 Sept. 2012. Bowker.com, www.bowker.com/news/2012/Young-Adult-Books-Attract-GrowingNumbers-of-Adult-Fans. Accessed 2 Oct. 2017.

Boyd, Brian. On the origin of stories: Evolution, cognition, and fiction. Belknap Press of Harvard UP, 2009.

Brooks, Peter. Reading for the Plot. Knopf, 1984.

Brown, Dan. The Da Vinci Code. Corgi Books, 2003. 
Bryant, John. "Textual Identity and Adaptive Revision: Editing Adaptation as a Fluid Text." Adaptation Studies: New Challenges, New Directions, edited by Jørgen Bruhn et al., Bloomsbury, 2013, pp. 47-68.

Burke, Michael. Literary Reading, Cognition, and Emotion. Routledge, 2011.

Campbell, Joseph. The Hero with a Thousand Faces. 1949. 3rd ed., New World Library, 2008.

Cardwell, Sarah. Adaptation Revisited: Television and the Classic Novel. Manchester UP, 2002.

Carroll, Joseph. Literary Darwinism: Evolution, buman nature, and literature. Routledge, 2004.

---. Reading Human Nature: Literary Darvinism in Theory and Practice. Suny, 2011.

Carroll, Nöel. Minerva's Night Out: Philosophy, Pop Culture, and Moving Pictures. Blackwell, 2013.

---. The Pbilosophy of Motion Pictures. Blackwell, 2008.

Carroll, Rachel. "Introduction: Textual infidelities." Adaptation in Contemporary Culture:

Textual Infidelities, edited by Rachel Carroll, Continuum, 2009, pp 1-10.

Cartmell, Deborah, and Imelda Whelehan. Screen Adaptation: Impure Cinema. Palgrave, 2010.

Cattrysse, Patrick. Descriptive Adaptation Studies: Epistemological and Methodological Issues.

Chatman, Seymour. Story and Discourse: Narrative Structure in Fiction and Film. Cornell UP, 1978.

---. "What Novels Can Do That Films Can't (and Vice Versa)." Critical Inquiry, vol 7, no. 1, 1980, pp. 121-140.

Clarke Arthur C. 2001: A Space Odyssey. Hutchinson, 1968.

---. The Sentinel. BBC, 1948.

Côté, James A. Arrested Adulthood. New York UP, 2000.

Côté, James, and John M. Bynner. "Changes in the transition to adulthood in the UK and Canada: the role of structure and agency in emerging adulthood." Journal of Youth Studies, vol. 11, no. 3, 2008, pp. 251-268. 
Currie, Gregory. Image and Mind: Film, Philosophy and Cognitive Science. Cambridge UP, 1995.

---. Narratives and Narrators: A Philosophy of Stories. Oxford UP, 2010.

Cutchins, Dennis. "Bakhtin, Intertextuality, and Adaptation." The Oxford Handbook of Adaptation Studies, edited by Thomas Leitch, Oxford UP, 2017, pp. 71-86.

Darabont, Frank, director and screenwriter. The Shawshank Redemption. Voice-over performed by Morgan Freeman, Castle Rock Entertainment, 1994.

Derrida, Jacques. Of Grammatology. Translated by Gayatri Chakravorty Spivak, Johns Hopkins UP, 1997.

Diamond, Suzanne. "Whose Life Is It Anyway? Adaptation, Collective Memory, and (Auto)Biographical Processes." Redefining Adaptation Studies, edited by Dennis Cutchins et al., Scarecrow, 2010, pp. 95-110.

Donoghue, Emma. Room. Picador, 2010.

Dutton, Denis. The Art Instinct: Beauty, Pleasure, and Human Evolution. Oxford UP, 2009.

Eco, Umberto. On Literature. Translated by Martin McLaughlin, Mariner Books, 2005.

Eder, Jens, et al. "Characters in Fictional Worlds: An introduction." Characters in Fictional Worlds: Understanding Imaginary Beings in Literature, Film, and Other Media, edited by Jens Eder et al., De Gruyter, 2010, pp. 3-64.

Eder, Jens. "Understanding Characters." Projections, vol. 4, no. 1, 2010, pp. 16-40.

Edmund Husserl, Ideas: General Introduction to Pure Phenomenology. Translated by W. R. Boyce Gibson, Routledge, 2012.

Egri, Lajos. The Art of Dramatic Writing: Its basis in the Creative Interpretation of Human Motives. Touchstone, 2004.

Elliott, Kamilla. "The Adaptation of Adaptation: A Dialogue between the Arts and Sciences." Adaptation and Cultural Appropriation: Literature, Film, and the Arts, edited by Pascal Nicklas and Oliver Lindner, De Gruyter, 2012, pp. 145-161.

---. Retbinking the Novel/Film Debate. Cambridge UP, 2003. 
---. "Theorizing Adaptations/Adapting Theories." Adaptation Studies: New Challenges, New Directions, edited by Jørgen Bruhn et al., Bloomsbury, 2013, pp. 19-46.

Elzinga, Cees, and Aart Liefbroer. "De-standardization of Family-life Trajectories of Young Adults: A Cross-national Comparison Using Sequence Analysis." European Journal of Population, vol. 23, no. 3-4, 2007, pp. 225-250.

Erikson, Erik H. Identity and the life cycle. Norton, 1980.

Falconer, Rachel. The Crossover Novel: Contemporary Children's Fiction and its Adult Readership. Routledge, 2008.

Field, Syd. Screenplay: The Foundations of Screenwriting. Dell, 2005.

Fincher, David, director, and Gillian Flynn, screenwriter. Gone Girl. Twentieth Century Fox, 2014.

Flynn, Gillian. Gone Girl. Phoenix, 2012.

Fokkema, Aleid. Postmodern Characters: A Study of Characterization in British and American Postmodern Fiction. Editions Rodopi, 1991.

Fraser, Nancy. "Rethinking Recognition.” New Left Review, vol.3, no. 3, 2014, pp. 107120.

Genette, Gérard. Narrative Discourse. Cornell UP, 1979.

Geraghty, Christine. "Intertextuality, Contextuality and Textual Analysis in the 'New' Adaptation Studies." Adventures in Textuality: Adaptation Studies in the 21st Century, 3 April, 2013, University of Sunderland, Centre for Research in Media \& Cultural Studies. Keynote Address.

---. Now a Major Motion Picture: Film Adaptations of Literature and Drama. Rowman \& Littlefield, 2008.

Gerrig, Richard. “Concious and Unconcious Processes in Readers' Narrative Experiences." Current Trends in Narratology, edited by Greta Olson, De Gruyter, 2011, pp. 37-61.

Giddens, Anthony. Modernity and Self-Identity: Self and Society in the Late Modern Age. Polity Press, 1991. 
Gillis, Candida. "Multiple Voices, Multiple Genres: Fiction for Young Adults.” The English Journal, vol. 92, no. 2, 2002, pp. 52-59.

Gjelsvik, Anne. "What Novels Can Tell That Movies Can't Show." Adaptation Studies: New Challenges, New Directions, edited by Jørgen Bruhn et. al., Bloomsbury, 2013, pp. $245-264$.

Graehame-Smith, Seth. Pride, Prejudice, and Zombies. Quirk Books, 2009.

Gustin, Jakob. Att göra drama på tematik: Hur Ruben Östlund dramatiserar grupptryck i De ofrivilliga. 2018. Dalarna University, BA thesis.

Habermas, Jürgen. The Philosophical Discourse of Modernity. Translated by Frederick Lawrence, Cambridge University Press, 1987.

Hampton, Christopher. Atonement. Newmarket Press, 2007.

Haneke, Michael, director and screenwriter. Funny Games. Celluloid Dreams, 2007.

Haneke, Michael, director and screenwriter. Funny Games. Wega Film, 1997.

Hendry, Leo, and Marion Kloep. Adolescence and Aduldhood: Transitions and Transformations. Palgrave, 2012.

Hermansson, Joakim. "Adaptations and Post-apocalyptic Atonement." Manuscript submitted for publication, 2020.

---. "Adaptations of Adulthood: Towards a Model for Thematic Rhetoric in Adaptation Studies." New Approaches to Contemporary Adaptation, edited by Betty Kaklamanidou, Wayne State UP, 2020, pp. 173-192.

---. "Characters as Fictional Migrants: Atonement, Adaptation and the Screenplay Process." Journal of Screenwriting vol. 11, no. 1, 2020, pp. 81-97. https://doi.org/10.1386/josc_00014_1.

---. “Okay: The Road and the Good Guys' Adulthood Code." The Cormac McCarthy Journal, vol. 19, no. 1, 2020, forthcoming.

---. 'Strange Masks of Adapted Identities in Patrick McCabe’s Winterwood and The Holy City." Adaptation. apaa011, https:/ / doi-org.www.bibproxy.du.se/ $\underline{10.1093 / a d a p t a t i o n / a p a a 011 . ~}$ 
Hillcoat, John, director, and Joe Penhall, screenwriter. The Road. Dimension Films, 2009.

Hitchcock, Alfred, director, and Joseph Stephano, screenwriter. Psycho. Shamley Productions, 1960.

Hogan, Patrick Colm. Affective Narratology: The Emotional Structure of Stories. Nebraska UP, 2011.

Howard, Ron, director, and Akiva Goldsman, screenwriter. The Da Vinci Code. Columbia, 2006.

Hunter, Ian Q. Cult Film as a Guide to Life: Fandom, Adaptation, and Identity. Bloomsbury, 2017.

Hutcheon, Linda, and Siobhan O'Flynn. A Theory of Adaptation. 2006. 2nd ed., Routledge, 2013.

Igelström, Ann. Narration in the Screenplay Text. 2013. U of Bangor, PhD Dissertation. Iser, Wolfgang. The Act of Reading: A Theory of Aesthetic Response. John Hopkins UP, 1978.

---. The Fictive and the Imaginary: Charting Literary Anthropology. John Hopkins UP, 1993.

James, Erika L. Fifty Shades of Grey. Vintage Books, 2012.

Jameson, Frederic. "Afterword: Adaptation as a Philosophical Problem." True to the Spirit: Film Adaptation and the Question of Fidelity, edited by Colin MacCabe et al., Oxford UP, 2011, pp. 215-235.

Jenkins, Henry. Convergence Culture. New York UP, 2006.

King, Geoff. Indiewood: ... Where Hollywood Meets Independent Cinema. I.B. Tauris, 2009. Kirn, Walter. Up in the Air. Doubleday, 2001.

Konstam, Varda. Emerging and Young Adulthood: Multiple Perspectives, Diverse Narratives. Springer, 2015.

Koss, Melanie D., and William H. Teale. 'What's Happening in YA Literature? Trends in Books for Adolescents." Journal of Adolescent \& Adult Literacy, vol. 52, no. 7,2009 , pp. 563-572. 
Krekula, Clary. "Age coding - on age-based practices of distinction." International Journal of Ageing and Later Life, vol. 4, no 2., 2009, pp. 7-31.

Kubrick, Stanley, director and screenwriter, and Arthur C. Clarke screenwriter. 2001: A Space Odyssey. MGM, 1968.

Kupfer, Joseph H. Visions of Virtue in Popular Film. Westview Press, 1999.

Lehane, Dennis. Shutter Island. Bantam Press, 2003.

Leitch, Thomas. "Against Conclusions: Petit Theories and Adaptation Studies." The Oxford Handbook of Adaptation Studies, edited by Thomas Leitch, Oxford UP, 2017, pp. 698-709.

---. "Introduction." The Oxford Handbook of Adaptation Studies, edited by Thomas Leitch, Oxford UP, 2017, pp. 1-20.

---. "Twelve Fallacies in Contemporary Adaptation Theory." Criticism, vol. 45, no. 2, 2003, pp. 149-171.

---. "What Movies Want." Adaptation Studies: New Challenges, New Directions, edited by Jørgen Bruhn et al., Bloomsbury, 2013, pp. 155-175.

Lindner, Oliver, and Pascal Nicklas. "Adaptation and Cultural Appropriation." Adaptation and Cultural Appropriation: Literature, Film, and the Arts, edited by Pascal Nicklas and Oliver Lindner, De Gruyter, 2012, pp. 1-13.

Lopez-Ropero, Lourdes. "You Are a Flaw in The Pattern': Difference, Autonomy and Bullying in YA Fiction." Children's Literature in Education, vol. 43, no. 2, 2012, pp. 145-157.

Lucas, George, director. Star W ars Episode IV: A New Hope. Twentieth Century Fox, 1977.

Luttrell, Esther. Tools of the Screen Writing Trade. Broadcast Club of America, 1998.

MacCabe, Colin. "Bazinian Adaptation: The Butcher Boy as Example." True to The Spirit: Film Adaptation and the question of Fidelity, edited by Colin MacCabe et al., Oxford UP, 2011, pp. 3-25.

Macdonald, Ian W. Screenwriting Poetics and the Screen Idea. Palgrave, 2013. 
MacKenzie, Ian. "Narratology and Thematics." Modern Fiction Studies, vol. 33, no. 3, Fall 1987, pp. 535-544.

Mackey, Margaret. Narrative Pleasures in Young Adult Novels, Films and Video Games. Palgrave, 2011.

Malick, Terrence, director and screenwriter. The Thin Red Line. Fox 2000 Pictures, 1998.

---. The Tree of Life. Fox Searchlight, 2011.

Maras, Steven. Screenwriting: History, Theory and Practice. Wallflower Press, 2009.

Marcia, James. "Identity and Psychosocial Development in Adulthood." Identity, vol. 2, no. 2, 2002, pp. 7-28.

Margolin, Uri. "The Doer and the Deed: Action as a Basis for Characterization in Narrative." Poetics Today, vol. 7, no. 2, 1986, pp. 205-225.

McCabe Patrick. The Holy City. Bloomsbury, 2008.

---. Winterwood. Bloomsbury, 2006.

McCarey, Leo, director and screenwriter, and Delmer Dawes, screenwriter. Love Affair. RKO, 1939.

---. An Affair to Remember. Jerry Wald Productions, 1957.

McCarthy, Cormac. The Road. Random House, 2007.

McEwan, Ian. Atonement. Jonathan Cape, 2001.

---. Conversations with Ian McEwan. Edited by Ryan Roberts, UP of Mississippi, 2010.

McFarlane, Brian. Novel to Film: An Introduction to the Theory of Adaptation. Oxford UP, 1996.

---. "Reading Film and Literature." The Cambridge Companion to Literature on Screen, edited by Deborah Cartmell and Imelda Whelehan, Cambridge UP, pp. 15-28.

McKee, Robert. Story. Substance, Structure, Style and the Principles of Screenwriting. Methuen, 1999.

Metzinger, Thomas. The Ego Tunnel: The Science of the Mind and the Myth of the Self. Basic Books, 2009.

Millard, Kenneth. Coming of Age in Contemporary American Fiction. Edinburgh UP, 2007. 
Molgat, Marc. "Do Transitions and Social Structures Matter? How 'Emerging Adults' Define Themselves as Adults." Journal of Youth Studies, vol. 10, no. 5, 2007, pp. 495-516.

Moretti, Franco. The Way of the World: The Bildungsroman in European Culture. 2nd ed., Verso, 2000.

Moyes, Jojo. Me Before You. Penguin, 2012.

Murray, Simone. The Adaptation Industry: The Cultural Economy of Contemporary Literary Adaptation. Routledge, 2011.

Nannicelli, Ted. "Why Can't Screenplays Be Artworks?" The Journal of Aesthetics and Art Criticism, vol. 69, no. 4, 2011, pp. 405-414.

Nelms, Beth, Ben Nelms, and Linda Horton. "Young Adult Literature: A Brief but Troubled Season: Problems in YA Fiction." The English Journal, vol. 74, no. 1, 1985, pp. 92-95.

Nikolajeva, Maria. Rhetoric of Character in Children's Literature. Scarecrow Press, 2002.

Nussbaum, Martha C. Love's Knowledge: Essays on Philosphy and Literature. Oxford UP, 1990.

Oatley, Keith. Best Laid Schemes: The Psychology of the Emotions. Cambridge UP, 1992.

---. "Fiction and its study as gateways to the mind." Scientific Study of Literature, vol. 1, no 1, 2011, pp. 153-164.

---. "In the minds of others." Scientific American Mind, vol. 22, no. 5, 2011, pp. $62-67$.

---. Such stuff as dreams: The Psychology of Fiction. Blackwell, 2010.

---. "Theory of mind and theory of minds in literature." Theory of mind and literature, edited by P. Leverage et al., Purdue UP, 2011, pp. 13-26.

Oatley, Keith, and Raymond A. Mar. "Evolutionary pre-adaptation and the idea of character in fiction." Culture and Evolutionary Psychology, vol. 3, 2005, pp. 181196.

---. "The function of fiction is the abstraction and simulation of social experience." Perspectives on Psychological Science, vol. 3, 2008, pp. 173-192. 
Oesterle, Sabrina, et al. "Men's and Women's Pathways to Adulthood and Their Adolescent Precursors." Journal of Marriage and Family, vol. 72, no. 5, 2010, pp. 1436-1453.

“Organism.” Merriam-Webster.com, Merriam-Webster, 2020. Web. 25 April 2020.

Ostry, Elaine. "Is He Still Human? Are You?': Young Adult Science Fiction in the Posthuman Age." The Lion and the Unicorn, vol. 28, no. 2, 2004, pp. 222-246.

Pelican, Kira-Anne, et al. "The Pleistocene protagonist: An evolutionary framework for the analysis of film protagonists." Journal of Screenwriting, vol. 7, no. 3, 2016, pp. 331-349.

Penhall, Joe. The Road. Methuen Drama, 2010.

Phelan, James. Reading People, Reading Plots: Character, Progression, and the Interpretation of Narrative. Chicago: U of Chicago P, 1989.

Price, Steven. A History of the Screenplay. Palgrave, 2013.

---. "Character in the Screenplay Text." Analysing the Screenplay, edited by Jill Nelmes, Routledge, 2011, pp. 201-16.

---. The Screenplay: Authorship, Theory and Criticism. Basingstoke: Palgrave Macmillan, 2010.

Rancière, Jacques. The Politics of Aesthetics. Translated by Gabriel Rockhill, Continuum, 2004.

Raw, Laurence. "Psychology and Adaptation: The Work of Jerome Bruner." Linguaculture, no. 1, 2014, pp. 89-101.

Raymond, Ivan, and Karen Heseltine. "What Does it Mean to be an Adult? Perceptions of Young Men in Residential Care." Child Youth Care Forum, vol. 37, no. 4, 2008, pp. 197-208.

Rayner, Keith. "Eye Movements and Attention in Reading, Scene Perception, and Visual Search.” Quarterly Journal of Experimental Psychology, vol. 62, no. 8, 2009, 1457-1506. 
Reicher, Maria E. “The Ontology of Fictional Characters.” Characters in Fictional Worlds: Understanding Imaginary Beings in Literature, Film, and Other Media, edited by Jens Eder et al., De Gruyter, 2010, pp. 111-133.

Reitman, Ivan, director and screenwriter, and Sheldon Turner, screenwriter. Up in the Air. Paramount, 2010.

Richardson, Brian. "Transtextual Characters." Characters in Fictional Worlds:

Understanding Imaginary Beings in Literature, Film, and Other Media, edited by Jens Eder et al., De Gruyter, 2010, pp. 527-541.

Rimmon-Kenan, Shlomith. Narrative Fiction: Contemporary Poetics. Routledge, 1983.

Rishoi, Christy. From Girl to Woman: American Women's Coming-of-Age Narratives. U of New York P, 2003.

Rushdie, Salman. Imaginary Homelands: Essays and Criticism 1981-1991. Granta Books, 1991.

Ryff, Carol D. "Happiness is everything, or is it?: Explorations on the meaning of psychological well-being." Journal of Personality and Social Psychology, vol. 57, no. 6, 1989, pp. 1069-1081.

Sanders, Julie. Adaptation and Appropriation. Routledge, 2006.

Sant, Gus van, director, and Joseph Stephano, screenwriter. Psycho. Universal Pictures, 1998.

Schober, Regina. "Adaptation as Connection: Transmediality Reconsidered." Adaptation Studies: New Challenges, New Directions, edited by Jørgen Bruhn et al., Bloomsbury, 2013, pp. 89-112.

Scorsese, Martin, director, and Laeta Kalogridis, screenwriter. Shutter Island. Paramount, 2010.

Scott, Ridley, director, and Drew Goddard, screenwriter. The Martian. Twentieth Century Fox, 2015.

Sharrock, Thea, director, and Jojo Moyes, screenwriter. Me Before You. MGM, 2016. Shary, Timothy. Generation Multuplex: The Image of Youth in American Cinema since 1960. U of Texas P, 2014. 
Shurlock, Geoffrey. "The Motion Picture Production Code." The Annals of the American Academy of Political and Social Science, vol. 254, 1947, pp. 140-146.

Smith, Murray. Engaging Characters: Fiction, Emotion, and the Cinema. Clarendon Press, 1995.

Stam, Robert. "Introduction: The Theory and Practice of Adaptation." Literature and Film: A Guide to the Theory and Practice of Film Adaptation, edited by Robert Stam and Alessandra Raengo, Blackwell, 2005, pp. 1-52.

Stapele, Peter van. Poetics of the Screenplay as Drama-Text. 2005. Gerard Tuning Institute, $\mathrm{PhD}$ Dissertation.

Steers, Burr, director and screenwriter. Pride, Prejudice, and Zombies. Cross Creek Pictures, 2016.

Sternberg, Claudia. Written for the Screen: The American Motion-Picture Screenplay as Text. Stauffenburg, 1997.

Stewart, Susan. "In The Ellison Tradition: In/Visible Bodies of Adolescent and YA Fiction." Children's Literature in Education, vol. 40, no. 3, 2009, pp. 180-196.

Taylor-Wood, Sam, director, and Kelly Marcel, screenwriter. Fifty Shades of Grey. Focus Features, 2015.

Thompson, Kristin. Storytelling in the New Hollywood: Understanding Classical Narrative Technique. Harvard UP, 1999.

Ting-Toomey, Stella, and Leeva C. Chung. Understanding intercultural communication. Oxford UP, 2012.

Tomashevsky, Boris. "Thematics." Russian Formalist Criticism: Four Essays, edited and translated by Lee T. Lemon and Marion J. Ries, Bison Books, 1965, pp.61-95. Truby, John. The Anatomy of Story: 22 Steps to Becoming a Master Storyteller. Farrar, Straus and Giroux, 2007.

Ulmer, Greg. Teletheory. Atropos Press, 2004.

Vogler, Christopher. The Writer's Journey: Mythic Structure for Writers. $3^{\text {rd }}$ ed., Michael Wiese Productions, 2007.

Wagner, Geoffrey. The Novel and the Cinema. Tantity, 1975. 
Walton, Kendall. Mimesis as Make-Believe: On the Foundations of the Representational Arts. Harvard UP, 1990.

---. "Reply to Reviewers." Philosopby and Phenomenological Research, vol. 51, no. 2, 1991, pp. 413-431.

Ward, Coleen, et al. The Psychology of Culture Shock. Routledge, 2005.

Weir, Andy. The Martian. Random House, 2014.

Whelehan, Imelda. "Adaptations: The Contemporary Dilemmas." Adaptations: From Text to Screen, Screen to Text, edited by Deborah Cartmell and Imelda Whelehan, Routledge, 1999, pp. 3-20.

Wiegand, David. "Stumbling into Fate: Accidents and Choices Trip Up the Characters in Ian McEwan's New Novel." Review of Atonement by Ian McEwan, San Francisco Gate, 10 Mar. 2002, https://www.sfgate.com/books/article/Stumbling-into-fate-Accidents-andchoices-trip-2866997.php. Accessed 5 Sep. 2013.

Wohlmann, Anita. Aged Young Adults: Age Readings of Contemporary American Novels and Films. Transcript, 2014.

Wright, Joe, director, and Christopher Hampton, screenwriter Atonement. Universal Pictures, 2007.

Zukauskiene, Rita, editor. Emerging Adulthood in a European Context. Routledge, 2015. Zunshine, Lisa. Why We Read Fiction: Theory of Mind and the Novel. Ohio State U P, 2006.

Östlund, Ruben, director and screenwriter, and Erik Hemmendorff, screenwriter. Involuntary. Plattform Produktion, 2008. 
Adapting Adulthood 


\section{Appendices}

\section{Articles A-E}

\section{Article A}

Joakim Hermansson. "Strange Masks of Adapted Identities in Patrick McCabe's

Winterwood and The Holy City." Adaptation. apaa011, https://doi-

org.www.bibproxy.du.se/ 10.1093/adaptation/apaa011.

\section{Article B}

Joakim Hermansson. "Characters as Fictional Migrants: Atonement, Adaptation and the Screenplay Process." Journal of Screenwriting vol. 11, no. 1, 2020, pp. 81-97. https://doi.org/10.1386/josc_00014_1.

\section{Article C}

Joakim Hermansson. "Adaptations of Adulthood: Towards a Model for Thematic Rhetoric in Adaptation Studies." New Approaches to Contemporary Adaptation, edited by Betty Kaklamanidou, Wayne State UP, 2020, pp. 173-192.

\section{Article D}

Joakim Hermansson. "Okay: The Road and the Good Guys' Adulthood Code." The Cormac McCarthy Journal, vol. 19, no. 1, 2020, forthcoming.

\section{Article E}

Joakim Hermansson. "Adaptations and Post-apocalyptic Atonement." Manuscript submitted for publication, 2020. 



\section{Article A}

Strange Masks of Adapted Identities in Patrick McCabe's

Winterwood and The Holy City 



\section{Article B}

Characters as Fictional Migrants: Atonement, Adaptation and the Screenplay Process 



\section{Article C}

Adaptations of Adulthood: Towards a Model for Thematic

Rhetoric in Adaptation Studies 



\section{Article D}

Okay: The Road and the Good Guys' Adulthood Code 



\section{Article E}

Adaptations and Post-apocalyptic Atonement 




\section{Adapting Adul thood}

\section{Migrating Characters and Themes \\ from Novels, screenplays, and Films}

When novels are adapted for the screen, the fietional characters are inevitably trans formed in the adaptation process, and so is the thematic content. This study considers the characters and the thematic argument of a s tory as migrants who leave the land of the novel in order to adapt to a life on the screen with transformed self identities:

In the five articles that this thesis is based upon, various sociological perspectives are engaged, focusing on what happens to the representation of adulthood when novels are adapted for the screen. They explore popular works of fiction Hike Atonement Fify Shades of Cryy, Gone Ch, Me before You, Room, Shuter Tland, The Da Vinci Code. The Marian, The Road Up in the Ath and novels by Patrick McCabe.

Because nove -screenplay-film adaptations comprise alternative versions of a story, with their complementary thematic arguments, they constitute particularly rich thematic representations and metaphors for what social adaptation requires of us. Also, with an adaptation-minded approach, novelescreenplay film adaptations may be regarded as processes and objects at the same time, with each work of fiction as an integral part of a greater dynamic whole:

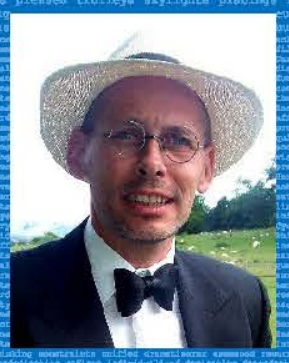

Joakim $/$ ermansson teaches pedagogy, screenwriting; adaptation and film theory at Dalarna University. De is a doctoral candidate-at University of Gothenburg and Thas published on screenwriting and adaptation theory.

ISB N: 978-91-8009-198-5 (PRINT)

ISBN: $978-91-8009-199=2$ (PDI) 